\title{
Critical Rotational Speeds for Superfluids in Homogeneous Traps
}

\author{
M. Correggi ${ }^{a}$, F. Pinsker ${ }^{b}$, N. Rougerie ${ }^{c}$, J. Yngvason $^{d, e}$ \\ ${ }^{a}$ Dipartimento di Matematica, Università degli Studi Roma Tre, \\ L.go S. Leonardo Murialdo 1, 00146, Rome, Italy. \\ ${ }^{b}$ DAMTP, University of Cambridge, Wilbertforce Road, Cambridge CB3 0WA, United Kingdom. \\ ${ }^{c}$ Université Grenoble 1 and CNRS, LPMMC, UMR 5493, BP 166, 38042 Grenoble, France. \\ ${ }^{d}$ Fakultät für Physik, Universität Wien, Boltzmanngasse 5, 1090 Vienna, Austria. \\ e Erwin Schrödinger Institute for Mathematical Physics, Boltzmanngasse 9, 1090 Vienna, Austria.
}

September 8, 2018

Dedicated to Elliott H. Lieb on the occasion of his 80th birthday

\begin{abstract}
We present an asymptotic analysis of the effects of rapid rotation on the ground state properties of a superfluid confined in a two-dimensional trap. The trapping potential is assumed to be radial and homogeneous of degree larger than two in addition to a quadratic term. Three critical rotational velocities are identified, marking respectively the first appearance of vortices, the creation of a 'hole' of low density within a vortex lattice, and the emergence of a giant vortex state free of vortices in the bulk. These phenomena have previously been established rigorously for a 'flat' trap with fixed boundary but the 'soft' traps considered in the present paper exhibit some significant differences, in particular the giant vortex regime, that necessitate a new approach. These differences concern both the shape of the bulk profile and the size of vortices relative to the width of the annulus where the bulk of the superfluid resides. Close to the giant vortex transition the profile is of Thomas-Fermi type in 'flat' traps, whereas it is gaussian for soft traps, and the 'last' vortices to survive in the bulk before the giant vortex transition are small relative to the width of the annulus in the former case but of comparable size in the latter.
\end{abstract}

MSC: 35Q55,47J30,76M23. PACS: 03.75.Hh, 47.32.-y, 47.37.+q.

Keywords: Bose-Einstein Condensates, Superfluidity, Vortices, Giant Vortex.

\section{Contents}

1 Introduction and Main Results 
2 The Regime $|\log \varepsilon| \ll \omega, \Omega \ll \varepsilon^{-4} \quad 15$

2.1 The TF Energies and Densities . . . . . . . . . . . . . . . . . . . . . . . . . . . . . .

2.2 Energy Asymptotics and Emergence of a Hole . . . . . . . . . . . . . . . . . . . . . . . . 19

2.3 Uniform Distribution of Vorticity . . . . . . . . . . . . . . . . . . . . 24

3 The Giant Vortex Regime $\Omega \sim \varepsilon^{-4} \quad 25$

3.1 Preliminary Estimates for $E^{\mathrm{GP}}$ and $\psi^{\mathrm{GP}} \ldots \ldots \ldots \ldots \ldots \ldots \ldots \ldots$

3.2 The Giant Vortex Density Profile . . . . . . . . . . . . . . . . . . . . . . . . . . . 28

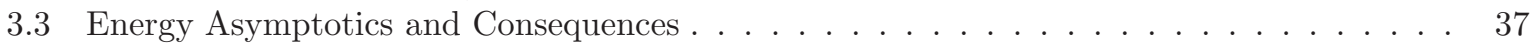

3.4 Transition to the Giant Vortex State . . . . . . . . . . . . . . . . . . . . . . 43

\section{Rotational Symmetry Breaking}

\section{Introduction and Main Results}

Since their first experimental realization in 1995, atomic Bose-Einstein condensates (BECs) have become a subject of tremendous interest, stimulating intense theoretical activity. The BE-condensed alkali vapors that are nowadays produced in many laboratories offer a spectacular level of tunability for several experimental parameters, making them a favorite testing ground for many intriguing quantum phenomena. Among these is superfluidity, i.e., the occurrence of frictionless flow, previously observed in liquid helium. Atomic BECs offer a valuable alternative to the latter system, since experiments with dilute gases of ultracold atoms allow to test theories of superfluidity in much more detail.

From a theoretical point of view an appealing aspect of the field is its sound mathematical foundation. The most commonly used model for the description of BECs, the so-called Gross-Pitaevskii (GP) theory, is now supported both by extensive comparisons with experiments [A, Fe1 and a solid mathematical basis LSSY]. Indeed, substantial advances on the connection between GP theory and many-body quantum physics have been made in recent years, leading to a rigorous derivation of both the stationary LSY1, LiS, BCPY] and dynamic aspects [ESY1, ESY2, [P] of the theory.

One of the most striking features of superfluids is their response to rotation of the confining trap. Typical experiments (see [Fe1] for further references) start by cooling a Bose gas in a magneto-optical trap below the critical temperature for Bose-Einstein condensation. If the trap is set in rotational motion the gas stays to begin with at rest in the inertial frame, but if the rotation speed exceeds some critical value (called $\Omega_{\mathrm{c}_{1}}$ below), vortices are nucleated and remain stable over a long time span. This behavior is in strong contrast with that of a classical fluid, which in the stationary state rotates like a rigid body and thus remains at rest in the rotating frame.

In a rotationally symmetric trap, the main two experimentally tunable parameters are the rotation speed $\Omega_{\text {rot }}$ and the strength of interparticle interactions, written as $1 / \varepsilon^{2}$ with $\varepsilon>0$ in the sequel. The ground state of the system strongly depends on the relation between these two parameters.

In this paper we study the ground state of a rotating Bose gas in the framework of the two-dimensional GP theory 1. We consider the minimization, under a unit mass constraint, of the GP functiona 2

$$
\mathcal{E}_{\text {phys }}^{\mathrm{GP}}[\Psi]=\int_{\mathbb{R}^{2}} \mathrm{~d} \mathbf{r}\left\{\frac{1}{2}\left|\left(\nabla-i \mathbf{A}_{\text {rot }}\right) \Psi\right|^{2}+\left(V(r)-\frac{1}{2} \Omega_{\text {rot }}^{2} r^{2}\right)|\Psi|^{2}+\frac{|\Psi|^{4}}{\varepsilon^{2}}\right\}
$$

with

$$
\mathbf{A}_{\text {rot }}:=\Omega_{\text {rot }} \wedge \mathbf{r}=\Omega_{\text {rot }} r \mathbf{e}_{\vartheta},
$$

\footnotetext{
${ }^{1}$ Such a description is justified if the trap almost confines the gas on a plain orthogonal to the axis of rotation or, on the contrary, the trap is very elongated along the axis, in which case the behavior can be expected to be essentially independent of the coordinate in that direction.

${ }^{2}$ The subscript 'phys' stands for physical, i.e., this is the functional in the original physical variables, as opposed to the rescaled functionals introduced in Sect. 1.1 below. Units have been chosen such that $\bar{h}$ as well as the mass are equal to 1 .
} 
where $\boldsymbol{\Omega}_{\text {rot }}$ is the rotational velocity, $r=|\mathbf{r}|$ with $\mathbf{r} \in \mathbb{R}^{2}$ is the distance from the rotation axis, and $\mathbf{e}_{\vartheta}$ stands for the unit vector in the direction transverse to $\mathbf{r}$. The confinement is provided by a potential of the form

$$
V(r):=k r^{s}+\frac{1}{2} \Omega_{\mathrm{osc}}^{2} r^{2}
$$

with $k>0$. We restrict to the case $2<s<\infty$ (anharmonic, 'soft' potentials) and shall mainly study the Thomas-Fermi (TF) limit $\varepsilon \rightarrow 0$. The case $s=2$ is special in many respects because the potential $-\Omega_{\text {rot }}^{2} r^{2}$ due to the centrifugal force is also a quadratic function of $r$. As a consequence an upper bound is set to the allowed values of the rotational speed and different physics is expected in the regime where the centrifugal force nearly compensates the trapping force (see [A] and references therein, LSY2] and LeS].

We have studied before CDY1, CRY, CPRY1, R2 the case of a flat trap with the unit disc as boundary. It corresponds formally to the potential

$$
V(r)= \begin{cases}0, & \text { if } r \leq 1 \\ +\infty, & \text { if } r>1\end{cases}
$$

which amounts to restricting the integration domain to the unit disc. This model can formally be regarded as the $s \rightarrow+\infty$ limit of the theory with the trap (1.3) but we emphasize that this statement has to be taken with care. Indeed, as revealed by the analysis of the present paper (see also CPRY2, Sect. III) the limit $s \rightarrow+\infty$ cannot be interchanged with the TF limit $\varepsilon \rightarrow 0$, which is the basis of most rigorous treatments of the GP theory, including the present one.

Provided the confining potential is stronger than quadratic, i.e., $s>2$, one can distinguish three critical speeds at which major phase transitions occur in the BEC (references in the literature concerning these phenomena include [CD, Fe2, FB, FJS, FZ, KTU, KB, KF]):

- When $\Omega_{\text {rot }}<\Omega_{\mathrm{c}_{1}}$ the condensate is vortex-free. Vortices start to appear when the rotation speed exceeds $\Omega_{\mathrm{C}_{1}}$ and form regular patterns. An adaptation of the methods of AAB, IM1, IM2, AJR, shows that $\Omega_{c_{1}} \propto \varepsilon^{4 /(s+2)}|\log \varepsilon|$. This is valid for all traps, including the harmonic trap $s=2$ and the flat trap, since the centrifugal force is negligible in this regime.

- When $\Omega_{\mathrm{c}_{1}} \ll \Omega_{\text {rot }} \ll \Omega_{\mathrm{c}_{2}}$ vortices are densely packed and uniformly distributed in the condensate. It is observed both numerically and experimentally that they arrange themselves into triangular arrays (Abrikosov lattices with hexagonal unit cells). It remains an open problem to provide a rigorous proof of this fact but a heuristic argument based on an electrostatic analogy and Newton's Theorem can be found in $\mathrm{CY}$ ] and SS3 contains a rigorous proof for a simplified model. The distribution of vorticity has been shown to be uniform in this regime for the flat trap case both with Neumann [CY and Dirichlet boundary conditions CPRY1. In this paper we adapt this result to the case of soft potentials.

- When $\Omega_{\text {rot }}$ reaches the second critical speed $\Omega_{\mathrm{c}_{2}}$, the centrifugal force dips a hole with strongly depleted matter density around the center of the trap. Vortices are however still uniformly distributed in the annular bulk where the mass of the condensate resides. In the flat trap case the second critical speed is of order $\varepsilon^{-1}$ [CDY1, CRY, CPRY1. As shown in CDY2 and discussed further in Section 1.2 below, this order is changed in the case of a soft potential (1.3) to $\varepsilon^{\frac{4}{s+2}-1}$, provided $s>2$. Notice that in the limit $s \rightarrow \infty$ we recover the result for the flat trap case, despite the subtlety of the exchange of limits (see below). Here the behavior of the gas differs markedly from that in a purely harmonic trap where centrifugal effects start to compensate the trapping potential in the regime $\Omega_{\text {rot }}=\mathcal{O}(1)$. In this case the state of the condensate is well approximated

\footnotetext{
${ }^{3}$ Note that in the cited papers the GP functional is rescaled from the outset, as in Eq. (1.12) below, and in the rescaled variables the critical velocity is $\omega_{c 1} \propto|\log \varepsilon|$.
} 
by a wave function in the lowest Landau level of the magnetic Hamiltonian corresponding to the first term in (1.1) $\mathrm{ABN}$, AB, LSY2] and the GP theory ultimately loses its validity [LeS].

- When $\Omega_{\text {rot }}$ is further increased above a third critical speed $\Omega_{\mathrm{c}_{3}}$, a new phase transition occurs where vortices are expelled from the bulk of the condensate. All the vorticity resides in the central hole created by the centrifugal force, resulting in a giant vortex phase. One of the main results of this paper is that, for a potential of the form (1.3) with $s>2, \Omega_{\mathrm{c}_{3}}$ is of order $\varepsilon^{-4 \frac{s-2}{s+2}}$. This converges to $\varepsilon^{-4}$ when $s \rightarrow+\infty$, which is significantly larger than the value of $\Omega_{\mathrm{c}_{3}}$ in the flat trap case. The latter is, to leading order in $\varepsilon$, given by $(2 /(3 \pi))\left(\varepsilon^{2}|\log \varepsilon|\right)^{-1}$ CRY] R2. The limits $\varepsilon \rightarrow 0$ and $s \rightarrow+\infty$ can thus not be interchanged in this regime. As we explain further below, this is due to significantly different physical properties of the condensate in the two kinds of traps close to the transition regime.

- When $\Omega_{\text {rot }} \gg \Omega_{\mathrm{c}_{3}}$, the BEC is in a giant vortex phase and the energy is given to an excellent approximation by a trial function with a single multiple vortex at the origin (of degree $\Omega_{\text {rot }}$ to leading approximation), accounting for the macroscopic circulation. Nevertheless we prove that rotational symmetry is still broken in any true ground state, namely that no ground state can be an eigenfunction of angular momentum.

The TF approximation in the regime $\Omega_{\mathrm{c}_{1}} \ll \Omega_{\text {rot }} \ll \Omega_{\mathrm{c}_{3}}$ was studied in CDY2 where a preliminary upper bound for the vortex contribution was given. Precise upper and lower bounds are provided in Theorem 1.1 below. The case of large rotation speeds with fixed coupling constant $\varepsilon^{-2}$ has been considered before [R1] for the special case of a quartic potential (i.e., $s=4$ ). The present contribution improves the results of that paper in several directions. In particular, although the appearance of a giant vortex state has been proved in [R1, no rigorous estimate of the third critical speed could be provided. Such an estimate is given in Theorem 1.3 below.

In the remaining subsections of this introduction we define our setting more precisely and state our main results. We start by discussing the scalings we shall use to study the functional (1.1). We then state our results about the regimes $\Omega_{\mathrm{c}_{1}} \ll \Omega_{\text {rot }} \ll \Omega_{\mathrm{c}_{3}}$ and $\Omega_{\mathrm{rot}} \propto \Omega_{\mathrm{c}_{3}}$ in two different subsections and finally present our symmetry breaking theorem.

For a concise discussion of our results and methods the reader is referred to the companion paper CPRY2.

\subsection{Scalings of the GP Functional}

In this paper we focus on the analysis of the minimization of the GP energy functional (1.1) under the mass constraint

$$
\|\Psi\|_{2}^{2}:=\int_{\mathbb{R}^{2}} \mathrm{~d} \mathbf{r}|\Psi|^{2}=1
$$

The trapping is given by the potential (1.3) with $\Omega_{\mathrm{osc}}<\Omega_{\mathrm{rot}}$, and $0<\varepsilon \ll 1$, i.e., we study the TF limit of the model. The power $s$ in (1.3) characterizes the homogeneous trap and we assume that $2<s<\infty$. We also introduce the parameter

$$
\Omega_{\mathrm{eff}}:=\sqrt{\Omega_{\mathrm{rot}}^{2}-\Omega_{\mathrm{osc}}^{2}},
$$

so that the effective potential in (1.1) can be written in the form

$$
V(r)-\frac{1}{2} \Omega_{\mathrm{rot}}^{2} r^{2}=k r^{s}-\frac{1}{2} \Omega_{\mathrm{eff}} r^{2} .
$$

Since we are interested in exploring the rotation regime $\Omega_{\text {rot }} \rightarrow \infty$ but want also to keep track of the effect of the quadratic term in the expression above we shall assume that

$$
\Omega_{\mathrm{eff}}^{2}=\gamma \Omega_{\mathrm{rot}}^{2}
$$


for some given $0<\gamma \leq 1$.

Since the profile of a minimizer of (1.1) expands if $\Omega_{\text {rot }} \rightarrow \infty$ and/or $\varepsilon \rightarrow 0$ it is natural to rescale all lengths as well as the rotational velocity. In fact it is convenient to employ two different scalings in different asymptotic regimes: If

$$
\Omega_{\text {rot }} \lesssim \varepsilon^{-\frac{s-2}{s+2}}
$$

we define $\mathbf{x} \in \mathbb{R}^{2}, x=|\mathbf{x}|, \psi(\mathbf{x}), \omega$ and $\mathbf{A}_{\omega}$ by writing

$$
\mathbf{r}=R_{\varepsilon} \mathbf{x}, \quad \Psi(\mathbf{r})=R_{\varepsilon}^{-1} \psi(\mathbf{x}), \quad \Omega_{\mathrm{rot}}=R_{\varepsilon}^{-2} \omega, \quad \mathbf{A}_{\omega}=\omega x \mathbf{e}_{\vartheta},
$$

where

$$
R_{\varepsilon}:=\left(k \varepsilon^{2}\right)^{-\frac{1}{s+2}}=\mathcal{O}\left(\varepsilon^{-\frac{2}{s+2}}\right) .
$$

With these scalings the functional (1.1) can be rewritten as

$$
\mathcal{E}_{\text {phys }}^{\mathrm{GP}}[\Psi]=R_{\varepsilon}^{-2} \mathcal{E}_{\omega}^{\mathrm{GP}}[\psi],
$$

with

$$
\mathcal{E}_{\omega}^{\mathrm{GP}}[\psi]:=\int_{\mathbb{R}^{2}} \mathrm{~d} \mathbf{x}\left\{\frac{1}{2}\left|\left(\nabla-i \mathbf{A}_{\omega}\right) \psi\right|^{2}+\varepsilon^{-2}\left[x^{s}|\psi|^{2}-\frac{1}{2} \gamma \varepsilon^{2} \omega^{2} x^{2}|\psi|^{2}+|\psi|^{4}\right]\right\} .
$$

If on the other hand

$$
\Omega_{\mathrm{rot}} \gtrsim \varepsilon^{-\frac{s-2}{s+2}},
$$

a natural scaling parameter is given by the position $R_{\mathrm{m}}$ of the unique minimum point of the effective potential (1.6), which is explicitly given by

$$
R_{\mathrm{m}}:=\left(\frac{\gamma \Omega_{\mathrm{rot}}^{2}}{s k}\right)^{\frac{1}{s-2}}=\mathcal{O}\left(\Omega_{\mathrm{rot}}^{\frac{2}{s-2}}\right)
$$

Writing now

$$
\mathbf{r}=R_{\mathrm{m}} \mathbf{x}, \quad \Psi(\mathbf{r})=R_{\mathrm{m}}^{-1} \psi(\mathbf{x}), \quad \Omega_{\mathrm{rot}}=R_{\mathrm{m}}^{-2} \Omega, \quad \mathbf{A}_{\Omega}=\Omega x \mathbf{e}_{\vartheta},
$$

the GP energy functional (1.1) becomes

$$
\mathcal{E}_{\text {phys }}^{\mathrm{GP}}[\Psi]=R_{\mathrm{m}}^{-2}\left[\mathcal{E}_{\Omega}^{\mathrm{GP}}[\psi]+\left(\frac{1}{s}-\frac{1}{2}\right) \gamma \Omega^{2}\right],
$$

with

$$
\mathcal{E}_{\Omega}^{\mathrm{GP}}[\psi]:=\int_{\mathbb{R}^{2}} \mathrm{~d} \mathbf{x}\left\{\frac{1}{2}\left|\left(\nabla-i \mathbf{A}_{\Omega}\right) \psi\right|^{2}+\gamma \Omega^{2} W(x)|\psi|^{2}+\varepsilon^{-2}|\psi|^{4}\right\},
$$

where the effective potential is given by

$$
W(x):=\frac{x^{s}-1}{s}-\frac{x^{2}-1}{2} .
$$

Note that for convenience we have extracted a negative additive constant in (1.11) exploiting the $L^{2}$ normalization of both $\Psi$ and $\psi$, in order to obtain a positive trapping potential $W \geq 0$ with

$$
\inf _{x \in \mathbb{R}^{+}} W(x)=W(1)=0 .
$$

Note also that

$$
\Omega_{\mathrm{rot}}=\left(k \varepsilon^{2}\right)^{\frac{2}{s+2}} \omega=(s k / \gamma)^{\frac{2}{s+2}} \Omega^{\frac{s-2}{s+2}}
$$


and the two rescaled velocities, $\omega$ and $\Omega$, are related by

$$
\omega=(s / \gamma)^{\frac{2}{s+2}} \varepsilon^{-\frac{4}{s+2}} \Omega^{\frac{s-2}{s+2}} .
$$

For $s \rightarrow \infty$, i.e., a flat trap, $\omega=\Omega=\Omega_{\text {rot }}$.

To find the ground state energy and wave function of the system, we minimize the functionals (1.12) or (1.17) on the domain

$$
\mathscr{D}^{\mathrm{GP}}:=\left\{\psi \in H^{1}\left(\mathbb{R}^{2}\right):\|\psi\|_{2}=1\right\} .
$$

We introduce the notation

$$
E_{\omega}^{\mathrm{GP}}:=\inf _{\psi \in \mathscr{D} \mathrm{GP}} \mathcal{E}_{\omega}^{\mathrm{GP}}[\psi], \quad E_{\Omega}^{\mathrm{GP}}:=\inf _{\psi \in \mathscr{D} \mathrm{GP}} \mathcal{E}_{\Omega}^{\mathrm{GP}}[\psi],
$$

for the ground state energies. Any corresponding minimizer will be denoted by $\psi_{\omega}^{\mathrm{GP}}$ or $\psi_{\Omega}^{\mathrm{GP}}$ respectively. The GP minimizer is in general not unique because vortices can break the rotational symmetry (see Section 1.4) but any minimizer satisfies in $\mathbb{R}^{2}$ a variational equation (GP equation) which is in the case of (1.12)

$$
-\frac{1}{2} \Delta \psi_{\omega}^{\mathrm{GP}}-\omega L_{z} \psi_{\omega}^{\mathrm{GP}}+\varepsilon^{-2}\left[r^{s}+\frac{1}{2}(1-\gamma) \varepsilon^{2} \omega^{2} x^{2}+2\left|\psi_{\omega}^{\mathrm{GP}}\right|^{2}\right] \psi_{\omega}^{\mathrm{GP}}=\mu_{\omega}^{\mathrm{GP}} \psi_{\omega}^{\mathrm{GP}},
$$

whereas for (1.17) it becomes

$$
-\frac{1}{2} \Delta \psi_{\Omega}^{\mathrm{GP}}-\Omega L_{z} \psi_{\Omega}^{\mathrm{GP}}+\frac{1}{2} \Omega^{2} x^{2} \psi_{\Omega}^{\mathrm{GP}}+\gamma \Omega^{2} W(x) \psi_{\Omega}^{\mathrm{GP}}+2 \varepsilon^{-2}\left|\psi_{\Omega}^{\mathrm{GP}}\right|^{2} \psi_{\Omega}^{\mathrm{GP}}=\mu_{\Omega}^{\mathrm{GP}} \psi_{\Omega}^{\mathrm{GP}} .
$$

Here $L_{z}=-i \partial_{\vartheta}$ stands for the $z$-component of the angular momentum and the chemical potentials $\mu_{\omega}^{\mathrm{GP}}$ and $\mu_{\Omega}^{\mathrm{GP}}$ are fixed by imposing the $L^{2}$-normalization condition on $\psi_{\omega}^{\mathrm{GP}}$ and $\psi_{\Omega}^{\mathrm{GP}}$ respectively and are given by

$$
\mu_{\omega}^{\mathrm{GP}}=E_{\omega}^{\mathrm{GP}}+\varepsilon^{-2} \int_{\mathbb{R}^{2}} \mathrm{~d} \mathbf{x}\left|\psi_{\omega}^{\mathrm{GP}}\right|^{4}, \quad \mu_{\Omega}^{\mathrm{GP}}=E_{\Omega}^{\mathrm{GP}}+\varepsilon^{-2} \int_{\mathbb{R}^{2}} \mathrm{~d} \mathbf{x}\left|\psi_{\Omega}^{\mathrm{GP}}\right|^{4} .
$$

The functional (1.12) is used to analyze the behavior of the superfluid when $\Omega_{\mathrm{c}_{1}} \ll \Omega_{\mathrm{rot}} \ll \Omega_{\mathrm{c}_{2}}$, which corresponds to $|\log \varepsilon| \ll \omega \ll \varepsilon^{-1}$ whereas the scaling leading to (1.17) is more suited to the regime $\Omega_{\text {rot }} \gg \Omega_{\mathrm{c}_{2}}$, corresponding to $\varepsilon^{-1} \ll \Omega$. The transition to a giant vortex phase occurs when $\Omega \propto \varepsilon^{-4}$.

For the sake of simplicity we will often omit the suffix $\omega$ or $\Omega$ in $\psi_{\omega}^{\mathrm{GP}}, \psi_{\Omega}^{\mathrm{GP}}, E_{\omega}^{\mathrm{GP}}$, etc., when it is clear the regime under discussion and/or the statement applies to all the regimes taken into account in the paper, i.e., $|\log \varepsilon| \ll \omega, \Omega \lesssim \varepsilon^{-4}$.

\subsection{Between $\Omega_{\mathrm{c}_{1}}$ and $\Omega_{\mathrm{c}_{3}}$ : Vortex Lattice and Emergence of a Hole}

As we are going to see the leading order contribution to the GP energy $E^{\mathrm{GP}}$ is determined in this regime by the density $\rho^{\mathrm{GP}}:=\left|\psi^{\mathrm{GP}}\right|^{2}$, not the phase of the order parameter. Correspondingly we introduce a TF-like functional acting only on a density $\rho$ : If

$$
|\log \varepsilon| \ll \omega \lesssim \varepsilon^{-1},
$$

we set

$$
\mathcal{E}_{\omega}^{\mathrm{TF}}[\rho]=\varepsilon^{-2} \int_{\mathbb{R}^{2}} \mathrm{~d} \mathbf{x}\left[\left(x^{s}+\rho\right) \rho-\frac{1}{2} \gamma \varepsilon^{2} \omega^{2} x^{2} \rho\right],
$$

whith $\rho$ belonging to the domain

$$
\mathscr{D}^{\mathrm{TF}}:=\left\{\rho \in L^{2}\left(\mathbb{R}^{2}\right),\|\rho\|_{1}=1: \rho \geq 0, x^{s} \rho \in L^{1}\left(\mathbb{R}^{2}\right)\right\} .
$$


The minimization of $\mathcal{E}^{\mathrm{TF}}$ is straightforward (see Section 2.1) and both the energy and minimizer have explicit expressions: The latter is the compactly supported function

$$
\rho_{\omega}^{\mathrm{TF}}(x)=\frac{1}{2}\left[\varepsilon^{2} \mu_{\omega}^{\mathrm{TF}}-x^{s}+\frac{1}{2} \gamma \varepsilon^{2} \omega^{2} x^{2}\right]_{+},
$$

where $\mu_{\omega}^{\mathrm{TF}}$ is determined by the $L^{1}$-normalization and $[\cdot]_{+}$stands for the positive part. We also set

$$
E_{\omega}^{\mathrm{TF}}:=\inf _{\rho \in \mathscr{D}^{\mathrm{TF}}} \mathcal{E}_{\omega}^{\mathrm{TF}}[\rho]=\mathcal{E}_{\omega}^{\mathrm{TF}}\left[\rho_{\omega}^{\mathrm{TF}}\right]
$$

On the other hand if

$$
\varepsilon^{-1} \lesssim \Omega \ll \varepsilon^{-4}
$$

the scaling defined in (1.15) becomes more useful and it is convenient to define the TF functional as

$$
\mathcal{E}_{\Omega}^{\mathrm{TF}}[\rho]=\int_{\mathbb{R}^{2}} \mathrm{~d} \mathbf{x}\left[\varepsilon^{-2} \rho^{2}+\gamma \Omega^{2} W(x) \rho\right]
$$

with $\rho \in \mathscr{D}^{\mathrm{TF}}$. The TF energy and minimizer, defined analogously to (1.31), are now denoted by $E_{\Omega}^{\mathrm{TF}}$ and $\rho_{\Omega}^{\mathrm{TF}}$ respectively. The explicit expression for the latter is

$$
\rho_{\Omega}^{\mathrm{TF}}(x)=\frac{1}{2}\left[\varepsilon^{2} \mu_{\Omega}^{\mathrm{TF}}-\gamma \varepsilon^{2} \Omega^{2} W(x)\right]_{+} .
$$

As we are going to see (see Section 2.1 for further details) there are critical values $\omega_{\mathrm{c}}, \Omega_{\mathrm{c}}=\mathcal{O}\left(\varepsilon^{-1}\right)$ of the angular velocity satisfying

$$
\Omega_{\mathrm{c}}=R_{\mathrm{m}}^{2} R_{\varepsilon}^{-2} \omega_{\mathrm{c}}
$$

such that if $\omega>\omega_{\mathrm{c}}$ (resp. $\Omega>\Omega_{\mathrm{c}}$ ) then the TF density $\rho_{\omega}^{\mathrm{TF}}$ (resp. $\rho_{\Omega}^{\mathrm{TF}}$ ) vanishes at the origin. More precisely, if $\Omega>\Omega_{\mathrm{c}}$, there exists a disc of radius $x_{\mathrm{in}}>0$ so that

$$
\rho_{\Omega}^{\mathrm{TF}}(x)=0, \quad \text { for any } 0 \leq x \leq x_{\mathrm{in}} .
$$

We also point out that by the rescalings (1.9) and (1.15), $\omega<\omega_{\mathrm{c}}$ holds if and only if $\Omega<\Omega_{\mathrm{c}}$, so that the conditions $|\log \varepsilon| \ll \omega<\omega_{\mathrm{c}}$ and $\Omega_{c} \leq \Omega \ll \varepsilon^{-4}$ cover all the possible asymptotic regimes between $\Omega_{\mathrm{c}_{1}}$ and $\Omega_{\mathrm{c}_{3}}$.

We can now formulate the result about the energy asymptotics of $E^{\mathrm{GP}}$ :

Theorem 1.1 (GP ground state energy).

If $|\log \varepsilon| \ll \omega<\omega_{\mathrm{c}}$ as $\varepsilon \rightarrow 0$, then

$$
E_{\omega}^{\mathrm{GP}}=E_{\omega}^{\mathrm{TF}}+\frac{1}{2} \omega\left|\log \left(\varepsilon^{2} \omega\right)\right|(1+o(1)),
$$

while, if $\Omega_{\mathrm{c}} \leq \Omega \ll \varepsilon^{-4}$,

$$
E_{\Omega}^{\mathrm{GP}}=E_{\Omega}^{\mathrm{TF}}+\frac{1}{6} \Omega\left|\log \left(\varepsilon^{4} \Omega\right)\right|(1+o(1)) .
$$

As we have anticipated a transition takes place when the angular velocity gets larger than some critical value of order $\mathcal{O}\left(\varepsilon^{-1}\right)$ : A direct consequence of the asymptotics (1.37) and (1.38) (see, e.g., CPRY1, Proposition 3.1]) is that

$$
\left\|\left|\psi^{\mathrm{GP}}\right|^{2}-\rho^{\mathrm{TF}}\right\|_{2} \leq o(1)\left\|\rho^{\mathrm{TF}}\right\|_{\infty},
$$

i.e., the density $\left|\psi^{\mathrm{GP}}\right|^{2}$ is close in $L^{2}\left(\mathbb{R}^{2}\right)$ to the $\mathrm{TF}$ density $\rho^{\mathrm{TF}}$, but when $\Omega$ gets larger than $\Omega_{\mathrm{c}}$ the latter develops a hole and a macroscopic region where $\psi^{\mathrm{GP}}$ is exponentially small in $\varepsilon$ appears close to the center of the trap. Next proposition shows that the threshold of such a transition is precisely given by $\Omega_{\mathrm{c}}$, i.e.,

$$
\Omega_{\mathrm{C}_{2}}=(s k / \gamma)^{\frac{2}{s+2}} \Omega_{\mathrm{c}}^{\frac{s-2}{s+2}}, \quad \Omega_{\mathrm{c}}=2 \sqrt{\frac{2(s+2)}{\pi \gamma(s-2)}}\left(\frac{2}{s}\right)^{\frac{2}{s-2}} \varepsilon^{-1} .
$$

\footnotetext{
${ }^{4}$ Notice that by the scaling relation [1.21), $\Omega=\mathcal{O}\left(\varepsilon^{-1}\right)$ if and only if $\omega=\mathcal{O}\left(\varepsilon^{-1}\right)$.
} 
Proposition 1.1 (Emergence of a hole in $\psi^{\mathrm{GP}}$ ).

If, as $\varepsilon \rightarrow 0, \Omega \geq \Omega_{0} \varepsilon^{-1}$ with $\Omega_{0}>\varepsilon \Omega_{\mathrm{c}}$ there exists $0<x_{\text {hole }}<1$ such that

$$
\left|\psi^{\mathrm{GP}}(\mathbf{x})\right|=\mathcal{O}\left(\varepsilon^{\infty}\right)
$$

for any $\mathrm{x}$ such that $0 \leq x \leq x_{\text {hole }}$.

We conclude this section by stating a result about the vortex distribution and showing that throughout the parameter region $|\log \varepsilon| \leq \omega \lesssim \varepsilon^{-1}$ (resp. $\varepsilon^{-1} \lesssim \Omega \ll \varepsilon^{-4}$ ), the vorticity of $\psi_{\omega}^{\mathrm{GP}}$ (resp. $\psi_{\Omega}^{\mathrm{GP}}$ ) is uniformly distributed on a length scale $\Omega^{-1 / 2}$ inside $\operatorname{supp}\left(\rho_{\omega}^{\mathrm{TF}}\right)\left(\right.$ resp. $\operatorname{supp}\left(\rho_{\Omega}^{\mathrm{TF}}\right)$ ) (see also the Remark after Theorem 1.2). For technical reasons, however, we are not able to prove the result in the whole support of $\rho^{\mathrm{TF}}$, but we have to restrict the analysis to the slightly smaller region

$$
\mathcal{R}_{\text {bulk }}:=\left\{\mathrm{x} \in \mathbb{R}^{2}: x_{<} \leq x \leq x_{>}\right\},
$$

where for some positive parameter $\beta:=\beta(\varepsilon, \Omega)$ such that $\beta \rightarrow 0$ as $\varepsilon \rightarrow 0$ (see (2.76) for its precise definition)

$$
x_{<}:=\left\{\begin{array}{ll}
0, & \text { if }|\log \varepsilon| \ll \omega<\omega_{\mathrm{c}}, \\
x_{\mathrm{in}}+\beta(\varepsilon \Omega)^{2 / 3}, & \text { if } \Omega_{\mathrm{c}} \leq \Omega \ll \varepsilon^{-4},
\end{array} \quad x_{>}:=x_{\mathrm{out}}-\beta(\varepsilon \Omega)^{2 / 3} .\right.
$$

Here $x_{\text {in }}$ and $x_{\text {out }}$ are the inner and outer radii of the support of the TF density. The definitions (1.43) imply that

$$
\left|\operatorname{supp}\left(\rho^{\mathrm{TF}}\right) \backslash \mathcal{R}_{\text {bulk }}\right|=\mathcal{O}\left(\beta\left(1+(\varepsilon \Omega)^{2 / 3}\right)^{-1}\right) \ll\left|\operatorname{supp}\left(\rho^{\mathrm{TF}}\right)\right|
$$

and

$$
\left\|\psi^{\mathrm{GP}}\right\|_{L^{2}\left(\mathcal{R}_{\mathrm{bulk}}\right)}=1-o(1)
$$

i.e., $\mathcal{R}_{\text {bulk }}$ contains the bulk of the mass of $\psi^{\mathrm{GP}}$.

In the following theorem, we describe the vorticity distribution in the bulk of the condensate for rotation speeds between the first and third critical speeds. As it is usual in the Ginzburg-Landau (GL) theory, this is done by investigating the asymptotic properties of a certain vorticity measure whose definition is part of the statement of the theorem. Note that we do not tackle the question of the precise location of the vortices, which should be on the sites of a hexagonal lattice. Proving this fact rigorously remains a major open problem in both GP and GL theories, see SS3] for the most recent results in this direction.

In the statement of the theorem we use the notation $\mathcal{B}(\mathbf{x}, \rho)$ for a closed disc ('ball') with center at $\mathbf{x}$ and radius $\rho$.

Theorem 1.2 (Uniform distribution of vorticity). If $|\log \varepsilon| \ll \omega \lesssim \varepsilon^{-1}$ or $\varepsilon^{-1} \lesssim \Omega \ll \varepsilon^{-4}$ as $\varepsilon \rightarrow 0$, there exists a finite family of disjoint balls $\left\{\mathcal{B}_{i}\right\}:=$ $\left\{\mathcal{B}\left(\mathbf{x}_{i}, \varrho_{i}\right)\right\} \subset \mathcal{R}_{\text {bulk }}, i=1, \ldots, N$, such that

1. $\varrho_{i} \leq \mathcal{O}\left(\omega^{-1 / 2}+\Omega^{-1 / 2}\right), \sum \varrho_{i}^{2} \leq\left(1+(\varepsilon \Omega)^{2 / 3}\right)^{-1}$;

2. $\left|\psi^{\mathrm{GP}}\right|>0$ on $\partial \mathcal{B}_{i}, i=1, \ldots, N$.

Moreover, setting $d_{i}:=\operatorname{deg}\left\{\psi^{\mathrm{GP}}, \partial \mathcal{B}_{i}\right\}$ and defining the vorticity measure as

$$
\nu:=2 \pi \sum_{i=1}^{N} d_{i} \delta\left(\mathbf{x}-\mathbf{x}_{i}\right)
$$

then, for any set $\mathcal{S} \subset \mathcal{R}_{\text {bulk }}$ such that $|\mathcal{S}| \gg \Omega^{-1}\left|\log \left(\varepsilon^{4} \Omega\right)\right|^{2}$ as $\varepsilon \rightarrow 0$,

$$
\frac{\nu(\mathcal{S})}{\Omega|\mathcal{S}|} \underset{\varepsilon \rightarrow 0}{\longrightarrow} 1 \text {. }
$$


Remark 1.1 (Vortex balls)

By a closer inspection of the proof of the above Theorem 1.2, one can realize that there is more information contained in the family of balls $\left\{\mathcal{B}_{i}\right\}$ : Each ball might contain a very large number of vortices but almost all vortices are enclosed by those balls. More precisely, there exists a 'good region' in the condensate where all the possible zeros of $\psi^{\mathrm{GP}}$ are contained in $\cup \mathcal{B}_{i}$ and outside this set $\psi^{\mathrm{GP}}$ is vortex free. There is also a complementary 'bad region' where we are not able to prove such a thing, but a key point of the proof of Theorem 1.2 is that the good region covers most of the condensate, i.e., the ratio between the areas of the bad and good regions is $o(1)$ as $\varepsilon \rightarrow 0$.

\subsection{The Regime $\Omega \propto \varepsilon^{-4}$ : Transition to a Giant Vortex State}

In this section we consider $\Omega$ given as

$$
\Omega=\frac{\Omega_{0}}{\varepsilon^{4}}
$$

where $\Omega_{0}$ is a fixed constant while $\varepsilon \rightarrow 0$.

A 'giant vortex state', that turns out to be an excellent approximation to the ground state for large $\Omega_{0}$, is obtained by minimizing the GP functional over functions of the form $f(\mathbf{x}) e^{i\lfloor\Omega\rfloor \vartheta}$ with $f$ real. The giant vortex energy functional is given by

$$
\mathcal{E}^{\mathrm{gv}}[f]:=\mathcal{E}_{\Omega}^{\mathrm{GP}}\left[f e^{i\lfloor\Omega\rfloor \vartheta}\right]=\int_{\mathbb{R}^{2}} \mathrm{~d} \mathbf{x}\left\{\frac{1}{2}|\nabla f|^{2}+\Omega^{2} U(x) f^{2}+\varepsilon^{-2} f^{4}\right\}
$$

where

$$
U(x):=\frac{1}{2}\lfloor\Omega\rfloor^{2} \Omega^{-2} x^{-2}+\frac{1}{2} x^{2}+\frac{1}{s} \gamma\left(x^{s}-1\right)-\frac{1}{2} \gamma\left(x^{2}-1\right)-\lfloor\Omega\rfloor \Omega^{-1} .
$$

By standard arguments (see Proposition 3.3) one can show that there exists a unique minimizer $g_{\mathrm{gv}}$ of (1.49) in the domain

$$
\mathscr{D}^{\mathrm{gv}}:=\left\{f \in H^{1}\left(\mathbb{R}^{2}\right): f=f^{*} \geq 0, x^{s} f^{2} \in L^{1}\left(\mathbb{R}^{2}\right),\|f\|_{2}=1\right\} .
$$

In addition it is easily seen that $g_{\mathrm{gv}}$ is radial and does not vanish except at the origin. We also set

$$
E^{\mathrm{gv}}:=\inf _{f \in \mathscr{D}_{\mathrm{gv}}} \mathcal{E}^{\mathrm{gv}}[f]
$$

The leading order of the GP energy and density will be given with very good accuracy by the minimization of the simpler giant vortex energy functional. It is thus of importance to understand the properties of the latter. When $\Omega \ll \varepsilon^{-4}$, its minimization leads to a profile that is to leading order of TF-type as discussed in the preceding subsection. A salient feature of the regime (1.48) is the fact that, when $\Omega_{0}$ gets large, the giant vortex density $g_{\mathrm{gv}}$ changes from a TF profile to a gaussian distribution centered on the circle of radius 1 . This is most conveniently understood by using an auxiliary one-dimensional energy functional (recall that $g_{\mathrm{gv}}$ is radial)

$$
\begin{aligned}
& \mathcal{E}^{\operatorname{aux}}[f]=2 \pi \int_{\mathbb{R}} \mathrm{d} x\left\{\frac{1}{2}\left|f^{\prime}\right|^{2}+\frac{1}{2} \Omega^{2} \alpha^{2}(x-1)^{2} f^{2}+\varepsilon^{-2} f^{4}\right\}= \\
& 2 \pi \Omega \int_{\mathbb{R}} \mathrm{d} y\left\{\frac{1}{2}\left|\hat{f}^{\prime}\right|^{2}+\frac{1}{2} \alpha^{2} y^{2} \hat{f}^{2}+\Omega_{0}^{-1 / 2} \hat{f}^{4}\right\},
\end{aligned}
$$

where

$$
\alpha:=\sqrt{4+2(s-2)}
$$

\footnotetext{
${ }^{5}$ Here $\left.L\right\rfloor$ stands for the integer part.
} 
One obtains $\mathcal{E}^{\text {aux }}$ from (1.49) by expanding the potential around its minimum at $x=1$ and neglecting terms beyond quadratic in the expansion. The variable transformation $y=\Omega^{1 / 2}(x-1), \hat{f}(y)=\Omega^{-1 / 4} f(x)$ is then used to blow-up to the characteristic size of the domain where the density is confined. It is clear that all three terms in (1.52) are of the same order of magnitude but the importance of the last term diminishes with increasing $\Omega_{0}$. The quadratic part of the energy is simply the energy functional of the harmonic oscillator, so without the last term the minimizer is the Gaussian

$$
g_{\text {osc }}(y)=\pi^{-1 / 4} \alpha^{1 / 4} \exp \left\{-\frac{1}{2} \alpha y^{2}\right\} .
$$

We expect and prove (see Section [3.2) that $g_{\mathrm{gv}}$ is close (after rescaling) to this function when $\Omega_{0}$ is large. This behavior of the density profile is very different from that given by the TF theory discussed before. The fact that the transition to a gaussian profile occurs in the same parameter regime as the transition to a giant vortex phase is at the origin of several new features of the physics of the third phase transition in a soft potential as compared to the theory in a flat trap. This does not seem to have been noticed in the literature before: As far as we know, the profile is always assumed to be of TF type in previous papers dealing with the transition to a giant vortex state, with the exception of [R1].

To begin with, it is more delicate to define the bulk of the condensate in the regime $\Omega_{0}$ large. The gaussian mass distribution has much slower decay and longer tails than a TF-like profile. As a consequence the mass is much less concentrated and it is necessary to consider an annulus of thickness much larger than the characteristic scale $\Omega^{-1 / 2}=\Omega_{0}^{-1 / 2} \varepsilon^{2}$ of the gaussian distribution:

$$
\mathcal{A}_{\text {bulk }}:=\left\{\mathbf{x} \in \mathbb{R}^{2}: 1-c|\log \varepsilon|^{1 / 2} \Omega^{-1 / 2} \leq x \leq 1+c|\log \varepsilon|^{1 / 2} \Omega^{-1 / 2}\right\}, \quad c<\sqrt{\frac{2}{\alpha}} .
$$

Our main result about the regime (1.48) is the following:

Theorem 1.3 (Absence of vortices in the bulk).

If $\Omega$ is given by (1.48), there exists a finite constant $\bar{\Omega}_{0}$ such that for any $\Omega_{0}>\bar{\Omega}_{0}$, no minimizer $\psi^{\mathrm{GP}}$ has a zero inside $\mathcal{A}_{\mathrm{bulk}}$ if $\varepsilon$ is sufficiently small.

More precisely, for any $\mathrm{x} \in \mathcal{A}_{\mathrm{bulk}}$,

$$
\left|\psi^{\mathrm{GP}}(\mathbf{x})\right|=g_{\mathrm{gv}}(x)\left(1+\mathcal{O}\left(|\log \varepsilon|^{-a}\right)\right)
$$

for any $a>0$.

The estimate (1.56) justifies the notation $\mathcal{A}_{\text {bulk }}$ : Indeed we have

$$
\int_{\mathcal{A}_{\mathrm{bulk}}} \mathrm{d} \mathbf{x}\left|\psi^{\mathrm{GP}}\right|^{2}=1-o(1)
$$

because such an estimate holds true for $g_{\mathrm{gv}}$, so $\mathcal{A}_{\text {bulk }}$ contains the bulk of the mass. Theorem 1.3 then characterizes the transition to a giant vortex phase where the bulk of the condensate resides in an annulus free of vortices but with a macroscopic circulation around the origin. Recalling the results of Theorem 1.2 we deduce that the third phase transition occurs when $\Omega$ is of order $\varepsilon^{-4}$. Indeed, for speeds much smaller than this threshold, vortices do occur in the annular bulk, whereas they are absent if $\Omega$ is a sufficiently large multiple of $\varepsilon^{-4}$.

The core of the proof of Theorem 1.3 is a detailed analysis of the asymptotic behavior of the GP ground state energy, leading to the following estimate:

Theorem 1.4 (Ground state energy asymptotics). If $\Omega$ is given by (1.48) with $\Omega_{0}>\bar{\Omega}_{0}$ as in Theorem 1.3 , then as $\varepsilon \rightarrow 0$

$$
E^{\mathrm{GP}}=E^{\mathrm{gv}}+\mathcal{O}\left(|\log \varepsilon|^{9 / 2}\right) .
$$


We are also able to estimate the degree of the GP minimizer, ensuring that there is a macroscopic phase circulation around the central low-density hole. This is the content of the following theorem.

Theorem 1.5 (Asymptotics for the degree). If $\Omega$ is given by (1.48) with $\Omega_{0}>\bar{\Omega}_{0}$ as in Theorem 1.3 and $R$ is any radius satisfying

$$
R=1+\mathcal{O}\left(\Omega^{-1 / 2}\right)
$$

then as $\varepsilon \rightarrow 0$

$$
\operatorname{deg}\left(\psi^{\mathrm{GP}}, \partial B_{R}\right)=\Omega+\mathcal{O}\left(\Omega_{0}|\log \varepsilon|^{9 / 4}\right) .
$$

As already mentioned, the physics of the giant vortex phase transition in a homogeneous trap turns out to differ markedly from the corresponding regime in a flat trap that was studied in CRY, CPRY1, R2. As a result, the technique of proof is different and does not rely on any construction of vortex balls. The remaining part of this subsection aims at elucidating the main steps in the proof. To bring out the salient points technical details are skipped and most of the following equations will be written in a simplified form without the remainder terms. We remark also that the method to be presented is simpler than that in R1 and leads to sharper results, although some ideas are common to both papers.

As a starter let us note that, in the soft potentials $(s<\infty)$ we consider, the centrifugal force does not squeeze the condensate against a hard wall as in the flat potential $(s=\infty)$ considered in CPRY1 although it still concentrates the scaled density in an annulus around $x=1$. The soft potential, however, allows more spreading of the matter density than a trap with a hard wall, so the density is lower and vortices therefore energetically less costly. This explains the fact that the third critical speed is considerably larger in a homogeneous potential than could be expected from the analysis of the flat trap model.

It had been recognized before $\mathrm{R} 1$ that for extreme rotation speeds the density profile would be gaussian, but when studying the giant vortex transition a key difficulty originates from the fact that the change from the TF profile to the gaussian distribution takes place in the same parameter regime as the one where vortices are expelled from the bulk of the condensate. In a sense two distinct transitions happen at the same time in the regime (1.48) and this is the main reason why the analysis we perform in this paper is rather different from CRY, CPRY1.

Indeed, in CRY, CPRY1] the potential vortex cores were much smaller than the width of the annular bulk of the condensate and vortices disappeared for energetic reasons. To analyze this phenomenon precisely we had to adapt the method of vortex balls originally developed in the GL theory (see [SS2] and references therein) and used to evaluate $\Omega_{\mathrm{c}_{1}}$ IM1, AJR.

In a homogeneous trap the cores of potential vortices turn out to be comparable in size to the width of the annular bulk when $\Omega \sim \Omega_{\mathrm{c}_{3}}$. It is thus not clear whether they disappear because of their energy cost or are simply expelled from the condensate because they are too large to fit in. This explains why we have not been able to give a precise estimate of the third critical speed: Potential vortices are too large to treat them using the GL technology. Their energy cost can not be evaluated as accurately as in CRY, R2 and we have to rely on coarser estimates.

The starting point and the basic methodology is however reminiscent to that employed in CRY, CPRY1 and in works dealing with the first critical speed in GP theory AAB, IM1, IM2. We first decouple the energy functional, an idea originating in [LM] and used repeatedly in the literature. Actually it is more convenient to restrict the integration domain before performing the energy decoupling. For technical reasons it is not sufficient to work on $\mathcal{A}_{\text {bulk }}$ (defined by Eq. (1.55)) and we consider instead a larger domain

$$
\mathcal{A}_{\eta_{\varepsilon}}=\left\{\mathrm{x} \in \mathbb{R}^{2}: 1-\eta_{\varepsilon} \varepsilon^{2} \leq x \leq 1+\eta_{\varepsilon} \varepsilon^{2}\right\}
$$

where we have some freedom in the choice of $\eta_{\varepsilon} \gg 1$. It should in any case satisfy the condition $\eta_{\varepsilon} \gg|\log \varepsilon|^{1 / 2}$ in order for $\mathcal{A}_{\eta_{\varepsilon}}$ to contain $\mathcal{A}_{\text {bulk }}$ but since we shall also need to consider $\mathcal{A}_{\sqrt{\eta}}$ we even require $\eta_{\varepsilon} \gg|\log \varepsilon|$. If $\eta_{\varepsilon}$ is chosen large enough, both the true GP minimizer and the giant vortex density profile are exponentially small in $\varepsilon$ outside $\mathcal{A}_{\eta_{\varepsilon}}$ and one can restrict the analysis to this annulus 
at the price of negligible remainders. On the other hand, the proof of energy estimates and the absence of vortices requires that $\eta_{\varepsilon}$ is not too large and we shall eventually choose $\eta_{\varepsilon}=|\log \varepsilon|^{3 / 2}$, cf. Eq. (3.104).

By defining a giant vortex functional reduced to $\mathcal{A}_{\eta_{\varepsilon}}$ and minimizing it, we obtain an energy $E_{\eta_{\varepsilon}}^{\mathrm{gv}}$ and a profile $g_{\eta_{\varepsilon}}$ supported in the annulus $\mathcal{A}_{\eta_{\varepsilon}}$. We then write any test function $\psi$ as

$$
\psi(\mathbf{x})=g_{\eta_{\varepsilon}}(x) e^{i\lfloor\Omega\rfloor \vartheta} v(\mathbf{x}),
$$

which defines $v$ on $\mathcal{A}_{\eta_{\varepsilon}}$. Since $g_{\eta_{\varepsilon}}$ is without zeros, the function $v$ contains all possible zeros of $\psi$ in the annulus. The variational equation for $g_{\eta_{\varepsilon}}$ leads to

$$
\mathcal{E}^{\mathrm{GP}}[\psi] \simeq E_{\eta_{\varepsilon}}^{\mathrm{gv}}+\mathcal{E}_{\eta_{\varepsilon}}[v]
$$

with

$$
\mathcal{E}_{\eta_{\varepsilon}}[v]=\int_{\mathcal{A}_{\eta_{\varepsilon}}} \mathrm{d} \mathbf{x} g_{\eta_{\varepsilon}}^{2}\left\{\frac{1}{2}|\nabla v|^{2}-\tilde{\mathbf{B}} \cdot(i v, \nabla v)+\varepsilon^{-2} g_{\eta_{\varepsilon}}^{2}\left(1-|v|^{2}\right)^{2}\right\}
$$

where $\tilde{\mathbf{B}}=\left(\Omega x-\lfloor\Omega\rfloor x^{-1}\right) \mathbf{e}_{\vartheta}$ and $(i v, \nabla v)=\frac{\mathrm{i}}{2}\left(v \nabla v^{*}-v^{*} \nabla v\right)$. It is plausible that if we write

$$
\psi^{\mathrm{GP}}(\mathbf{x})=g_{\eta_{\varepsilon}}(x) e^{i\lfloor\Omega\rfloor \vartheta} u(\mathbf{x}),
$$

then the function $u$ defined on $\mathcal{A}_{\eta_{\varepsilon}}$ should minimize $\mathcal{E}_{\eta_{\varepsilon}}$ under the constraint

$$
\int_{\mathcal{A}_{\eta_{\varepsilon}}} \mathrm{d} \mathbf{x} g_{\eta_{\varepsilon}}^{2}|u|^{2}=1
$$

This is not fully rigorous because some remainder terms in (1.62) are neglected, most of which can be controlled using the exponential smallness of $\psi^{\mathrm{GP}}$ outside of $\mathcal{A}_{\eta_{\varepsilon}}$. Nevertheless, studying the reduced problem of minimizing $\mathcal{E}_{\eta_{\varepsilon}}$ under the constraint (1.65) gives some valuable insight on the full proof we are to develop in Section 3 ,

The appropriate trial function is given by $v=1$, which corresponds to a pure giant vortex ansatz. This gives

$$
\mathcal{E}_{\eta_{\varepsilon}}[u] \leq 0
$$

and the gist of the proof is the obtainment of the corresponding lower bound, proving that the giant vortex trial function optimizes the energy up to small remainders. To this end one needs to control the only possibly negative contribution originating in the second term of (1.63). This is most conveniently done using a potential function defined as in [AAB, CRY]:

$$
F(x)=\int_{1-\varepsilon^{2} \eta_{\varepsilon}}^{x} \mathrm{~d} t\left(\Omega t-\lfloor\Omega\rfloor t^{-1}\right) g_{\eta_{\varepsilon}}^{2}(t) .
$$

This function satisfies $\nabla^{\perp} F=g_{\eta_{\varepsilon}}^{2} \tilde{\mathbf{B}}$ and vanishes on the inner boundary of $\mathcal{A}_{\eta_{\varepsilon}}$. Using Stokes' formula on the second term of (1.63) leads to

$$
\mathcal{E}_{\eta_{\varepsilon}}[u]=\int_{\mathcal{A}_{\eta_{\varepsilon}}} \mathrm{d} \mathbf{x}\left\{\frac{1}{2} g_{\eta_{\varepsilon}}^{2}|\nabla u|^{2}+F \mu+\varepsilon^{-2} g_{\eta_{\varepsilon}}^{4}\left(1-|u|^{2}\right)^{2}\right\}-F\left(1+\varepsilon^{2} \eta_{\varepsilon}\right) \int_{\partial B_{1+\varepsilon^{2} \eta_{\varepsilon}}} \mathrm{d} \sigma\left(i u, \partial_{\tau} u\right) .
$$

The quantity $\mu$ is defined as

$$
\mu:=\operatorname{curl}(i u, \nabla u)
$$

and is often referred to as the vorticity measure because in many situations involving vortices one can prove that such a measure is close to measure of the form (1.46): a sum of Dirac masses counting the vortices of $u$ with multiplicity. It is not completely clear that $\mu$ deserves such a denomination in the 
regime we consider: As already emphasized, the cores of potential vortices 6 are too large to employ the jacobian estimate of [JS] that would put the comparison with a sum of Dirac masses on a rigorous ground. For illustration we shall employ the terminology nevertheless. It is justified by the fact that only the term involving $\mu$ in $\mathcal{E}_{\eta_{\varepsilon}}[u]$ could favor the nucleation of vortices. Indeed the other bulk terms are positive and the boundary term should morally be negligible as we now explain.

Since $\Omega$ is very large it is plausible that one can replace it with its integer part at negligible cost. Also the density profile should be almost symmetric about the radius 1 . If it were exactly symmetric, $F\left(1+\eta_{\varepsilon} \varepsilon^{2}\right)$ would be extremely smal 7 and the boundary term in (1.67) could be neglected, reducing the proof of the lower bound to the estimate of the second term in (1.67).

To deal with the term $\int_{\mathcal{A}_{\eta_{\varepsilon}}} F \mu$ we first remark that due to the Cauchy-Schwarz inequality we have

$$
|\mu| \leq|\nabla u|^{2}
$$

pointwise, and in the regime (1.48) $g_{\eta_{\varepsilon}}$ and $F$ are of the same order of magnitude. It is thus plausible that $g_{\eta_{\varepsilon}}^{2}|\nabla u|^{2}$ can control $F \mu$ pointwise. This is in marked contrast to the situation in a flat trap where $g_{\eta_{\varepsilon}}$ is much smaller than $F$, making the method of vortex balls indispensable to improve the precision of the estimates.

For $\Omega_{0}$ large enough, $g_{\eta_{\varepsilon}}$ is approximately gaussian, so that one can use the ansatz (1.54) to compute $F$ and obtain

$$
|F(x)| \leq \alpha^{-1} g_{\eta_{\varepsilon}}^{2}(x)
$$

for any $\mathrm{x} \in \mathcal{A}_{\text {bulk }}$ and $\Omega_{0}$ large enough. As $\alpha>2$ (see (1.53)), combining (1.69) with (1.70) we can conclude that the kinetic energy term in (1.67) dominates the term involving the vorticity measure, which leads to a lower bound confirming that the giant vortex ansatz is optimal (up to negligible remainders). As a by-product of the proof one can also obtain an estimate of the third term in (1.63) that contradicts the presence of vortices in $\mathcal{A}_{\text {bulk }}$ : The technique, involving an estimate of $\|\nabla u\|_{L^{\infty}}$, originates in [BBH] and has already been used in the flat trap case CRY, CPRY1.

What remains to be understood is the way in which one can justify the approximations made above. We have assumed that $\Omega$ could be replaced by its integer part. This is harmless and the corresponding remainder terms are ignored in what follows. More importantly we have assumed that

- $g_{\eta_{\varepsilon}}$ is a radial Gaussian centered on $x=1$ for $\Omega_{0}$ large enough, which allows to prove (1.70);

- in particular $g_{\eta_{\varepsilon}}$ is symmetric with respect to 1 so that the integration by parts in (1.67) produces only very small remainder terms.

It is fairly easy to prove that $g_{\eta_{\varepsilon}}$ is close to a Gaussian around the center of the bulk profile where the matter density is large enough. However, we need to justify the pointwise estimate (1.70) in a domain that is much larger than the bulk, including a region where the density $g_{\eta_{\varepsilon}}$ is extremely small. To obtain an appropriate lower bound to $g_{\eta_{\varepsilon}}$, a subsolution is constructed for the corresponding variational equation. For the method to apply it is necessary to restrict to a smaller annulus: We are able to prove that $g_{\eta_{\varepsilon}}$ is pointwise close to the optimal Gaussian only in $\mathcal{A}_{\sqrt{\eta_{\varepsilon}}}$. The region $\mathcal{A}_{\eta_{\varepsilon}} \backslash \mathcal{A}_{\sqrt{\eta_{\varepsilon}}}$ is dealt with 8 using the exponential smallness of $\psi^{\mathrm{GP}}$ in the low density hole.

As for the boundary term in (1.67) one can prove that $g_{\eta_{\varepsilon}}$ is to a very good approximation symmetric around $x=1$. Indeed, as we have explained, the profile becomes gradually gaussian when $\Omega_{0}$ is increased, because the weight of the quartic component of the energy decreases. The reason we have separated the

\footnotetext{
${ }^{6}$ The size of vortex cores can be estimated by taking it as a variational parameter in a trial function with prescribed zeros and minimizing the sum of the first and third terms in 1.63 .

${ }^{7} \mathrm{As} t-t^{-1}$ is to a very good approximation antisymmetric with respect to $1, F\left(1+\eta_{\varepsilon} \varepsilon^{2}\right)$ would reduce to the integral of an odd function on a domain symmetric with respect to the origin, which vanishes.

${ }^{8}$ There is some freedom in the choice of $\mathcal{A}_{\sqrt{\eta_{\varepsilon}}}$, the essential point being that we can prove the appropriate lower bound on $g_{\eta_{\varepsilon}}$ only in a smaller domain than $\mathcal{A}_{\eta_{\varepsilon}}$.
} 
discussion of the symmetry from that of the approximation by a Gaussian is that $g_{\eta_{\varepsilon}}$ is very close to a symmetric function even for $\Omega_{0}$ small where the gaussian approximation is not expected to be valid. In some sense $g_{\eta_{\varepsilon}}$ is much more symmetric than it is gaussian. To understand this, one can consider the energy functional (1.52) obtained by keeping only the quadratic term in the Taylor expansion of the potentials: After rescaling, the minimizer is symmetric for any $\Omega_{0}$ and one can prove that it approximates $g_{\eta_{\varepsilon}}$ much better 9 than the Gaussian (1.54).

Making use of this property we show that $F\left(1+\varepsilon^{2} \eta_{\varepsilon}\right)$ is indeed very small. Then one could hope to conclude that the boundary term is negligible if a proper estimate of the phase circulation

$$
\int_{\partial B_{1+\varepsilon^{2} \eta_{\varepsilon}}} \mathrm{d} \sigma\left(i u, \partial_{\tau} u\right)
$$

were available. This is however not easy to obtain because the integral is located in a region where $g_{\eta_{\varepsilon}}$ is very small, casting doubt on the possibility to prove any estimate of the term above using the kinetic energy $\frac{1}{2} \int_{\mathcal{A}_{\eta_{\varepsilon}}} g_{\eta_{\varepsilon}}^{2}|\nabla u|^{2}$.

To get around this point we modify our strategy as follows: Instead of the potential function (1.66) we use two different potentials $F_{1}, F_{2}$ vanishing respectively on $\partial B_{1-\varepsilon^{2} \eta_{\varepsilon}}$ and $\partial B_{1+\varepsilon^{2} \eta_{\varepsilon}}$. The first is used to calculate the part of the momentum term residing between the inner boundary of $\mathcal{A}_{\eta_{\varepsilon}}$ and the maximum point of $g_{\eta_{\varepsilon}}$, while we use the second in the complementary region, leading to a formula of the form

$$
\mathcal{E}_{\eta_{\varepsilon}}[u] \simeq \int_{\mathbb{R}^{2}} \mathrm{~d} \mathbf{x}\left\{\frac{1}{2} g_{\eta_{\varepsilon}}^{2}|\nabla u|^{2}+\left(F_{1}+F_{2}\right) \mu+\varepsilon^{-2} g_{\eta_{\varepsilon}}^{4}\left(1-|u|^{2}\right)^{2}\right\}-\left(F_{1}(1)-F_{2}(1)\right) \int_{\partial \mathcal{B}_{1}} \mathrm{~d} \sigma\left(i u, \partial_{\tau} u\right),
$$

with the convention that $F_{1}=0$ for $r \geq 1$ and $F_{2}=0$ for $r \leq 1$. In some sense we are using a discontinuous potential, which has the effect to produce a boundary term on $\partial B_{1}$, i.e., in the middle of the bulk 10 . An important point is that $\left|F_{1}+F_{2}\right|<\frac{1}{2} g_{\eta_{\varepsilon}}^{2}$ when $\Omega_{0}$ is large, which means that the analysis of the bulk terms can be carried out exactly as sketched above.

On the other hand the jump of the potential function $F_{1}(1)-F_{2}(1)$ is roughly speaking given by $F\left(1+\varepsilon^{2} \eta_{\varepsilon}\right)$ with $F$ defined in (1.66) and is thus very small because of the symmetry of $g_{\eta_{\varepsilon}}$. The key point is that this information can be combined with an efficient estimate of the phase circulation of $u$ on $\partial B_{1}$. Indeed, the boundary term is now located where the matter density is large, which allows to control the phase circulation of $u$ in terms of the kinetic term in the energy (see Lemma 3.5). This control also leads to the degree estimate of Theorem 1.5 once $u$ has been proved to be vortex free.

The trick of using a discontinuous potential function to make boundary terms tractable in the study of functionals such as (1.62) seems to be new. In situations considered before [AAB, CRY, CPRY1, the TF-like profile allowed to employ other methods for estimating boundary terms. These fail in the context of the present paper due to the long tails of the approximately gaussian density profile.

\subsection{Rotational Symmetry Breaking}

The nucleation of vortices in a rotating superfluid trapped in a radial potential has an important connection with the symmetry properties of the ground state. For example, when $\Omega_{\text {rot }}$ is kept below the first critical speed, the ground state is expected to be unique and rotationally symmetric. This has recently been proved in AJR at least in the TF limit: The ground state for $\Omega_{\mathrm{rot}}<\Omega_{\mathrm{c}_{1}}$ is equal to the ground state without rotation, i.e., for $\Omega_{\text {rot }}=0$, which is a radial function. When there is only one vortex in the superfluid, it will sit at the center of the trap and the ground state, although not radial any longer, is still an eigenfunction of the angular momentum, i.e., it is of the form $f(r) e^{i d \vartheta}$ with $f$ a real-valued function and $d \in \mathbb{Z}$.

\footnotetext{
${ }^{9}$ One can compare the remainders in 3.43 and 3.67 below.

${ }^{10}$ More precisely the jump is at the maximum point of the profile, which is close to 1 but not exactly equal to it.
} 
On the other hand the occurrence of more than one vortex in the GP minimizer $\psi^{\mathrm{GP}}$ is automatically associated with a breaking of the rotational symmetry of the functional at the level of the ground state (see, e.g., Seir, CDY1 for a detailed discussion of this phenomenon). Therefore Theorem 1.2 guarantees that rotational symmetry is actually broken for any $|\log \varepsilon| \ll \omega, \Omega \ll \varepsilon^{-4}$, since the bulk of the condensate is filled with a large number of vortices, namely of the order of the angular velocity $\omega$ or $\Omega$.

However, the giant vortex transition described in Section 1.3 seems to suggest a restoration of the symmetry under rotations: For instance the proof of Theorem 1.4 shows that a giant vortex trial function with all the vorticity concentrated at the origin can reproduce the GP ground state energy up to subleading order corrections. In addition the bulk of the condensate is vortex free (see Theorem 1.3) so all vorticity must reside in a region where the density is low.

In the following theorem we prove that the rotational symmetry is never restored even though the angular velocity crosses the giant vortex threshold. This suggests that there is a nontrivial distribution of vortices in the low density hole and it is an interesting open problem to locate them, or else find other reasons for the symmetry breaking.

Theorem 1.6 (Rotational symmetry breaking).

If $\Omega \gtrsim \varepsilon^{-4}$, no minimizer $\psi^{\mathrm{GP}}$ of the GP functional is an eigenfunction of angular momentum for $\varepsilon$ sufficiently small.

Note that the theorem is stated for $\Omega \gtrsim \varepsilon^{-4}$, i.e., in the giant vortex regime. This is the regime where one could a priori hope that the ground state of the theory is exactly of the form $f(r) e^{i d \vartheta}$.

The same result has already been proven for smaller angular velocities in [Seir, Theorem 2]: Assume for instance that $|\log \varepsilon| \lesssim \omega \ll \varepsilon^{-1}$, then it is not difficult to realize that the total winding number of any minimizer must be $\mathcal{O}(\omega)$. Therefore if one picks some finite $d>s / 2+1$, Theorem 2 in [Seir] proves the instability of any symmetric vortex of degree $n=\mathcal{O}(\omega)$ : Condition (2.29) in Seir is indeed always satisfied for $\varepsilon$ sufficiently small, since the chemical potential is of order $\mathcal{O}\left(\varepsilon^{-2}\right)$, whereas the r.h.s of (2.29) is $\mathcal{O}\left(\omega^{2}\right)$.

\subsection{Organization of the Paper}

The proofs of the results stated above are given in the following sections. The vortex lattice regime is discussed in Section 2, beginning in Sect. 2.1 with a comparison between the TF energy and density with those of an energy functional where the vector potential responsible for the appearance of vortices has been dropped. Section 2.2 contains the proof of upper and lower bounds to the vortex contributions to the energy. The uniform distribution of vorticity is proved in Section 2.3.

Section 3 is concerned with the giant vortex transition. After some preliminary estimates on the ground state energy and minimizing wave functions in Sect. 3.1, we study in Sect. 3.2 the giant vortex density profile. The essential results here are the estimates on the deviation from a Gaussian (Proposition 3.5) and from another profile symmetric about $x=1$ (Proposition 3.7). The basic energy estimates are given in Sect. 3.3 and in Sect. 3.4 they are combined with gradient estimates to establish the giant vortex transition.

Finally, breaking of rotational symmetry is proved in Section 4.

\section{The Regime $|\log \varepsilon| \ll \omega, \Omega \ll \varepsilon^{-4}$}

\subsection{The TF Energies and Densities}

We start by summing up some important information about the minimization of the TF functionals $\mathcal{E}_{\omega}^{\mathrm{TF}}$ and $\mathcal{E}_{\Omega}^{\mathrm{TF}}$ (Equations (1.28) and (1.33) ): We often refer to CDY2 for a detailed analysis and omit here the proof details. 
The unique TF minimizer of the functional (1.28) for $|\log \varepsilon| \ll \omega \lesssim \varepsilon^{-1}$ is the density (1.30), i.e.,

$$
\rho_{\omega}^{\mathrm{TF}}(x)=\frac{1}{2}\left[\varepsilon^{2} \mu_{\omega}^{\mathrm{TF}}-x^{s}+\frac{1}{2} \gamma \varepsilon^{2} \omega^{2} x^{2}\right]_{+}
$$

with $\mu_{\omega}^{\mathrm{TF}}=E^{\mathrm{TF}}+\varepsilon^{-2}\left\|\rho_{\omega}^{\mathrm{TF}}\right\|_{2}^{2}$. The support of $\rho_{\omega}^{\mathrm{TF}}$ is always a compact set since $s>2$, and there exists a critical value of the angular velocity $\omega_{\mathrm{c}}=\mathcal{O}\left(\varepsilon^{-1}\right)$ such that

$$
\operatorname{supp}\left(\rho_{\omega}^{\mathrm{TF}}\right)= \begin{cases}\left\{\mathbf{x} \in \mathbb{R}^{2}: x \in\left[0, x_{\mathrm{out}}\right]\right\}, & \text { if } \omega \leq \omega_{\mathrm{c}} \\ \left\{\mathbf{x} \in \mathbb{R}^{2}: x \in\left[x_{\mathrm{in}}, x_{\text {out }}\right]\right\}, & \text { if } \omega>\omega_{\mathrm{c}}\end{cases}
$$

with $x_{\text {in }}>0$. It is given by

$$
\omega_{\mathrm{c}}=2^{\frac{s}{s+2}} \gamma^{-1 / 2}\left(\frac{2(s+2)}{\pi(s-2)}\right)^{\frac{s+2}{2(s-2)}} \varepsilon^{-1}
$$

If $\omega=\omega_{\mathrm{c}}$, the outer radius is

$$
x_{\text {out }}=\left(\frac{1}{2} \gamma \varepsilon^{2} \omega_{\mathrm{c}}^{2}\right)^{\frac{1}{s-2}} .
$$

For generic $\omega$ neither $x_{\text {in }}$ nor $x_{\text {out }}$ are explicitly given but can be obtained by solving the equations $\rho_{\omega}^{\mathrm{TF}}\left(x_{\mathrm{in} / \text { out }}\right)=0$. Both radii are increasing functions of $\omega$, whereas both $\mu_{\omega}^{\mathrm{TF}}$ and $x_{\mathrm{out}}-x_{\mathrm{in}}$, i.e., the width of $\operatorname{supp}\left(\rho_{\omega}^{\mathrm{TF}}\right)$, decrease as $\omega$ increases.

When $\omega$ becomes larger so that $\omega \gtrsim \varepsilon^{-1}$ (which is the same as $\Omega \gtrsim \varepsilon^{-1}$ ) we use the definition (1.33) for the TF functional. The TF minimizer is in this case

$$
\rho_{\Omega}^{\mathrm{TF}}(x):=\frac{1}{2}\left[\varepsilon^{2} \mu_{\Omega}^{\mathrm{TF}}-\gamma \varepsilon^{2} \Omega^{2} W(x)\right]_{+} .
$$

The support of $\rho_{\Omega}^{\mathrm{TF}}$ is always compact, but if $\Omega \gtrsim \varepsilon^{-1}$ is sufficiently large, the density vanishes in a neighborhood of the origin. More precisely one can define an analogue, denoted by $\Omega_{\mathrm{c}}$, of the critical value $\omega_{\mathrm{c}}$ as the smallest value of $\Omega$ such that $\rho_{\Omega}^{\mathrm{TF}}$ vanishes at the origin $x=0$. A simple computation shows that

$$
\Omega_{\mathrm{c}}=R_{\mathrm{m}}^{2} R_{\varepsilon}^{-2} \omega_{\mathrm{c}}=2 \sqrt{\frac{2(s+2)}{\pi \gamma(s-2)}}\left(\frac{2}{s}\right)^{\frac{2}{s-2}} \varepsilon^{-1},
$$

i.e., it agrees with what is implied by the scalings (1.9) and (1.15).

If $\Omega \gg \varepsilon^{-1}$ all the expressions simplify and, by using the Taylor expansion

$$
W(x)=\frac{1}{2}(s-2)(1-x)^{2}+\mathcal{O}\left(|1-x|^{3}\right),
$$

and the fact that $\rho_{\Omega}^{\mathrm{TF}}\left(x_{\mathrm{in} / \mathrm{out}}\right)=0$, one obtains

$$
\mu_{\Omega}^{\mathrm{TF}}=\frac{1}{2} \gamma(s-2) \Omega^{2}\left(1-x_{\text {in } / \text { out }}\right)^{2}\left(1+\mathcal{O}\left(\left|1-x_{\text {in } / \text { out }}\right|\right)\right),
$$

so that the normalization condition yields

$$
\left|1-x_{\text {in } / \text { out }}\right|=\left[\frac{3}{2 \pi \gamma(s-2)}\right]^{1 / 3}(\varepsilon \Omega)^{-2 / 3}\left(1+\mathcal{O}\left((\varepsilon \Omega)^{-2 / 3}\right)\right),
$$

and $\mu_{\Omega}^{\mathrm{TF}}=\mathcal{O}\left(\varepsilon^{-4 / 3} \Omega^{2 / 3}\right)$, which in turn implies that

$$
\left\|\rho_{\Omega}^{\mathrm{TF}}\right\|_{L^{\infty}\left(\mathbb{R}^{2}\right)}=\mathcal{O}\left((\varepsilon \Omega)^{2 / 3}\right) .
$$

Note also that for $\Omega \gg \varepsilon^{-1}$ the density can be written as

$$
\rho_{\Omega}^{\mathrm{TF}}(x)=\frac{1}{4} \gamma(s-2) \varepsilon^{2} \Omega^{2}\left[\left(1-x_{\text {in } / \text { out }}\right)^{2}-(1-x)^{2}+\mathcal{O}\left((\varepsilon \Omega)^{-1}\right)\right]_{+} .
$$


For further convenience we introduce another reference density, which is obtained by minimizing a GP-like energy obtained by adding some radial kinetic energy to $\mathcal{E}^{\mathrm{TF}}$, i.e., if $|\log \varepsilon| \ll \omega<\omega_{\mathrm{c}}$,

$$
\hat{\mathcal{E}}_{\omega}^{\mathrm{GP}}[f]:=\int_{\mathbb{R}^{2}} \mathrm{~d} \mathbf{x}\left\{\frac{1}{2}|\nabla f|^{2}-\frac{1}{2} \gamma \omega^{2} x^{2} f^{2}+\varepsilon^{-2}\left[\left(x^{s}+f^{2}\right) f^{2}\right]\right\}
$$

and, if $\Omega_{\mathrm{c}} \leq \Omega \ll \varepsilon^{-4}$,

$$
\hat{\mathcal{E}}_{\Omega}^{\mathrm{GP}}[f]:=\int_{\mathbb{R}^{2}} \mathrm{~d} \mathbf{x}\left\{\frac{1}{2}|\nabla f|^{2}+\gamma \Omega^{2} W(x) f^{2}+\varepsilon^{-2} f^{4}\right\},
$$

where $f \in \hat{\mathscr{D}}^{\mathrm{GP}}$ and

$$
\hat{\mathscr{D}}^{\mathrm{GP}}:=\left\{f \in H^{1}\left(\mathbb{R}^{2}\right), f \geq 0:\|f\|_{2}=1\right\} .
$$

We also set

$$
\hat{E}_{\omega}^{\mathrm{GP}}:=\inf _{f \in \hat{\mathscr{D}}_{\mathrm{GP}}} \hat{\mathcal{E}}_{\omega}^{\mathrm{GP}}[f], \quad \hat{E}_{\Omega}^{\mathrm{GP}}:=\inf _{f \in \hat{\mathscr{D}}_{\mathrm{GP}}} \hat{\mathcal{E}}_{\Omega}^{\mathrm{GP}}[f],
$$

and in both cases we denote (with some abuse of notation) by $g_{0}$ the associated unique nonnegative minimizer.

The energy $\hat{E}_{\omega}^{\mathrm{GP}}$ (resp. $\hat{E}_{\Omega}^{\mathrm{GP}}$ ) is trivially bounded from below by $E_{\omega}^{\mathrm{TF}}$ (resp. $E_{\Omega}^{\mathrm{TF}}$ ). Next proposition proves that it is also bounded from above by the same quantity up to smaller order corrections:

Proposition 2.1 (Estimates for $\hat{E}_{\omega}^{\mathrm{GP}}$ and $\hat{E}_{\Omega}^{\mathrm{GP}}$ ).

If $0 \leq \omega<\omega_{\mathrm{c}}$ as $\varepsilon \rightarrow 0$ then

$$
E_{\omega}^{\mathrm{TF}} \leq \hat{E}_{\omega}^{\mathrm{GP}} \leq E_{\omega}^{\mathrm{TF}}+\mathcal{O}(|\log \varepsilon|)
$$

while, if $\Omega_{\mathrm{c}} \leq \Omega \ll \varepsilon^{-4}$,

$$
E_{\Omega}^{\mathrm{TF}} \leq \hat{E}_{\Omega}^{\mathrm{GP}} \leq E_{\Omega}^{\mathrm{TF}}+\mathcal{O}\left((\varepsilon \Omega)^{4 / 3}\left|\log \left(\varepsilon^{4} \Omega\right)\right|\right)
$$

Remark 2.1 (Radial kinetic energy of $\rho_{\Omega}^{\mathrm{TF}}$ )

Using (2.7) as well as (2.8) and the Taylor expansion (2.5), one easily obtains that

$$
E_{\Omega}^{\mathrm{TF}}=\mathcal{O}\left(\varepsilon^{-4 / 3} \Omega^{2 / 3}\right),
$$

which is much larger than the error term in (2.15), i.e., the radial kinetic energy of the (regularized) TF profile $\rho_{\Omega}^{\mathrm{TF}}$, for any $\Omega \ll \varepsilon^{-4}$, since

$$
\left(\varepsilon^{4} \Omega\right)^{2 / 3}\left|\log \left(\varepsilon^{4} \Omega\right)\right| \ll 1 .
$$

We also remark that employing the variational equation for $g_{0}$ one can show that $\hat{E}_{\Omega}^{\mathrm{GP}} \geq E_{\Omega}^{\mathrm{TF}}+$ $c(\varepsilon \Omega)^{4 / 3}\left|\log \left(\varepsilon^{4} \Omega\right)\right|$ with some $c>0$.

Proof. The result is proven by evaluating the functional $\hat{\mathcal{E}}_{\omega}^{\mathrm{GP}}$ (resp. $\hat{\mathcal{E}}_{\Omega}^{\mathrm{GP}}$ ) on a suitable regularization 11 of $\sqrt{\rho_{\omega}^{\mathrm{TF}}}$ (resp. $\sqrt{\rho_{\Omega}^{\mathrm{TF}}}$ ) like in [CY]. The error terms in (2.14) and (2.15) are precisely given by the kinetic energy of such functions. For instance, if $\Omega \geq \Omega_{\mathrm{c}}$, it is clear that the kinetic energy of the TF profile far from $x_{\text {in/out }}$ is of order $(\varepsilon \Omega)^{4 / 3}$, but one has to regularize the density in a neighborhood of $x_{\text {in }}$ and $x_{\text {out }}$ : By a simple optimization, one can show that the width of such regions has to be of order $\left(\varepsilon^{4} \Omega\right)^{a}(\varepsilon \Omega)^{-2 / 3} \ll\left(x_{\text {out }}-x_{\text {in }}\right)$ for some $a>0$, which produces the additional factor $\left|\log \left(\varepsilon^{4} \Omega\right)\right|$ (see, e.g., CPRY1, Proposition 2.1]).

\footnotetext{
${ }^{11}$ The regularization is necessary since the kinetic energy density is otherwise not integrable around $x=x_{\mathrm{in}} /$ out .
} 
With some slight abuse of notation we denote by $g_{0}$ the minimizer of either (2.10) or (2.11). It has other useful properties which can be easily proven: It is radial and strictly positive and has a unique maximum. Moreover one can show that it is suitably close to $\rho^{\mathrm{TF}}$ : A trivial consequence of (2.14) and (2.15) is (see, e.g., CRY, Proposition 2.1]):

$$
\begin{aligned}
&\left\|g_{0}^{2}-\rho_{\Omega}^{\mathrm{TF}}\right\|_{2}^{2} \leq \mathcal{O}\left(\varepsilon^{2}|\log \varepsilon|\right), \text { if } \quad 0 \leq \omega<\omega_{\mathrm{c}} ; \\
&\left\|g_{0}^{2}-\rho_{\Omega}^{\mathrm{TF}}\right\|_{2}^{2} \leq \mathcal{O}\left(\varepsilon^{10 / 3} \Omega^{4 / 3}\left|\log \left(\varepsilon^{4} \Omega\right)\right|\right), \quad \text { if } \quad \Omega_{\mathrm{c}} \leq \Omega \ll \varepsilon^{-4} .
\end{aligned}
$$

One can also show as in CRY, Proposition 2.1] that

$$
\left\|g_{0}\right\|_{L^{\infty}\left(\mathbb{R}^{2}\right)}^{2} \leq \begin{cases}\left\|\rho_{\omega}^{\mathrm{TF}}\right\|_{\infty}(1+o(1))=\mathcal{O}(1), & \text { if } 0 \leq \omega<\omega_{\mathrm{c}} ; \\ \rho_{\Omega}^{\mathrm{TF}}(1)(1+o(1))=\mathcal{O}\left(\varepsilon^{2 / 3} \Omega^{2 / 3}\right) & \text { if } \Omega_{\mathrm{c}} \leq \Omega \ll \varepsilon^{-4} .\end{cases}
$$

The estimates (2.17) and (2.18) can actually be improved:

\section{Proposition 2.2 (Pointwise estimate for $g_{0}$ ).}

If $0 \leq \omega<\omega \sqrt{12}$ as $\varepsilon \rightarrow 0$, then

$$
\left|g_{0}^{2}(x)-\rho_{\omega}^{\mathrm{TF}}(x)\right| \leq \mathcal{O}\left(|\log \varepsilon|^{-1}\right)
$$

uniformly in $\mathbf{x} \in \mathcal{A}_{\omega}$ with

$$
\mathcal{A}_{\omega}:=\left\{\mathrm{x} \in \mathbb{R}^{2}: 0 \leq x \leq x_{\text {out }}-|\log \varepsilon|^{-1}\right\} .
$$

If on the other hand $\Omega_{\mathrm{c}} \leq \Omega \ll \varepsilon^{-4}$

$$
\left|g_{0}^{2}(x)-\rho_{\Omega}^{\mathrm{TF}}(x)\right| \leq \mathcal{O}\left(\varepsilon^{4 / 3} \Omega^{1 / 3}\left|\log \left(\varepsilon^{4} \Omega\right)\right|^{3 / 2}\right) \rho_{\Omega}^{\mathrm{TF}}(x)=o(1) \rho_{\Omega}^{\mathrm{TF}}(x),
$$

uniformly in $\mathrm{x} \in \mathcal{A}_{\Omega}$ with

$$
\mathcal{A}_{\Omega}:=\left\{\mathbf{x} \in \mathbb{R}^{2}: x_{\text {in }}+(\varepsilon \Omega)^{-2 / 3}\left|\log \left(\varepsilon^{4} \Omega\right)\right|^{-1} \leq x \leq x_{\text {out }}-(\varepsilon \Omega)^{-2 / 3}\left|\log \left(\varepsilon^{4} \Omega\right)\right|^{-1}\right\} .
$$

Remark 2.2 (Bulk of the condensate)

The annulus $\mathcal{A}_{\Omega}$ contains automatically the bulk of the mass provided $\Omega \ll \varepsilon^{-4}$ : Using (2.7) it is simple to verify that

$$
\left\|\rho_{\Omega}^{\mathrm{TF}}\right\|_{L^{1}\left(\mathcal{A}_{\Omega}\right)}=1-\mathcal{O}\left(\left|\log \left(\varepsilon^{4} \Omega\right)\right|^{-2}\right)
$$

and

$$
\rho_{\Omega}^{\mathrm{TF}}(x) \geq C(\varepsilon \Omega)^{2 / 3}\left|\log \left(\varepsilon^{4} \Omega\right)\right|^{-1}>0,
$$

for any $\mathrm{x} \in \mathcal{A}_{\Omega}$. The same obviously holds true for $\mathcal{A}_{\omega}$ as well:

$$
\rho_{\omega}^{\mathrm{TF}}(x) \geq C|\log \varepsilon|^{-1}>0, \quad\left\|\rho_{\omega}^{\mathrm{TF}}\right\|_{L^{1}\left(\mathcal{A}_{\omega}\right)}=1-\mathcal{O}\left(|\log \varepsilon|^{-2}\right) .
$$

As a direct consequence of the pointwise estimates (2.20) and (2.22), $\mathcal{A}_{\omega}$ and $\mathcal{A}_{\Omega}$ contain the bulk of the mass of $g_{0}$ too:

$$
\begin{gathered}
\left\|g_{0}\right\|_{L^{2}\left(\mathcal{A}_{\omega}\right)}^{2}=1-\mathcal{O}\left(|\log \varepsilon|^{-1}\right), \quad \text { if } \quad 0 \leq \omega<\omega_{\mathrm{c}}, \\
\left\|g_{0}\right\|_{L^{2}\left(\mathcal{A}_{\Omega}\right)}^{2}=1-\mathcal{O}\left(\left|\log \left(\varepsilon^{4} \Omega\right)\right|^{-2}\right), \quad \text { if } \quad \Omega_{\mathrm{c}} \leq \Omega \ll \varepsilon^{-4} .
\end{gathered}
$$

\footnotetext{
${ }^{12}$ The condition $\omega<\omega_{\mathrm{c}}$ should be thought of for $\omega=\mathcal{O}\left(\varepsilon^{-1}\right)$ as the stronger requirement $\omega=\omega_{0} \varepsilon^{-1}$ for some constant $\omega_{0}<\omega_{\mathrm{c}} \varepsilon$. Similarly the condition $\Omega \geq \Omega_{\mathrm{c}}$ means actually $\Omega \geq(1-o(1)) \Omega_{\mathrm{c}}$ and, if $\Omega$ is below $\Omega_{\mathrm{c}}, x_{\mathrm{in}}$ has to be set equal to 0 .
} 
Proof. The proof is done exactly as in CRY, Proposition 2.6] (see also CPRY1, Proposition 2.3]), i.e., by identifying local super- and subsolutions to the variational equation solved by $g_{0}$, which can be rewritten

$$
-g_{0}^{\prime \prime}-x^{-1} g_{0}^{\prime}=4 \varepsilon^{-2}\left[\tilde{\rho}-g_{0}^{2}\right] g_{0},
$$

where

$$
\tilde{\rho}(x):= \begin{cases}\frac{1}{2}\left[\varepsilon^{2} \hat{\mu}_{\omega}^{\mathrm{GP}}-x^{s}+\frac{1}{2} \gamma \varepsilon^{2} \omega^{2} x^{2}\right], & \text { if } 0 \leq \omega<\omega_{\mathrm{c}}, \\ \frac{1}{2}\left[\varepsilon^{2} \hat{\mu}_{\Omega}^{\mathrm{GP}}-\gamma \varepsilon^{2} \Omega^{2} W(x)\right], & \text { if } \Omega_{\mathrm{c}} \leq \Omega \ll \varepsilon^{-4},\end{cases}
$$

and the chemical potentials $\hat{\mu}_{\omega}^{\mathrm{GP}}$ and $\hat{\mu}_{\Omega}^{\mathrm{GP}}$ are fixed by the $L^{2}$-normalization of $g_{0}$. Note that $\tilde{\rho}$ differs from the TF densities $\rho_{\omega}^{\mathrm{TF}}$ and $\rho_{\Omega}^{\mathrm{TF}}$ only by the chemical potential. However combining (2.17), (2.18) with (2.19), one can easily obtain the following estimate of the difference between the chemical potentials:

$$
\begin{aligned}
\left|\hat{\mu}_{\omega}^{\mathrm{GP}}-\mu_{\omega}^{\mathrm{TF}}\right| \leq \mathcal{O}\left(\varepsilon^{-1}|\log \varepsilon|^{1 / 2}\right), & \text { if } \quad 0 \leq \omega<\omega_{\mathrm{c}} \\
\left|\hat{\mu}_{\Omega}^{\mathrm{GP}}-\mu_{\Omega}^{\mathrm{TF}}\right| \leq \mathcal{O}\left(\Omega\left|\log \left(\varepsilon^{4} \Omega\right)\right|^{1 / 2}\right), & \text { if } \quad \Omega_{\mathrm{c}} \leq \Omega \ll \varepsilon^{-4} .
\end{aligned}
$$

This in turn yields a pointwise estimate of the difference between $\tilde{\rho}$ and the TF densities, which guarantees that $\tilde{\rho}$ is strictly positive inside $\mathcal{A}_{\omega}$ or $\mathcal{A}_{\Omega}$.

The rest of the proof consists in a local analysis of the variational equation (2.29) on the scales $\varepsilon$ or $\varepsilon^{2 / 3} \Omega^{-1 / 3}\left|\log \left(\varepsilon^{4} \Omega\right)\right|$, if $\omega \leq \omega_{\mathrm{c}}$ or $\Omega_{\mathrm{c}} \leq \Omega \ll \varepsilon^{-4}$ respectively. Note that in this second case the blow-up scale remains much smaller than $(\varepsilon \Omega)^{-2 / 3}\left|\log \left(\varepsilon^{4} \Omega\right)\right|^{-1}$ which appears in the definition of $\mathcal{A}_{\Omega}$.

We conclude this section by stating another pointwise estimate of $g_{0}$ which shows the exponential smallness in $\varepsilon$ of $g_{0}$ outside of $\operatorname{supp}\left(\rho^{\mathrm{TF}}\right)$.

Proposition 2.3 (Exponential smallness of $g_{0}$ ).

There exists a constant $C>0$ such that, if $|\log \varepsilon| \ll \omega$ and $\Omega \ll \varepsilon^{-4}$ as $\varepsilon \rightarrow 0$,

$$
g_{0}^{2}(x) \leq \begin{cases}C \exp \left\{-\varepsilon^{-1 / 2} \operatorname{dist}\left(\mathbf{x}, \operatorname{supp}\left(\rho_{\omega}^{\mathrm{TF}}\right)\right)\right\}, & \text { if }|\log \varepsilon| \ll \omega<\omega_{\mathrm{c}}, \\ C(\varepsilon \Omega)^{2 / 3} \exp \left\{-\Omega^{1 / 2} \operatorname{dist}\left(\mathbf{x}, \operatorname{supp}\left(\rho_{\Omega}^{\mathrm{TF}}\right)\right)\right\}, & \text { if } \Omega_{\mathrm{c}} \leq \Omega \ll \varepsilon^{-4},\end{cases}
$$

for any $\mathbf{x} \in \mathbb{R}^{2}$.

Proof. See, e.g., CRY, Propostion 2.2]: For instance in the first case, i.e., when $|\log \varepsilon| \ll \omega<\omega_{\mathrm{c}}$, one can show that if $x \geq x_{\text {out }}+\sqrt{\varepsilon}$, the r.h.s. of (2.29) is negative and $C \exp \left\{-\varepsilon^{-1 / 2}\left(x-x_{\text {out }}\right)\right\}$ is a supersolution to (2.29) for $x \geq x_{\text {out }}$ and a suitably large constant $C>0$. In the other case when $\Omega_{\mathrm{c}} \leq \Omega \ll \varepsilon^{-4}$ one can investigate (2.29) separately for $x \leq x_{\text {in }}$ and $x \geq x_{\text {out }}$ and identify appropriate supersolutions there.

\subsection{Energy Asymptotics and Emergence of a Hole}

In this section we first prove Theorem 1.1, i.e., the asymptotics of the GP ground state energy, and show how this implies a phase transition at $\Omega_{\mathrm{c}_{2}}$, that is the occurrence of a macroscopic hole in the condensate.

Proof of Theorem 1.1. For the sake of brevity we omit the proof of (1.37) and focus on the regime $\Omega_{\mathrm{c}} \leq \Omega \ll \varepsilon^{-4}$. The strategy as well as most of the estimates are basically the same for (1.37) and (1.38) but the vanishing of the TF density for $x \leq x_{\text {in }}$ makes the second case slightly tougher to deal with.

As usual the result follows from a comparison of appropriate upper and lower bounds to the GP energy $E_{\Omega}^{\mathrm{GP}}$, but in order to simplify the analysis it is convenient to extract the leading order term $E_{\Omega}^{\mathrm{TF}}$ from the outset. This can be done by using a very standard trick (see, e.g., [LM]), i.e., a splitting of the energy: Exploiting the positivity of $g_{0}$, we define a function $u_{0} \in L_{\text {loc }}^{2}\left(\mathbb{R}^{2}\right)$ as

$$
\psi_{\Omega}^{\mathrm{GP}}(\mathbf{x})=: g_{0}(x) u_{0}(\mathbf{x}),
$$


and compute, using the variational equation (2.29) solved by $g_{0}$,

$$
E_{\Omega}^{\mathrm{GP}}=\hat{E}_{\Omega}^{\mathrm{GP}}+\mathcal{F}\left[u_{0}\right]
$$

where $\mathcal{F}$ is the weighted GL-type functional

$$
\mathcal{F}[u]:=\int_{\mathbb{R}^{2}} \mathrm{~d} \mathbf{x} g_{0}^{2}\left\{\frac{1}{2}\left|\left(\nabla-i \mathbf{A}_{\Omega}\right) u\right|^{2}+\varepsilon^{-2} g_{0}^{2}\left(1-|u|^{2}\right)^{2}\right\} .
$$

We can then focus on the reduced energy $\mathcal{F}\left[u_{0}\right]$ and prove upper and lower bounds for it. Note that in the upper bound there is a normalization condition to fulfill, i.e.,

$$
\int_{\mathbb{R}^{2}} \mathrm{~d} \mathbf{x} g_{0}^{2}\left|u_{0}\right|^{2}=1
$$

In the lower bound on the opposite we are going to remove this restriction and minimize w.r.t. any $u \in L^{2}\left(\mathcal{A}_{\Omega}\right)$, where $\mathcal{A}_{\Omega}$ is given by (2.23).

Upper bound: The trial function which is going to be used in the upper bound proof is very similar to the one in [CY, Proof of Proposition 4.1]: Roughly speaking we pick a function $u_{\text {trial }}$ which is approximately a phase factor, i.e., such that $\left|u_{\text {trial }}\right| \simeq 1$, but at the same time contains vortices of unit degree on a regular lattice intersected with the support of the TF density $\rho_{\Omega}^{\mathrm{TF}}$. More precisely we denote by $\mathcal{L}$ a regular lattice with fundamental cell $\mathcal{Q}$ (triangular, square or hexagonal) and lattice spacing $\ell$ and define

$$
u_{\text {trial }}(\mathbf{x}):=c \xi(\mathbf{x}) v(\mathbf{x}),
$$

where $c \simeq 1$ is a normalization constant to enforce (2.37), $v$ a phase factor (here we denote a point $\mathbf{x}=\left(x_{1}, x_{2}\right) \in \mathbb{R}^{2}$ by the complex notation $\left.\zeta=x_{1}+i x_{2}\right)$ given by

$$
v(\mathbf{x}):=\prod_{\zeta_{i} \in \mathcal{L} \cap \mathcal{A}_{\Omega}} \frac{\zeta-\zeta_{i}}{\left|\zeta-\zeta_{i}\right|}=: \exp \{i \varphi(\mathbf{x})\},
$$

and $\xi$ regularizes the singularities of $v$ at the lattice points

$$
\xi(\mathbf{x}):= \begin{cases}1, & \text { if }\left|\mathbf{x}-\mathbf{x}_{i}\right|>t, \text { for all } \mathbf{x}_{i} \in \mathcal{L}, \\ t^{-1}\left|\mathbf{x}-\mathbf{x}_{i}\right|, & \text { if }\left|\mathbf{x}-\mathbf{x}_{i}\right| \leq t\end{cases}
$$

Here $t>0$ is a variational parameter (vortex core radius), such that

$$
t \ll \Omega^{-1 / 2} \text {. }
$$

We also choose the lattice spacing $\ell$ in such a way that

$$
|\mathcal{Q}|=\pi \Omega^{-1},
$$

which guarantees the 'neutrality' of each cell of the lattice in the 'electrostatic analogy' discussed in $\mathrm{CY}$, Proof of Proposition 4.1]. Since $\left|\operatorname{supp}\left(\rho_{\Omega}^{\mathrm{TF}}\right)\right|=\mathcal{O}\left((\varepsilon \Omega)^{-2 / 3}\right)$, the total number of lattice points inside $\operatorname{supp}\left(\rho_{\Omega}^{\mathrm{TF}}\right)$ is $\mathcal{O}\left(\varepsilon^{-2 / 3} \Omega^{-1 / 6}\right) \gg 1$, as long as $\Omega \ll \varepsilon^{-4}$, and it is not difficult to verify that

$$
c=1+\mathcal{O}\left(\varepsilon^{-2 / 3} \Omega^{-1 / 6} t^{2}\right)=1+o(1),
$$

thanks to 2.41).

The evaluation of $\mathcal{F}\left[u_{\text {trial }}\right]$ can be decomposed into two different estimates of the kinetic energy and interaction energy respectively. We are going to show that the former satisfies the inequality

$$
\frac{1}{2} \int_{\mathbb{R}^{2}} \mathrm{~d} \mathbf{x} g_{0}^{2}\left|\left(\nabla-i \mathbf{A}_{\Omega}\right) u_{\text {trial }}\right|^{2} \leq \frac{1}{2}(1+o(1)) \Omega\left|\log \left(t^{2} \Omega\right)\right| .
$$


A simple computation yields (recall that $\xi$ is real)

$$
\begin{array}{r}
\frac{1}{2} \int_{\mathbb{R}^{2}} \mathrm{~d} \mathbf{x} g_{0}^{2}\left|\left(\nabla-i \mathbf{A}_{\Omega}\right) u_{\text {trial }}\right|^{2}=\frac{1}{2} c^{2} \int_{\mathbb{R}^{2}} \mathrm{~d} \mathbf{x} g_{0}^{2} \xi^{2}\left|\nabla \varphi-\mathbf{A}_{\Omega}\right|^{2}+c^{2} \sum_{\mathbf{x}_{i} \in \mathcal{L}} \int_{\left|\mathbf{x}-\mathbf{x}_{i}\right| \leq t} \mathrm{~d} \mathbf{x} g_{0}^{2}|\nabla \xi|^{2} \leq \\
\frac{1}{2}(1+o(1)) \int_{\tilde{\mathcal{A}}} \mathrm{d} \mathbf{x} g_{0}^{2} \xi^{2}\left|\nabla \varphi-\mathbf{A}_{\Omega}\right|^{2}+C \Omega^{1 / 2} t+\mathcal{O}\left(\varepsilon^{\infty}\right),
\end{array}
$$

where $\tilde{\mathcal{A}}$ is chosen in such a way that (2.33) yields the pointwise bound $\sqrt{13} g_{0}=\mathcal{O}\left(\varepsilon^{\infty}\right) \sqrt{14}$ outside $\tilde{\mathcal{A}}$. A possible choice is

$$
\tilde{\mathcal{A}}:=\left\{\mathbf{x} \in \mathbb{R}^{2}: x_{\text {in }}-\Omega^{-1 / 2}\left|\log \left(\varepsilon^{4} \Omega\right)\right|^{2} \leq x \leq x_{\text {out }}+\Omega^{-1 / 2}\left|\log \left(\varepsilon^{4} \Omega\right)\right|^{2}\right\} .
$$

Now we can apply the electrostatic computation in [CY] to estimate the leading term in (2.45): The first term on the r.h.s. of (2.45) becomes

$$
\frac{1}{2} \int_{\tilde{\mathcal{A}}} \mathrm{d} \mathbf{x} g_{0}^{2} \xi^{2}\left|\nabla \varphi-\mathbf{A}_{\Omega}\right|^{2} \leq \frac{1}{2}\left(1+\mathcal{O}\left(t \Omega^{1 / 2}\right)\right) \sum_{\mathbf{x}_{i} \in \mathcal{L} \cap \tilde{\mathcal{A}}^{\mathbf{x} \in \mathcal{Q}^{i}}} \sup _{0}^{2}(x)\left[\pi|\tilde{\mathcal{A}}|\left|\log \left(t^{2} \Omega\right)\right|+\mathcal{O}(1)\right]
$$

as in $\mathrm{CY}$, Eq. (4.37)]. In the above expression we have actually overestimated the contribution in $\tilde{\mathcal{A}} \backslash \mathcal{A}_{\Omega}$ since $v$ contains no vortices there and therefore one would easily get an upper bound without the logarithmic term in the cell contained in $\tilde{\mathcal{A}} \backslash \mathcal{A}_{\Omega}$.

Using a Riemann sum approximation for the integral of $g_{0}^{2}$ in $\tilde{\mathcal{A}}$ we can replace the sum in (2.47) and obtain

$$
\frac{1}{2} \int_{\tilde{\mathcal{A}}} \mathrm{d} \mathbf{x} g_{0}^{2} \xi^{2}\left|\nabla \varphi-\mathbf{A}_{\Omega}\right|^{2} \leq \frac{1}{2}(1+o(1)) \Omega\left[\left|\log \left(t^{2} \Omega\right)\right|+\mathcal{O}(1)\right]
$$

thanks to (2.41) and

$$
\left\|g_{0}\right\|_{L^{2}(\tilde{\mathcal{A}})}^{2} \leq\left\|\rho_{\Omega}^{\mathrm{TF}}\right\|_{L^{1}\left(\mathcal{A}_{\Omega}\right)}+o(1)+\left\|g_{0}\right\|_{L^{2}\left(\tilde{\mathcal{A}} \backslash \mathcal{A}_{\Omega}\right)}^{2} \leq 1+o(1)+\mathcal{O}\left(\left|\log \left(\varepsilon^{4} \Omega\right)\right|^{-2}\right)=1+o(1),
$$

since $g_{0}^{2} \leq(\varepsilon \Omega)^{2 / 3}\left|\log \left(\varepsilon^{4} \Omega\right)\right|^{-1}$ in $\tilde{\mathcal{A}} \backslash \mathcal{A}_{\Omega}$ and $\Omega^{-1 / 2}\left|\log \left(\varepsilon^{4} \Omega\right)\right|^{2} \ll(\varepsilon \Omega)^{-2 / 3}\left|\log \left(\varepsilon^{4} \Omega\right)\right|^{-1}$ for any $\Omega \ll \varepsilon^{-4}$.

The upper bound (2.44) is then proven and it remains to compute the interaction energy of $u_{\text {trial }}$ : For any $\Omega_{\mathrm{c}} \leq \Omega \ll \varepsilon^{-4}$ one can show that

$$
\varepsilon^{-2} \int_{\mathbb{R}^{2}} \mathrm{~d} \mathbf{x} g_{0}^{4}\left(1-\left|u_{\text {trial }}\right|^{2}\right)^{2} \leq C \varepsilon^{-4 / 3} \Omega^{5 / 3} t^{2}
$$

Indeed we note that $\left|u_{\text {trial }}\right|=1$ in $\mathbb{R}^{2} \backslash \mathcal{A}_{\Omega}$, so that

$$
\begin{aligned}
& \int_{\mathbb{R}^{2}} \mathrm{~d} \mathbf{x} g_{0}^{4}\left(1-\left|u_{\text {trial }}\right|^{2}\right)^{2}= \sum_{\mathbf{x}_{i} \in \mathcal{L} \cap \mathcal{A}_{\Omega}} \int_{\mathcal{B}\left(\mathbf{x}_{i}, t\right)} \mathrm{d} \mathbf{x} g_{0}^{4}\left(1-t^{-2}\left|\mathbf{x}-\mathbf{x}_{i}\right|^{2}\right)^{2} \leq \\
& C(\varepsilon \Omega)^{4 / 3} t^{2}|\mathcal{Q}|^{-1}\left|\mathcal{A}_{\Omega}\right| \leq C \varepsilon^{2 / 3} \Omega^{5 / 3} t^{2} .
\end{aligned}
$$

Combining (2.35) with (2.44) and (2.50), we finally obtain

$$
\begin{array}{r}
E_{\Omega}^{\mathrm{GP}} \leq \hat{E}_{\Omega}^{\mathrm{GP}}+\frac{1}{2}(1+o(1)) \Omega\left|\log \left(t^{2} \Omega\right)\right|+C \varepsilon^{-4 / 3} \Omega^{5 / 3} t^{2}+o(1) \leq \\
E_{\Omega}^{\mathrm{TF}}+\frac{1}{6}(1+o(1)) \Omega\left|\log \left(\varepsilon^{4} \Omega\right)\right|+\mathcal{O}(\Omega) .
\end{array}
$$

\footnotetext{
${ }^{13}$ Note that 2.33) also implies an exponential decay in $x$ of $g_{0}$, which is used in order to estimate the remainder in 2.45.

${ }^{14}$ The symbol $\mathcal{O}\left(\varepsilon^{\infty}\right)$ denotes a quantity vanishing faster than any power of $\varepsilon$.
} 
where we have chosen the vortex core radius $t=\varepsilon^{2 / 3} \Omega^{-1 / 3}$ and used (2.15). Note that our choice of $t$ is compatible with (2.41) as long as $\Omega \ll \varepsilon^{-4}$. Moreover the radial kinetic energy of the profile $g_{0}$, which is $\mathcal{O}\left((\varepsilon \Omega)^{4 / 3}\left|\log \left(\varepsilon^{4} \Omega\right)\right|\right)$ according to (2.15), is much smaller than the remainder $\mathcal{O}(\Omega)$ if again $\Omega \ll \varepsilon^{-4}$.

Lower Bound: A lower estimate matching (2.52) can be proven by following the same strategy as in CY, Section 5], i.e., first decomposing the support of $\rho_{\Omega}^{\mathrm{TF}}$ into cells, then blowing-up the energy $\mathcal{F}\left[u_{0}\right]$ on each cell and finally using a lower bound for the GL functional with an applied magnetic field between the second and third critical values (see, e.g., [SS1, SS2]).

The starting point is a restriction of the integration in $\mathcal{F}$ to the domain $\mathcal{A}_{\Omega}$, which exploits the positivity of the integrand. Then we decompose $\mathcal{A}_{\Omega}$ into cells: Let $\tilde{\mathcal{L}}$ be the lattic 15

$$
\tilde{\mathcal{L}}=\left\{\mathbf{x}_{i}=(m \tilde{\ell}, n \tilde{\ell}), m, n \in \mathbb{Z}\right\}
$$

with cells $\tilde{\mathcal{Q}}_{i}$ and lattice spacing satisfying

$$
\Omega^{-1}\left|\log \left(\varepsilon^{4} \Omega\right)\right| \ll \tilde{\ell}^{2} \ll \varepsilon^{-4 / 3} \Omega^{-4 / 3},
$$

we easily obtain the lower bound

$$
\begin{aligned}
\mathcal{F}\left[u_{0}\right] \geq \sum_{\mathbf{x}_{i}, \tilde{\mathcal{Q}}_{i} \subset \mathcal{A}_{\Omega}} \int_{\tilde{\mathcal{Q}}_{i}} \mathrm{~d} \mathbf{x} g_{0}^{2}\left\{\frac{1}{2}\left|\left(\nabla-i \mathbf{A}_{\Omega}\right) u_{0}\right|^{2}+\varepsilon^{-2} g_{0}^{2}\left(1-\left|u_{0}\right|^{2}\right)^{2}\right\} \geq & (1-o(1)) \sum_{\mathbf{x}_{i}, \tilde{\mathcal{Q}}_{i} \subset \mathcal{A}_{\Omega}} g_{0}^{2}\left(x_{i}\right) \mathcal{F}^{(i)}\left[u_{0}\right],
\end{aligned}
$$

where we have used (2.22) and (2.25), which imply the lower bound

$$
g_{0}^{2}(x) \geq C(\varepsilon \Omega)^{2 / 3}\left|\log \left(\varepsilon^{4} \Omega\right)\right|^{-1}, \quad \text { for any } \mathbf{x} \in \mathcal{A}_{\Omega},
$$

and introduced the functional

$$
\mathcal{F}^{(i)}\left[u_{0}\right]:=\int_{\tilde{\mathcal{Q}}_{i}} \mathrm{~d} \mathbf{x}\left\{\frac{1}{2}\left|\left(\nabla-i \mathbf{A}_{\Omega}\right) u_{0}\right|^{2}+\varepsilon^{-4 / 3} \Omega^{2 / 3}\left|\log \left(\varepsilon^{4} \Omega\right)\right|^{-1}\left(1-\left|u_{0}\right|^{2}\right)^{2}\right\} .
$$

Note that the conditions (2.54) on the spacing $\tilde{\ell}$, which are essential for the proof, are compatible as long as $\Omega \ll \varepsilon^{-4}$ : The upper bound on $\tilde{\ell}$ is needed in order that the spacing is much smaller than the width of $\operatorname{supp}\left(\rho_{\Omega}^{\mathrm{TF}}\right)$ as required for the Riemann approximation we shall use. On the other hand, the lower bound in (2.54) implies that the cells of the lattice $\tilde{\mathcal{L}}$ are much larger than those of the lattice considered in the proof of the upper bound. The reason is that we would like each cell of the new lattice $\tilde{\mathcal{L}}$ to contain a large number of vortices, diverging to $\infty$ as $\varepsilon \rightarrow 0$. In fact, this is crucial in order to make the whole proof strategy work since the GL estimate from [SS1, [SS2] that we are going to use holds only under this condition.

We now blow-up the energy in the cell $\tilde{\mathcal{Q}}_{i}$ and set $\mathbf{s}:=\tilde{\ell}^{-1}\left(\mathbf{x}-\mathbf{x}_{i}\right)$,

$$
\tilde{u}_{0}(\mathbf{s}):=u_{0}\left(\mathbf{x}_{i}+\tilde{\ell} \mathbf{x}\right), \quad \tilde{\mathbf{A}}(\mathbf{s}):=\tilde{\ell} \mathbf{A}_{\Omega}\left(\mathbf{x}_{i}+\tilde{\ell} \mathbf{s}\right),
$$

so that $\mathcal{F}^{(i)}$ becomes

$$
\mathcal{F}^{(i)}\left[u_{0}\right]=\tilde{\mathcal{F}}^{(i)}\left[\tilde{u}_{0}\right]:=\int_{\mathcal{Q}_{1}} \mathrm{~d} \mathbf{s}\left\{\frac{1}{2}\left|(\nabla-i \tilde{\mathbf{A}}) \tilde{u}_{0}\right|^{2}+\epsilon^{-2}\left(1-\left|\tilde{u}_{0}\right|^{2}\right)^{2}\right\},
$$

\footnotetext{
${ }^{15}$ For convenience we have chosen a square regular lattice but any regular lattice covering the space would have done the job.
} 
where $\mathcal{Q}_{1}$ stands for a unit square centered at the origin and we have introduced a new small parameter $\epsilon$ defined as

$$
\epsilon:=\tilde{\ell}^{-1} \varepsilon^{2 / 3} \Omega^{-1 / 3}\left|\log \left(\varepsilon^{4} \Omega\right)\right|^{1 / 2},
$$

which is much less than $\left(\varepsilon^{4} \Omega\right)^{1 / 3}\left|\log \left(\varepsilon^{4} \Omega\right)\right|^{1 / 2} \ll 1$ if $\Omega \ll \varepsilon^{-4}$ thanks to (2.54). The rescaled vector potential $\tilde{\mathbf{A}}$ is explicitly given by

$$
\tilde{\mathbf{A}}(\mathbf{s})=\Omega \tilde{\ell} \mathbf{e}_{z} \wedge \mathbf{x}_{i}+\Omega \tilde{\ell}^{2} \mathbf{e}_{z} \wedge \mathbf{s}
$$

and the corresponding magnetic field is

$$
h_{\mathrm{ex}}=\operatorname{curl} \tilde{\mathbf{A}}=\Omega \tilde{\ell}^{2} .
$$

Employing gauge invariance one obtains the lower bound

$$
\tilde{\mathcal{F}}^{(i)}\left[\tilde{u}_{0}\right] \geq \inf _{u \in H^{1}\left(\mathcal{Q}_{1}\right)} \int_{\mathcal{Q}_{1}} \mathrm{~d} \mathbf{s}\left\{\frac{1}{2}\left|\left(\nabla-i h_{\mathrm{ex}} \mathbf{e}_{z} \wedge \mathbf{s}\right) u\right|^{2}+\epsilon^{-2}\left(1-|u|^{2}\right)^{2}\right\},
$$

where the applied magnetic field $h_{\text {ex }}$ satisfies the conditions

$$
|\log \epsilon| \ll h_{\mathrm{ex}} \ll \epsilon^{-2},
$$

thanks to 2.54).

We can now borrow a lower bound to the GL functional with an applied magnetic field between the first and second critical fields (see, e.g., SS1, SS2 ) to estimate the r.h.s. of (2.63) and obtain

$$
\tilde{\mathcal{F}}^{(i)}\left[\tilde{u}_{0}\right] \geq(1-o(1)) h_{\mathrm{ex}} \log \left(\epsilon^{-1} h_{\mathrm{ex}}^{-1 / 2}\right) \geq \frac{1}{6}(1-o(1)) \Omega \tilde{\ell}^{2}\left|\log \left(\varepsilon^{4} \Omega\right)\right|
$$

Putting together the above estimate with (2.55) and using again the Riemann sum approximation we finally get

$$
\mathcal{F}\left[u_{0}\right] \geq \frac{1}{6}(1-o(1)) \Omega\left|\log \left(\varepsilon^{4} \Omega\right)\right|\left\|g_{0}\right\|_{L^{2}\left(\mathcal{A}_{\Omega}\right)}^{2} \geq \frac{1}{6}(1-o(1)) \Omega\left|\log \left(\varepsilon^{4} \Omega\right)\right|,
$$

by (2.28). The splitting (2.35) as well as the trivial bound $\hat{E}_{\Omega}^{\mathrm{GP}} \geq E_{\Omega}^{\mathrm{TF}}$ hence complete the proof of the lower bound.

The occurrence of a hole in the bulk of the condensate for $\Omega>\Omega_{\mathrm{c}}$ now follows as a straightforward consequence of the energy asymptotics proven in Theorem 1.1

Proof of Proposition 1.1. The proof is very similar to the proof of Proposition 2.3 (see also [CRY, Propostion 2.2]): By setting $\rho^{\mathrm{GP}}:=\left|\psi_{\Omega}^{\mathrm{GP}}\right|^{2}$ one easily obtains from (1.25) the inequality

$$
-\frac{1}{2} \Delta \rho^{\mathrm{GP}} \leq 2 \varepsilon^{-2}\left[\tilde{\rho}^{\mathrm{GP}}-\rho^{\mathrm{GP}}\right] \rho^{\mathrm{GP}}
$$

where

$$
\tilde{\rho}^{\mathrm{GP}}(x):=\frac{1}{2}\left[\varepsilon^{2} \mu_{\Omega}^{\mathrm{GP}}-\gamma \varepsilon^{2} \Omega^{2} W(x)\right] .
$$

Now the energy asymptotics (1.38) implies exactly as in the derivation of (2.18) and (2.19) that

$$
\left|\psi_{\Omega}^{\mathrm{GP}}\right| \leq C(\varepsilon \Omega)^{2 / 3}, \quad\left\|\rho^{\mathrm{GP}}-\rho_{\Omega}^{\mathrm{TF}}\right\|_{2}^{2} \leq C \varepsilon^{2} \Omega\left|\log \left(\varepsilon^{4} \Omega\right)\right|,
$$

which in turn (see, e.g., [CRY, Proposition 2.1]) yield the estimate

$$
\left|\mu_{\Omega}^{\mathrm{GP}}-\mu_{\Omega}^{\mathrm{TF}}\right| \leq C \varepsilon^{-2 / 3} \Omega^{5 / 6}\left|\log \left(\varepsilon^{4} \Omega\right)\right|^{1 / 2} .
$$


Now if $\Omega>\Omega_{\mathrm{c}}$, one can pick some $0<\delta \ll 1$ so that the above inequality implies (recall (2.5))

$$
\tilde{\rho}^{\mathrm{GP}}(x) \leq C \varepsilon^{4 / 3} \Omega^{5 / 6}\left|\log \left(\varepsilon^{4} \Omega\right)\right|^{1 / 2}-C(\varepsilon \Omega)^{2 / 3} \delta^{2},
$$

for any $0 \leq x \leq x_{\text {in }}-\delta(\varepsilon \Omega)^{-2 / 3}$. Hence if one takes for instance

$$
\delta=\left(\varepsilon^{4} \Omega\right)^{1 / 12}\left|\log \left(\varepsilon^{4} \Omega\right)\right|
$$

that is $\ll 1$ as long as $\Omega \ll \varepsilon^{-4}$, the inequality (2.67) becomes

$$
-\frac{1}{2} \Delta \rho^{\mathrm{GP}}+C \varepsilon^{-2 / 3} \Omega^{5 / 6} \rho^{\mathrm{GP}} \leq 0, \quad \text { for any } 0 \leq x \leq x_{\mathrm{in}}-\delta(\varepsilon \Omega)^{-2 / 3} .
$$

To complete the proof it suffices therefore to note that the function $C \exp \left\{-\varepsilon^{-1 / 3} \Omega^{5 / 12}\left(x_{\text {in }}-x\right)\right\}$ is a supersolution to the equation $-\Delta f+C \varepsilon^{-2 / 3} \Omega^{5 / 6} f=0$ in the ball $0 \leq x \leq x_{\text {in }}$ with boundary condition $f=C$ on $\partial \mathcal{B}_{x_{\mathrm{in}}}$. One thus obtains

$$
\rho^{\mathrm{GP}}(x) \leq C\left\|\rho^{\mathrm{GP}}\right\|_{\infty} \exp \left\{-\varepsilon^{-1 / 3} \Omega^{5 / 12}\left(x_{\mathrm{in}}-x\right)\right\},
$$

for $0 \leq x \leq x_{\text {in }}$ but, if $\Omega \geq \Omega_{0} \varepsilon^{-1}$ for $\Omega_{0}>\varepsilon \Omega_{\mathrm{c}}$, then $x_{\text {in }}>C>0$ and one can always find some $0<x_{\text {hole }}<x_{\text {in }}$ such that the above upper bound yields the desired estimate.

\subsection{Uniform Distribution of Vorticity}

The energy asymptotics proven in Theorem 1.1 is the main ingredient in the proof of the uniform distribution of vorticity:

Proof of Theorem 1.2. The proof follows very closely [CPRY1, Proof of Theorem 1.1], which in turn relies on [SS1, Proposition 5.1]. For the sake of brevity we only enlighten the major differences and assume that $\Omega_{\mathrm{c}} \leq \Omega \ll \varepsilon^{-4}$.

The starting point is the combination of the cell decomposition (2.55) with the global upper bound (2.52), which gives (recall that $\tilde{\mathcal{L}}$ is a regular square lattice with spacing $\tilde{\ell}$ satisfying (2.54))

$$
\sum_{\mathbf{x}_{i}, \tilde{\mathcal{Q}}_{i} \subset \mathcal{A}_{\Omega}} g_{0}^{2}\left(x_{i}\right) \mathcal{F}^{(i)}\left[u_{0}\right] \leq \frac{1}{6}(1+\eta) \sum_{\mathbf{x}_{i}, \tilde{\mathcal{Q}}_{i} \subset \mathcal{A}_{\Omega}} g_{0}^{2}\left(x_{i}\right) \tilde{\ell}^{2} \Omega\left|\log \left(\varepsilon^{4} \Omega\right)\right|,
$$

for some quantity $\eta:=\eta(\varepsilon, \Omega)$ such that $\eta \rightarrow 0$ as $\varepsilon \rightarrow 0, \Omega \ll \varepsilon^{-4}$.

In order to localize the above bound, one needs some control on the density $g_{0}$ and to this purpose we set

$$
\beta:=\beta(\varepsilon, \Omega)=\max \left(|\log \eta|^{-1},\left|\log \left(\varepsilon^{4} \Omega\right)\right|^{-1}\right),
$$

and define $\mathcal{R}_{\text {bulk }}$ as in (1.42), i.e., $\mathcal{R}_{\text {bulk }}=\left\{\mathbf{x}: x_{\text {in }}+(\varepsilon \Omega)^{-2 / 3} \beta \leq x \leq x_{\text {out }}-(\varepsilon \Omega)^{-2 / 3} \beta\right\}$, so that $\mathcal{R}_{\text {bulk }} \subset \mathcal{A}_{\Omega}$ and

$$
g_{0}^{2}(x) \geq C(\varepsilon \Omega)^{2 / 3} \beta, \quad \text { for any } \mathbf{x} \in \mathcal{R}_{\text {bulk }} .
$$

Such a bound unfortunately is not sufficient to complete the localization of the energy and a certain amount of bad cells has to be rejected: We say that a cell $\tilde{\mathcal{Q}}_{i}$ is good if

$$
\mathcal{F}^{(i)}\left[u_{0}\right] \leq \frac{1}{6}\left[1+\min \left(\sqrt{\eta}, \varepsilon^{2} \sqrt{\Omega}\right)\right] \Omega \tilde{\ell}^{2}\left|\log \left(\varepsilon^{4} \Omega\right)\right| .
$$

Now given any set $\mathcal{S} \subset \mathcal{R}_{\text {bulk }}$ with area much larger than $\tilde{\ell}^{2}$ (2.75) implies that the total number of bad cells contained inside $\mathcal{S}$ (denoted by $N_{B}$ ) is much smaller than the total number of cells $N$, i.e., more precisely

$$
N_{B} \leq \sqrt{\eta} \beta^{-1} N \leq \min \left[\sqrt{\eta}|\log \eta|, \varepsilon^{2} \sqrt{\Omega}\left|\log \left(\varepsilon^{4} \Omega\right)\right|\right] N \ll N .
$$

On the other hand one can use the upper bound (2.78) inside good cells to prove the final result exactly as in CPRY1, Proof of Theorem 1.1]. Note that the choice $\tilde{\ell}=\Omega^{-1 / 2}\left|\log \left(\varepsilon^{4} \Omega\right)\right|$ is compatible with (2.54) and yields the lower bound on the measure of $\mathcal{S}$, i.e., $|\mathcal{S}| \gg \tilde{\ell}^{2}=\Omega^{-1}\left|\log \left(\varepsilon^{4} \Omega\right)\right|^{2}$. 


\section{The Giant Vortex Regime $\Omega \sim \varepsilon^{-4}$}

Since throughout this section we deal only with the regime $\Omega \sim \varepsilon^{-4}$, we remove any suffix from $\psi_{\Omega}^{\mathrm{GP}}$, $E_{\Omega}^{\mathrm{GP}}$, etc. for the sake of simplicity and denote those quantities by $\psi^{\mathrm{GP}}, E^{\mathrm{GP}}$ etc..

\subsection{Preliminary Estimates for $E^{\mathrm{GP}}$ and $\psi^{\mathrm{GP}}$}

We first introduce and recall some notation: As in (1.53) we define a parameter $\alpha$ as

$$
\alpha^{2}:=4+\gamma(s-2)
$$

and a vector field $\mathbf{B}$ by

$$
\mathbf{B}=\left(\Omega r-\lfloor\Omega\rfloor r^{-1}\right) \mathbf{e}_{\vartheta} .
$$

A Taylor expansion of the potential $U(x)$ (see (1.50) ) around $x=1$ (e.g., for $\frac{1}{2} \leq x \leq \frac{3}{2}$ ) yields

$$
\begin{array}{r}
U(x)=\frac{1}{2} B^{2}(1)+\left(1-\lfloor\Omega\rfloor^{2} \Omega^{-2}\right)(x-1)+\frac{1}{2}\left[1+3\lfloor\Omega\rfloor^{2} \Omega^{-2}+\gamma(s-2)\right](x-1)^{2}+\mathcal{O}\left((x-1)^{3}\right)= \\
\frac{1}{2} \alpha^{2}(x-1)^{2}+\mathcal{O}\left(\Omega^{-2}\right)+\mathcal{O}\left((x-1)^{3}\right) .
\end{array}
$$

Moreover we denote by $g_{\text {osc }}$ the normalized ground state of the one-dimensional harmonic oscillator $(y \in \mathbb{R})$

$$
H_{\mathrm{osc}}:=-\frac{1}{2} \partial_{y}^{2}+\frac{1}{2} \alpha^{2} y^{2}
$$

i.e.,

$$
g_{\text {osc }}(y):=\pi^{-1 / 4} \alpha^{1 / 4} \exp \left\{-\frac{1}{2} \alpha y^{2}\right\} .
$$

The starting point is a rough but useful upper bound on $E^{\mathrm{GP}}$ which has several important consequences:

Proposition 3.1 (Estimates for $E^{\mathrm{GP}}$ and $\psi^{\mathrm{GP}}$ ). If $\Omega=\Omega_{0} \varepsilon^{-4}$ with $\Omega_{0}>0$ and $\varepsilon$ is sufficiently small,

$$
E^{\mathrm{GP}} \leq \mathcal{O}(\Omega), \quad\left\|\psi^{\mathrm{GP}}\right\|_{\infty}^{2} \leq \mathcal{O}\left(\varepsilon^{2} \Omega\right)
$$

Moreover there exists a finite constant $C$ such that, for any $\mathbf{x} \in \mathbb{R}^{2}$,

$$
\left|\psi^{\mathrm{GP}}(\mathbf{x})\right|^{2} \leq C \varepsilon^{-2} \exp \left\{-\Omega^{1 / 2}|1-x|\right\}
$$

Proof. In order to prove the upper bound on $E^{\mathrm{GP}}$, it suffices to test the GP functional on a suitable rescaling of the harmonic oscillator ground state (3.4) times a phase containing a giant vortex of degree $\lfloor\Omega\rfloor$ at the origin. More precisely we set

$$
\begin{gathered}
\psi_{\text {trial }}(\mathbf{x}):=f_{\text {trial }}(x) e^{i\lfloor\Omega\rfloor \vartheta}, \\
f_{\text {trial }}(x):=c_{\Omega} \begin{cases}\Omega^{1 / 4} g_{\text {osc }}\left(\Omega^{1 / 2}(1-x)\right), & \text { if } x \geq 1-\varepsilon, \\
f(x), & \text { if } 1-2 \varepsilon \leq x \leq 1-\varepsilon, \\
0, & \text { if } 0 \leq x \leq 1-2 \varepsilon,\end{cases}
\end{gathered}
$$

where $c_{\Omega}$ is a normalization constant and $f$ a smooth function such that

$$
f(1-2 \varepsilon)=0, \quad f(1-\varepsilon)=g_{\mathrm{osc}}\left(\Omega^{1 / 2} \varepsilon\right)=\mathcal{O}\left(\varepsilon^{\infty}\right),
$$


which also allows to assume that $\|\nabla f\|_{\infty}=\mathcal{O}\left(\varepsilon^{\infty}\right)$. A simple computation gives

$$
\begin{aligned}
& 1=\left\|\psi_{\text {trial }}\right\|_{2}^{2}=2 \pi c_{\Omega}^{2} \Omega^{1 / 2} \int_{1-\varepsilon}^{\infty} \mathrm{d} x x g_{\text {osc }}^{2}\left(\Omega^{1 / 2}(x-1)\right)+\mathcal{O}\left(\varepsilon^{\infty}\right)= \\
& 2 \pi c_{\Omega}^{2} \int_{-\varepsilon \Omega^{1 / 2}}^{\infty} \mathrm{d} y\left(1+\Omega^{-1 / 2} y\right) g_{\text {osc }}^{2}(y)+\mathcal{O}\left(\varepsilon^{\infty}\right)=2 \pi c_{\Omega}^{2} \int_{-\infty}^{\infty} \mathrm{d} y g_{\text {osc }}^{2}(y)+\mathcal{O}\left(\Omega^{-1 / 2}\right),
\end{aligned}
$$

so that

$$
c_{\Omega}^{2}=(2 \pi)^{-1}\left(1+\mathcal{O}\left(\Omega^{-1 / 2}\right)\right) .
$$

By using (3.2) and the exponential decay of $g_{\text {osc }}$ one has

$$
\begin{gathered}
\mathcal{E}_{\Omega}^{\mathrm{GP}}\left[\psi_{\text {trial }}\right]=2 \pi c_{\Omega}^{2} \int_{0}^{\infty} \mathrm{d} x x\left\{\frac{1}{2}\left(f_{\text {trial }}^{\prime}\right)^{2}+\Omega^{2} U(x) f_{\text {trial }}^{2}+c_{\Omega}^{2} \varepsilon^{-2} f_{\text {trial }}^{4}\right\}= \\
2 \pi c_{\Omega}^{2} \int_{1-\varepsilon}^{1+\varepsilon} \mathrm{d} x x\left\{\frac{1}{2}\left(f_{\text {trial }}^{\prime}\right)^{2}+\Omega^{2} U(x) f_{\text {trial }}^{2}+c_{\Omega}^{2} \varepsilon^{-2} f_{\text {trial }}^{4}\right\}+\mathcal{O}\left(\varepsilon^{\infty}\right)= \\
2 \pi c_{\Omega}^{2} \Omega \int_{-\varepsilon \Omega^{1 / 2}}^{\varepsilon \Omega^{1 / 2}} \mathrm{~d} y\left(1+\Omega^{-1 / 2} y\right)\left\{\frac{1}{2}\left(g_{\text {osc }}^{\prime}\right)^{2}+\frac{1}{2} \alpha^{2} y^{2} g_{\text {osc }}^{2}+c_{\Omega}^{2} \varepsilon^{-2} \Omega^{-1 / 2} g_{\text {osc }}^{4}\right\}+\mathcal{O}\left(\Omega^{-1}\right)+\mathcal{O}\left(\Omega \varepsilon^{3}\right)= \\
2 \pi c_{\Omega}^{2} \Omega\left[\frac{\alpha}{2}+c_{\Omega}^{2} \sqrt{\frac{\alpha}{2 \pi \Omega_{0}}}+\mathcal{O}\left(\Omega^{-1 / 2}\right)\right]=\Omega\left[\frac{\alpha}{2}+\frac{1}{2 \pi} \sqrt{\frac{\alpha}{2 \pi \Omega_{0}}}+\mathcal{O}\left(\Omega^{-1 / 2}\right)\right] .
\end{gathered}
$$

The second statement in (3.5) is a consequence of the variational equation (1.25) which yields

$$
-\frac{1}{2} \Delta \rho^{\mathrm{GP}} \leq \varepsilon^{-2}\left[\varepsilon^{2} \mu^{\mathrm{GP}}-\gamma \varepsilon^{2} \Omega^{2} W-2 \rho^{\mathrm{GP}}\right] \rho^{\mathrm{GP}} \leq \varepsilon^{-2}\left[\varepsilon^{2} \mu^{\mathrm{GP}}-2 \rho^{\mathrm{GP}}\right] \rho^{\mathrm{GP}},
$$

where

$$
\rho^{\mathrm{GP}}:=\left|\psi^{\mathrm{GP}}\right|^{2}
$$

and we have used the positivity of $W$. Now since $\Delta \rho^{\mathrm{GP}} \leq 0$ at any maximum point of $\rho^{\mathrm{GP}}$, it immediately follows that

$$
\left\|\rho^{\mathrm{GP}}\right\|_{\infty} \leq \frac{1}{2} \varepsilon^{2} \mu^{\mathrm{GP}}
$$

which together with the definition of the chemical potential implies

$$
\mu^{\mathrm{GP}}=E^{\mathrm{GP}}+\varepsilon^{-2}\left\|\psi^{\mathrm{GP}}\right\|_{4}^{4} \leq E^{\mathrm{GP}}+\frac{1}{2} \mu^{\mathrm{GP}},
$$

so that by (3.10)

$$
\mu^{\mathrm{GP}} \leq 2 E^{\mathrm{GP}} \leq \mathcal{O}(\Omega)
$$

and thus (3.5) is proven.

In order to prove the pointwise estimate (3.6) we exhibit an explicit supersolution to the variational equation (1.25). We first consider a ball $\mathcal{B}\left(1-a \varepsilon^{2}\right)$ centered at the origin of radius $1-a \varepsilon^{2}$, with $a=\mathcal{O}(1)$ sufficiently large: The monotonicity of $W$ together with the Taylor expansion $W(x)=\frac{1}{2}(s-2)(1-x)^{2}+$ $\mathcal{O}\left((1-x)^{3}\right)$, yields the bound

$$
W(x) \geq C a^{2} \varepsilon^{4},
$$

for some $C>0$ and for any $0 \leq x \leq 1-a \varepsilon^{2}$. Therefore inside $\mathcal{B}\left(1-a \varepsilon^{2}\right)$ one has by (3.11) and (3.14)

$$
-\frac{1}{2} \Delta \rho^{\mathrm{GP}} \leq \varepsilon^{-2}\left[\varepsilon^{2} \mu^{\mathrm{GP}}-C a^{2} \varepsilon^{6} \Omega^{2}\right] \rho^{\mathrm{GP}} \leq-\frac{1}{2} C a^{2} \varepsilon^{4} \Omega^{2} \rho^{\mathrm{GP}},
$$

if $a$ is taken sufficiently large. In the same region the function

$$
f_{\text {sup }}(x):=C_{a}\left\|\rho^{\mathrm{GP}}\right\|_{\infty} \exp \left\{-\Omega^{1 / 2}(1-x)\right\}
$$


satisfies

$$
-\frac{1}{2} \Delta f_{\text {sup }}+\frac{1}{2} C a^{2} \varepsilon^{4} \Omega^{2} f_{\text {sup }}=\frac{1}{2}\left[-\Omega^{1 / 2}-\Omega x+C a^{2} \varepsilon^{4} \Omega^{2}\right] f_{\text {sup }}>0,
$$

if $a$ is again large enough. Therefore $f_{\text {sup }}$ provides a supersolution, since at the boundary $\partial \mathcal{B}\left(1-a \varepsilon^{2}\right)$ one has

$$
f_{\text {sup }}\left(1-a \varepsilon^{2}\right)=C_{a}\left\|\rho^{\mathrm{GP}}\right\|_{\infty} \exp \left\{-a \Omega_{0}^{1 / 2}\right\}=\left\|\rho^{\mathrm{GP}}\right\|_{\infty}
$$

if $C_{a}$ is taken equal to $\exp \left\{a \Omega_{0}^{1 / 2}\right\}$. In conclusion, by the maximum principle (see, e.g., $\mathrm{T}$, Exercise 2, p. 317] or [E, Theorem 1, p. 508]) $\rho^{\mathrm{GP}}(x) \leq f_{\text {sup }}(x)$ for any $\mathbf{x} \in \mathcal{B}_{1-a \varepsilon^{2}}$ but the result can be trivially extended to the whole ball $\mathcal{B}_{1}$ thanks to the monotonicity of $f_{\text {sup }}$.

To complete the proof of the pointwise estimate (3.6), one has simply to repeat the argument above in the complement of the region $\mathcal{B}_{1+a \varepsilon^{2}}$, exploiting the monotonicity of $W$ there, and replace the supersolution $f_{\text {sup }}$ with the function

$$
C_{a}\left\|\rho^{\mathrm{GP}}\right\|_{\infty} \exp \left\{-\Omega^{1 / 2}(x-1)\right\} .
$$

The decay of $\psi^{\mathrm{GP}}$ outside of the bulk of the condensate can be estimated more precisely by exploiting the Taylor expansion (2.5):

Proposition 3.2 (Exponential decay of $\left.\psi^{\mathrm{GP}}\right)$.

If $\Omega=\Omega_{0} \varepsilon^{-4}$ with $\Omega_{0}>0$ and $1 \ll \lambda \ll|\log \varepsilon|^{b}$ for some $b>0$ as $\varepsilon \rightarrow 0$, there exist two finite constants $c>0$ and $C<\infty$ independent of $\varepsilon$ and $\lambda$ such that

$$
\left|\psi^{\mathrm{GP}}(\mathbf{x})\right|^{2} \leq C \varepsilon^{-2} \exp \left\{-c \Omega\left(|1-x|-\varepsilon^{2} \lambda\right)^{2}\right\},
$$

for any $\mathbf{x}$ satisfying the condition

$$
\varepsilon^{2} \lambda \leq|1-x| \leq \varepsilon^{2} \lambda^{a}, \quad \text { with } a \geq 2 .
$$

Proof. We first consider the region $x \leq 1-\varepsilon^{2} \lambda$ and prove that the function

$$
f_{\text {sup }}(x):=\left\|\rho^{\mathrm{GP}}\right\|_{\infty}\left[\exp \left\{-c \Omega\left(1-\varepsilon^{2} \lambda-x\right)^{2}\right\}+\exp \left\{-\sqrt{\Omega_{0}} \lambda^{2 a+1}\right\}\right]
$$

provides a supersolution to (3.11) in the region $\varepsilon^{2} \lambda \leq 1-x \leq \varepsilon^{2} \lambda^{2 a+1}$, for any $a \geq 2$ :

$$
\begin{aligned}
& {\left[-\frac{1}{2} \Delta+\gamma \Omega^{2} W(x)+2 \varepsilon^{-2} f_{\text {sup }}^{2}(x)-\mu^{\mathrm{GP}}\right] f_{\text {sup }} \geq} \\
& \quad\left[\frac{1}{2} c \Omega+\frac{1}{2}\left(s-2-c^{2}\right) \Omega^{2}(1-x)^{2}-C \varepsilon^{-2} \lambda^{6 a+3}-C \Omega\right] f_{\text {sup }} \geq \\
& \quad \varepsilon^{-4}\left[\frac{1}{2}\left(s-2-c^{2}\right) \Omega_{0}^{2} \lambda^{2}-C \Omega_{0}\right] f_{\text {sup }} \geq 0
\end{aligned}
$$

if $c^{2}<s-2$ and $\varepsilon$ is sufficiently small (recall that by assumption $\lambda \rightarrow \infty$ as $\varepsilon \rightarrow 0$ ). Moreover at the boundary

$$
f_{\text {sup }}\left(1-\varepsilon^{2} \lambda\right) \geq\left\|\rho^{\mathrm{GP}}\right\|_{\infty} \geq \rho^{\mathrm{GP}}\left(1-\varepsilon^{2} \lambda\right), \quad f_{\text {sup }}\left(1-\varepsilon^{2} \lambda^{2 a+1}\right) \geq \rho^{\mathrm{GP}}\left(1-\varepsilon^{2} \lambda^{2 a+1}\right),
$$

thanks to (3.6). Therefore $\rho^{\mathrm{GP}} \leq f_{\text {sup }}$ and in the region (3.17)

$$
\exp \left\{c \Omega\left(1-\varepsilon^{2} \lambda-x\right)^{2}-\sqrt{\Omega_{0}} \lambda^{2 a+1}\right\} \leq \exp \left\{-\sqrt{\Omega_{0}} \lambda^{2 a}(\lambda-C)\right\}=o(1) .
$$

The proof for $x \geq 1+\varepsilon^{2} \lambda$ is identical. 


\subsection{The Giant Vortex Density Profile}

In this section we investigate the properties of the giant vortex profile and the associated energy functional defined in (1.49).

Proposition 3.3 (Minimization of $\mathcal{E}^{\mathrm{gv}}$ ).

There exists a minimizer $g_{\mathrm{gv}}$ of (1.49) that is unique up to a sign, radial and can be chosen to be strictly positive for $\mathbf{x} \neq 0$. It solves the variational equation

$$
-\frac{1}{2} \Delta g_{\mathrm{gv}}+\Omega^{2} U(x) g_{\mathrm{gv}}+2 \varepsilon^{-2} g_{\mathrm{gv}}^{3}=\mu^{\mathrm{gv}} g_{\mathrm{gv}},
$$

with $\mu^{\mathrm{gv}}=E^{\mathrm{gv}}+\varepsilon^{-2}\left\|g_{\mathrm{gv}}\right\|_{4}^{4}$. Moreover $g_{\mathrm{gv}}$ has a unique maximum and, if $\Omega=\Omega_{0} \varepsilon^{-4}$ with $\Omega_{0}>0$,

$$
E^{\mathrm{gv}}=\Omega\left[\frac{\alpha}{2}+\frac{1}{2 \pi} \sqrt{\frac{\alpha}{2 \pi \Omega_{0}}}+\mathcal{O}\left(\Omega_{0}^{-3 / 4}\right)+\mathcal{O}\left(\Omega^{-1 / 2}\right)\right] .
$$

In addition there exists a finite constant $C$ such that

$$
\sup _{x \in \mathbb{R}^{2}}\left|\sqrt{2 \pi} \Omega^{-1 / 4} g_{\mathrm{gv}}(x)-g_{\mathrm{osc}}\left(\Omega^{1 / 2}(1-x)\right)\right| \leq C \Omega_{0}^{-1 / 4} \varepsilon^{-1}
$$

Finally $g_{\mathrm{gv}}$ decays exponentially far from $x=1$ : If $1 \ll \lambda \ll|\log \varepsilon|^{b}$ for some $b>0$ there exist constants $c>0$ and $C<\infty$ independent of $\varepsilon$ and $\lambda$ such that, for any $\varepsilon^{2} \lambda \leq|1-x| \leq \varepsilon^{2} \lambda^{a}, a \geq 2$,

$$
g_{\mathrm{gv}}^{2}(x) \leq C \varepsilon^{-2} \exp \left\{-c \Omega\left(|1-x|-\varepsilon^{2} \lambda\right)^{2}\right\}
$$

Proof. All the properties of $g_{\mathrm{gv}}$ can be deduced by standard arguments (see, e.g., [CRY, Proposition 2.3] or [CPRY1, Proposition 4.1]). For instance the existence of a unique maximum point of $g_{\mathrm{gv}}$ can be proven by an adaptation of the argument in CPRY1, Proposition 2.2], while the pointwise estimate (3.24) can be proven exactly as (3.16).

The energy upper bound in (3.22), i.e.,

$$
E^{\mathrm{gv}} \leq \Omega\left[\frac{\alpha}{2}+\frac{1}{2 \pi} \sqrt{\frac{\alpha}{2 \pi \Omega_{0}}}+\mathcal{O}\left(\Omega^{-1 / 2}\right)\right]
$$

has already been proven in (3.10). In order to prove a lower bound matching with (3.10), one needs first to show that

$$
\left\|g_{\mathrm{gv}}(x)-(2 \pi)^{-1 / 2} \Omega^{1 / 4} g_{\mathrm{osc}}\left(\Omega^{1 / 2}(1-x)\right)\right\|_{L^{2}\left(\mathbb{R}^{2}\right)}=\mathcal{O}\left(\Omega_{0}^{-1 / 4}\right)
$$

After a rescaling of all lengths in the functional (1.49), i.e., setting $y:=\Omega^{1 / 2}(1-x)$, and denoting

$$
\tilde{g}_{\mathrm{gv}}(y):=(2 \pi)^{1 / 2} \Omega^{-1 / 4} g_{\mathrm{gv}}\left(1-\Omega^{-1 / 2} y\right)
$$

one obtains

$$
\begin{aligned}
& E^{\mathrm{gv}}=\mathcal{E}^{\mathrm{gv}}\left[g_{\mathrm{gv}}\right] \geq \Omega\left(1-\mathcal{O}\left(\Omega^{-1 / 2}\right)\right) \int_{-\infty}^{\frac{1}{2} \Omega^{1 / 2}} \mathrm{~d} y\left\{\frac{1}{2}\left|\nabla \tilde{g}_{\mathrm{gv}}\right|^{2}+\frac{1}{2} \alpha^{2} y^{2} \tilde{g}_{\mathrm{gv}}^{2}+(2 \pi)^{-1} \Omega_{0}^{-1 / 2} \tilde{g}_{\mathrm{gv}}^{4}\right\} \geq \\
& \Omega\left(1-\mathcal{O}\left(\Omega^{-1 / 2}\right)\right)\left\langle\tilde{g}_{\mathrm{gv}}\left|H_{\mathrm{osc}}\right| \tilde{g}_{\mathrm{gv}}\right\rangle
\end{aligned}
$$

where we have used the Taylor expansion (3.2) and $H_{\mathrm{osc}}$ is defined in (3.3). Strictly speaking the expectation value on the r.h.s. of the above expressions is computed in $L^{2}\left(-\infty, \frac{1}{2} \Omega^{1 / 2}\right)$ but, exploiting 
the exponential smallness (3.24) of $g_{\mathrm{gv}}$ at $y=\frac{1}{2} \Omega^{1 / 2}$, it is not difficult to slightly extend $\tilde{g}_{\mathrm{gv}}$ smoothly to $y$ larger than $\frac{1}{2} \Omega^{1 / 2}$ in such a way that the mean value can be computed in $L^{2}(\mathbb{R})$. The error $\mathcal{O}\left(\varepsilon^{\infty}\right)$ due to this procedure can safely be included in the remainder $\mathcal{O}\left(\Omega_{0}^{-1 / 2}\right)$.

We now decompose $\tilde{g}_{\mathrm{gv}}$ in a Fourier series in terms of the normalized eigenfunctions $g_{n}(y), n \in \mathbb{N}$, of the harmonic oscillator (3.3) (with $g_{0}=g_{\text {osc }}$ ), i.e., set

$$
\tilde{g}_{\mathrm{gv}}(y)=\sum_{n=0}^{\infty} a_{n} g_{n}(y)
$$

and use the upper bounds above to get

$$
\frac{\alpha\left|a_{0}\right|^{2}}{2}+\frac{3 \alpha}{2} \sum_{n=1}^{\infty}\left|a_{n}\right|^{2} \leq \alpha \sum_{n=0}^{\infty}\left(n+\frac{1}{2}\right)\left|a_{n}\right|^{2}=\left\langle\tilde{g}_{\mathrm{gv}}\left|H_{\mathrm{osc}}\right| \tilde{g}_{\mathrm{gv}}\right\rangle \leq \frac{\alpha}{2}+\frac{1}{2 \pi} \sqrt{\frac{\alpha}{2 \pi \Omega_{0}}}+\mathcal{O}\left(\Omega^{-1 / 2}\right),
$$

which immediately implies

$$
\sum_{n=1}^{\infty}\left|a_{n}\right|^{2} \leq C \Omega_{0}^{-1 / 2}
$$

since $\sum\left|a_{n}\right|^{2}=1-\mathcal{O}\left(\varepsilon^{\infty}\right)$ by (3.24). Therefore (3.26) is proven and, since $\left\|\tilde{g}_{\mathrm{gv}}\right\|_{\infty} \leq C \Omega_{0}^{1 / 2}$ (see below), one also has

$$
\left\|\tilde{g}_{\mathrm{gv}}\right\|_{4}^{4} \geq\left\|g_{\mathrm{osc}}\right\|_{4}^{4}-C \Omega_{0}^{1 / 2}\left\|\tilde{g}_{\mathrm{gv}}-g_{\mathrm{osc}}\right\|_{2}-\mathcal{O}\left(\varepsilon^{\infty}\right) \geq\left\|g_{\mathrm{osc}}\right\|_{4}^{4}-C \Omega_{0}^{1 / 4}
$$

which yields (3.22)

In fact (3.31) has much stronger consequences than (3.26) and, if we define for any $g \in L^{2}(\mathbb{R})$

$$
\|g\|_{\mathrm{osc}}^{2}:=\left\langle g\left|H_{\mathrm{osc}}\right| g\right\rangle,
$$

one has

$$
\left\|\tilde{g}_{\mathrm{gv}}-g_{\mathrm{osc}}\right\|_{\mathrm{osc}}^{2}=\left\langle\tilde{g}_{\mathrm{gv}}\left|H_{\mathrm{osc}}\right| \tilde{g}_{\mathrm{gv}}\right\rangle-\alpha\left\langle\tilde{g}_{\mathrm{gv}} \mid g_{\mathrm{osc}}\right\rangle+\frac{1}{2} \alpha \leq C \Omega_{0}^{-1 / 2},
$$

where we have used (3.22) and the inequality

$$
\left\langle\tilde{g}_{\mathrm{gv}} \mid g_{\mathrm{osc}}\right\rangle \geq 1-C \Omega_{0}^{-1 / 2},
$$

which is a trivial consequence of (3.26). On the other hand the Sobolev embedding theorem in $\mathbb{R}$ immediately implies

$$
\|g\|_{L^{\infty}(\mathbb{R})} \leq C\|g\|_{H^{1}(\mathbb{R})} \leq C\|g\|_{\text {osc }},
$$

and therefore (3.23) follows from (3.34). Note that the prefactor in (3.23) is bounded independently of $\Omega_{0}$ because it behaves as $\Omega_{0}^{-1 / 4} \Omega^{1 / 4}$.

For technical reasons which will be clearer later we also consider a functional with a different integration domain, i.e.,

$$
\mathcal{A}_{\eta_{\varepsilon}}:=\left\{\mathbf{x} \in \mathbb{R}^{2}: 1-\varepsilon^{2} \eta_{\varepsilon} \leq x \leq 1+\varepsilon^{2} \eta_{\varepsilon}\right\},
$$

where

$$
|\log \varepsilon| \ll \eta_{\varepsilon} \ll|\log \varepsilon|^{b}
$$

with $b>1$ is a parameter which will be fixed later as $\eta_{\varepsilon}=|\log \varepsilon|^{3 / 2}$. Note that since $\eta_{\varepsilon} \gg 1$ the domain $\mathcal{A}_{\eta_{\varepsilon}}$ expands on a scale $\varepsilon^{-2}$, which ensures that it contains the bulk of the mass. We define

$$
\mathscr{D}_{\eta_{\varepsilon}}^{g v}:=\left\{f \in H^{1}\left(\mathcal{A}_{\eta_{\varepsilon}}\right): f=f^{*},\|f\|_{L^{2}\left(\mathcal{A}_{\eta_{\varepsilon}}\right)}=1\right\}
$$


and set, for any $f \in \mathscr{D}_{\eta_{\varepsilon}}^{\mathrm{gv}}$,

$$
\mathcal{E}_{\eta_{\varepsilon}}^{\mathrm{gv}}[f]:=\int_{\mathcal{A}_{\eta_{\varepsilon}}} \mathrm{d} \mathbf{x}\left\{\frac{1}{2}|\nabla f|^{2}+\Omega^{2} U(x) f^{2}+\varepsilon^{-2} f^{4}\right\} .
$$

We recall that

$$
U(x)=\frac{1}{2} B^{2}(x)+\gamma W(x)=\frac{1}{2}\lfloor\Omega\rfloor^{2} \Omega^{-2} x^{-2}+\frac{1}{2} x^{2}+\frac{1}{s} \gamma\left(x^{s}-1\right)-\frac{1}{2} \gamma\left(x^{2}-1\right)-\lfloor\Omega\rfloor \Omega^{-1} \geq 0 .
$$

The ground state energy is

$$
E_{\eta_{\varepsilon}}^{\mathrm{gv}}:=\inf _{f \in \mathscr{D}_{\eta_{\varepsilon}}^{\mathrm{gv}}} \mathcal{E}_{\eta_{\varepsilon}}^{\mathrm{gv}}[f]
$$

and we denote by $g_{\eta_{\varepsilon}}$ any associated minimizer.

Proposition 3.4 (Minimization of $\mathcal{E}_{\eta_{\varepsilon}}^{\mathrm{gv}}$ ).

There exists a minimizer $g_{\eta_{\varepsilon}}$ of (3.39) that is unique up to a sign, radial and can be chosen to be strictly positive for $\mathbf{x} \neq 0$. Inside $\mathcal{A}_{\eta_{\varepsilon}}$ it solves the variational equation

$$
-\frac{1}{2} \Delta g_{\eta_{\varepsilon}}+\Omega^{2} U(x) g_{\eta_{\varepsilon}}+2 \varepsilon^{-2} g_{\eta_{\varepsilon}}^{3}=\mu_{\eta_{\varepsilon}}^{\mathrm{gv}} g_{\eta_{\varepsilon}},
$$

with boundary conditions $g_{\eta_{\varepsilon}}^{\prime}\left(1 \pm \varepsilon^{2} \eta_{\varepsilon}\right)=0$ and $\mu_{\eta_{\varepsilon}}^{\mathrm{gv}}=E_{\eta_{\varepsilon}}^{\mathrm{gv}}+\varepsilon^{-2}\left\|g_{\eta_{\varepsilon}}\right\|_{4}^{4}$. Moreover $g_{\eta_{\varepsilon}}$ has a unique global maximum at $x_{\max }$. If $\Omega=\Omega_{0} \varepsilon^{-4}$ with $\Omega_{0}>0$, then $\left\|g_{\eta_{\varepsilon}}\right\|_{\infty}^{2}=\mathcal{O}\left(\varepsilon^{2} \Omega\right)$ and

$$
E_{\eta_{\varepsilon}}^{\mathrm{gv}}=\left(1+\mathcal{O}\left(\varepsilon^{\infty}\right)\right) E^{\mathrm{gv}} .
$$

Proof. The only result which deserves a discussion is the energy upper bound (3.41) since anything else can be dealt with as in the proof of Propositions 3.1 and 3.3 However it suffices to test the functional $\mathcal{E}_{\eta_{\varepsilon}}^{g \mathrm{gv}}$ on a trial function of the form $c_{\varepsilon} g_{\mathrm{gv}}$, where $c_{\varepsilon}$ ensures the normalization in $L^{2}\left(\mathcal{A}_{\eta_{\varepsilon}}\right)$, to get

$$
\mathcal{E}_{\eta_{\varepsilon}}^{\mathrm{gv}}\left[c_{\varepsilon} g_{\mathrm{gv}}\right] \leq c_{\varepsilon}^{4} \mathcal{E}^{\mathrm{gv}}\left[g_{\mathrm{gv}}\right]=c_{\varepsilon}^{4} E^{\mathrm{gv}},
$$

since $c_{\varepsilon} \geq 1$ and the energy is positive so that we can extend the integration domain from $\mathcal{A}_{\eta_{\varepsilon}}$ to the whole of $\mathbb{R}^{2}$. It remains then to estimate the normalization constant, but the exponential decay (3.24) together with the conditions (3.36) on $\eta_{\varepsilon}$ guarantees that $c_{\varepsilon}=1+\mathcal{O}\left(\varepsilon^{\infty}\right)$.

In the next proposition we prove the analogue of (3.24), i.e., an estimate of the decaying rate of $g_{\eta_{\varepsilon}}$. Actually we also state more refined pointwise estimates of $g_{\eta_{\varepsilon}}$ which will be crucial in the proof of the absence of vortices.

Proposition 3.5 (Pointwise estimates for $g_{\eta_{\varepsilon}}$ ). If $\Omega=\Omega_{0} \varepsilon^{-4}$ with $\Omega_{0}>0$, there exists constants $C, c>0$, such that for any $x$ with $|1-x| \geq \varepsilon^{2} \eta_{\varepsilon}^{1 / 4}$,

$$
g_{\eta_{\varepsilon}}^{2}(x) \leq C \varepsilon^{-2} \exp \left\{-c \Omega\left(|1-x|-\varepsilon^{2} \eta_{\varepsilon}^{1 / 4}\right)^{2}\right\} .
$$

Moreover if in addition $\Omega_{0}>\bar{\Omega}_{0}$ with $\bar{\Omega}_{0}=\mathcal{O}(1)$ large enough but independent of $\varepsilon$, then

$$
g_{\eta_{\varepsilon}}(x)=\left(1+\mathcal{O}\left(\Omega_{0}^{-1 / 4}\right)\right)(2 \pi)^{-1 / 2} \Omega^{1 / 4} g_{\text {osc }}\left(\Omega^{1 / 2}(1-x)\right)
$$

uniformly in $\mathrm{x} \in \mathcal{A}_{\sqrt{\eta_{\varepsilon}}}$. 
Remark 3.1 (Maximum of $g_{\eta_{\varepsilon}}$ )

A straightforward consequence of (3.43) is the estimate

$$
\left\|g_{\eta_{\varepsilon}}\right\|_{\infty}^{2} \leq \frac{1}{2} \pi^{-3 / 2} \sqrt{\alpha \Omega}\left(1+c \Omega_{0}^{-1 / 4}\right)
$$

On the other hand since $g_{\eta_{\varepsilon}}^{\prime}\left(x_{\max }\right)=0$ and $g_{\eta_{\varepsilon}}^{\prime \prime}\left(x_{\max }\right) \leq 0$, the variational equation (3.40) implies the following estimate of the maximum position

$$
\left(1-x_{\max }\right)^{2} \leq 2(\alpha \Omega)^{-2}\left[\mu_{\eta_{\varepsilon}}^{\mathrm{gv}}-2 \varepsilon^{-2} g_{\eta_{\varepsilon}}^{2}\left(x_{\max }\right)\right] \leq(\alpha \Omega)^{-1}\left(1+C \Omega_{0}^{-1 / 2}\right) .
$$

In fact, (3.48) below yields a better estimate:

$$
\begin{aligned}
\frac{1}{2} \pi^{-3 / 2} \sqrt{\alpha \Omega}\left(1-C \Omega_{0}^{-1 / 4}\right)= & \left.\left(1-C \Omega_{0}^{-1 / 4}\right)\right)(2 \pi)^{-1 / 2} \Omega^{1 / 4} g_{\mathrm{osc}}^{2}(0) \leq g_{\eta_{\varepsilon}}^{2}(1) \leq \\
& g_{\eta_{\varepsilon}}^{2}\left(x_{\max }\right) \leq \frac{1}{2} \pi^{-3 / 2} \sqrt{\alpha \Omega}\left(1+C \Omega_{0}^{-1 / 4}\right) \exp \left\{-\alpha \Omega\left(1-x_{\max }\right)^{2}\right\}
\end{aligned}
$$

which implies

$$
\left|1-x_{\max }\right| \leq C \Omega_{0}^{-5 / 8} \varepsilon^{2} .
$$

Proof of Proposition 3.5. The proof of (3.42) is basically identical to the proof of (3.24). The only difference is due to the fact that $g_{\eta_{\varepsilon}}$ does not vanish at the boundary of $\mathcal{A}_{\eta_{\varepsilon}}$. This implies that any legitimate supersolution to the variational equation (3.40) must be larger than $g_{\eta_{\varepsilon}}$ at $\partial \mathcal{A}_{\eta_{\varepsilon}}$. Alternatively one can simply repeat the proof of (3.16) or (3.24), but extend $g_{\eta_{\varepsilon}}$ outside $\mathcal{A}_{\eta_{\varepsilon}}$ to a function which decays to zero. A simple way to achieve that is to construct a function $\tilde{g}_{\eta_{\varepsilon}}$ which equals $g_{\eta_{\varepsilon}}$ in $\mathcal{A}_{\eta_{\varepsilon}}$ and satisfies the inequality

$$
-\frac{1}{2} \Delta \tilde{g}_{\eta_{\varepsilon}}+\Omega^{2} U(x) \tilde{g}_{\eta_{\varepsilon}}+2 \varepsilon^{-2} \tilde{g}_{\eta_{\varepsilon}}^{3} \leq \mu_{\eta_{\varepsilon}}^{\mathrm{gv}} \tilde{g}_{\eta_{\varepsilon}}
$$

in the region $\varepsilon^{2} \eta_{\varepsilon} \leq|1-x| \leq \varepsilon^{2} \eta_{\varepsilon}^{2}$, together with Dirichlet boundary conditions $\tilde{g}_{\eta_{\varepsilon}}\left(1 \pm \varepsilon^{2} \eta_{\varepsilon}^{2}\right)=0$. Note that $\tilde{g}_{\eta_{\varepsilon}}$ is a priori only a weak subsolution because it is in general only continuous at $1 \pm \varepsilon^{2} \eta_{\varepsilon}$. However any supersolution to (3.47) in the interval $|1-x| \leq \varepsilon^{2} \eta_{\varepsilon}^{2}$ provides a pointwise upper bound to $g_{\eta_{\varepsilon}}$ in $\mathcal{A}_{\eta_{\varepsilon}}$ and, acting as in the proof of (3.24), it is not difficult to realize that the r.h.s. of (3.42) is a supersolution.

The second statement is obtained by combining the application of the maximum principle to the variational equation (3.40) far from $x_{\max }$ with elliptic estimates like (3.23) in the central region around $x_{\max }$ where $g_{\eta_{\varepsilon}}$ is large enough. A direct consequence of the decay estimate (3.42) is indeed that one can apply the analysis contained in the proof of Proposition 3.3. In particular the analogues of (3.28) (3.34) hold true, which yield as in the proof of (3.23),

$$
\left\|\sqrt{2 \pi} \Omega^{-1 / 4} g_{\eta_{\varepsilon}}(x)-g_{\text {osc }}\left(\Omega^{1 / 2}(1-x)\right)\right\|_{L^{\infty}\left(\mathcal{A}_{\eta_{\varepsilon}}\right)} \leq C \Omega_{0}^{-1 / 4} \varepsilon^{-1} .
$$

Note that (3.42) is needed in order to extend the analysis to the whole real line exactly as in the discussion after (3.28). The estimate above is already sufficient to obtain (3.43) in the region where $g_{\eta_{\varepsilon}}$ is large enough, i.e., sufficiently close to $x_{\max }$. However far from $x_{\max }$ a different approach is needed. We start by proving the following upper bound: For any $\mathbf{x} \in \mathcal{A}_{\eta_{\varepsilon}}$ such that

$$
|1-x| \geq\left(\alpha \Omega_{0}\right)^{-1} \varepsilon^{2},
$$

there exists a constant $c$ so that

$$
g_{\eta_{\varepsilon}}^{2}(x) \leq\left(1+c \Omega_{0}^{-1 / 4}\right) \frac{1}{2} \pi^{-3 / 2} \sqrt{\alpha \Omega_{0}} \exp \left\{-\alpha \Omega\left(|1-x|-2\left(\alpha \Omega_{0}\right)^{-1} \varepsilon^{2}\right)^{2}\right\} .
$$


As above we use an extension $\tilde{g}_{\eta_{\varepsilon}}$ of $g_{\eta_{\varepsilon}}$ to the interval $|1-x| \leq \varepsilon^{2} \eta_{\varepsilon}^{2}$ and restrict for simplicity the analysis to the region

$$
1-\varepsilon^{2} \eta_{\varepsilon}^{2} \leq x \leq 1-\left(\alpha \Omega_{0}\right)^{-1} \varepsilon^{2},
$$

where we consider the function

$$
f_{\text {sup }}^{2}(x):=\left(1+c \Omega_{0}^{-1 / 4}\right) \frac{1}{2} \pi^{-3 / 2} \sqrt{\alpha \Omega_{0}} \exp \left\{-\alpha \Omega\left(1-\left(\alpha \Omega_{0}\right)^{-1} \varepsilon^{2}-x\right)^{2}\right\},
$$

At the boundary of the interval (3.51) one certainly has $\tilde{g}_{\eta_{\varepsilon}} \leq f_{\text {sup }}$ thanks to (3.48). Moreover by the following estimate of the chemical potential

$$
\mu_{\eta_{\varepsilon}}^{\mathrm{gv}}=\Omega\left[\frac{\alpha}{2}+\frac{1}{\pi} \sqrt{\frac{\alpha}{2 \pi \Omega_{0}}}+\mathcal{O}\left(\Omega_{0}^{-3 / 4}\right)+\mathcal{O}\left(\Omega^{-1 / 2}\right)\right],
$$

which is a consequence of (3.22), 3.41) and (3.48), one obtains

$$
\begin{gathered}
{\left[-\frac{1}{2} \Delta+\Omega^{2} U(x)+2 \varepsilon^{-2} f_{\text {sup }}^{2}(x)-\mu_{\eta_{\varepsilon}}^{\mathrm{gv}}\right] f_{\text {sup }}(x) \geq\left[\frac{1}{2} x^{-1} \alpha \Omega\left(2 x-1+\left(\alpha \Omega_{0}\right)^{-1} \varepsilon^{2}\right)+\right.} \\
\left.\alpha \Omega_{0} \varepsilon^{-6}\left(1-\frac{1}{2}\left(\alpha \Omega_{0}\right)^{-1} \varepsilon^{2}-x\right)+2 \varepsilon^{-2} f_{\text {sup }}^{2}(x)-\mu_{\eta_{\varepsilon}}^{\mathrm{gv}}-\mathcal{O}(1)\right] f_{\mathrm{sup}}(x) \geq \\
\sqrt{\alpha \Omega_{0}} \varepsilon^{-4}\left[\sqrt{\alpha \Omega_{0}} \varepsilon^{-2}\left(1-\frac{1}{2}\left(\alpha \Omega_{0}\right)^{-1} \varepsilon^{2}-x\right)+\pi^{-3 / 2} \exp \left\{-\alpha \Omega\left(1-\left(\alpha \Omega_{0}\right)^{-1} \varepsilon^{2}-x\right)^{2}\right\}-\right. \\
\left.2^{-1 / 2} \pi^{-3 / 2}\left(1+\mathcal{O}\left(\Omega_{0}^{-1 / 4}\right)\right)\right] f_{\text {sup }}(x) .
\end{gathered}
$$

Now we estimate for any $\delta_{1}$ such that $1-\delta_{1}(\alpha \Omega)^{-1 / 2} \leq 1-\left(\alpha \Omega_{0}\right)^{-1} \varepsilon^{2}$

$$
\begin{aligned}
& \sqrt{\alpha \Omega_{0}} \varepsilon^{-2}\left(1-\left(\alpha \Omega_{0}\right)^{-1} \varepsilon^{2}-x\right)+\pi^{-3 / 2} \exp \left\{-\alpha \Omega\left(1-\left(\alpha \Omega_{0}\right)^{-1} \varepsilon^{2}-x\right)^{2}\right\} \geq \\
& \frac{1}{2}\left(\alpha \Omega_{0}\right)^{-1 / 2}+\pi^{-3 / 2}-\pi^{-3 / 2} \alpha \Omega\left(1-\left(\alpha \Omega_{0}\right)^{-1} \varepsilon^{2}-x\right)^{2} \geq\left(1-\delta_{1}^{2}\right) \pi^{-3 / 2}-C \Omega_{0}^{-1 / 2}
\end{aligned}
$$

where we have used the trivial inequality $e^{-x} \geq 1-x$. On the other hand if $x \leq 1-\delta_{1}(\alpha \Omega)^{-1 / 2}$

$$
\sqrt{\alpha \Omega_{0}} \varepsilon^{-2}\left(1-\frac{1}{2}\left(\alpha \Omega_{0}\right)^{-1} \varepsilon^{2}-x\right) \geq \delta_{1}\left(1-C \Omega_{0}^{-1 / 2}\right) .
$$

Hence for any $\delta_{1}$ such that

$$
\frac{1}{2 \pi^{3}}<\delta_{1}^{2}<1-\frac{1}{\sqrt{2}}
$$

(e.g., $\delta_{1}=\frac{1}{2}$ ) and $\Omega_{0}$ sufficiently large, the r.h.s. of (3.54) is positive, i.e., $f_{\text {sup }}$ is a supersolution to (3.47) and (3.50) follows.

We now focus on the pointwise lower bound and prove that for any $\mathbf{x} \in \mathcal{A}_{\sqrt{\eta_{\varepsilon}}}$ such that

$$
|1-x| \geq \delta_{2}(\alpha \Omega)^{-1 / 2}, \quad \text { with } \log \sqrt{2}<\delta_{2}^{2}<1,
$$

one has the lower bound

$$
g_{\eta_{\varepsilon}}^{2}(x) \geq\left(1-\mathcal{O}\left(\varepsilon^{\infty}\right)\right) \frac{1}{2} \pi^{-3 / 2} \sqrt{\alpha \Omega_{0}} \exp \left\{-\alpha \Omega(1-x)^{2}\right\} .
$$

Define, for any $\mathbf{x} \in \mathcal{A}_{\eta_{\varepsilon}}$,

$$
f_{\text {sub }}^{2}(x):= \begin{cases}\frac{1}{2} \pi^{-3 / 2} \sqrt{\alpha \Omega_{0}}\left[\exp \left\{-\alpha \Omega(1-x)^{2}\right\}-\exp \left\{-\alpha \Omega_{0} \eta_{\varepsilon}^{2}\right\}\right], & \text { for }|1-x| \geq \delta_{\varepsilon} \\ \frac{1}{2} \pi^{-3 / 2} \sqrt{\alpha \Omega_{0}}\left[\exp \left\{-\alpha \Omega \delta_{\varepsilon}^{2}\right\}-\exp \left\{-\alpha \Omega_{0} \eta_{\varepsilon}^{2}\right\}\right], & \text { otherwise }\end{cases}
$$


where $\delta_{\varepsilon}:=\delta(\alpha \Omega)^{-1 / 2}$ and $\delta=\mathcal{O}(1)$ is a suitable parameter. Clearly $f_{\text {sub }}$ vanishes at $x=1-\varepsilon^{2} \eta_{\varepsilon}$ and, using (3.53), one can show that $f_{\text {sub }}$ is a subsolution to (3.40) in $\mathcal{A}_{\eta_{\varepsilon}}$ : If $|1-x| \geq \delta_{\varepsilon}$

$$
\begin{aligned}
&-\frac{1}{2} \Delta f_{\mathrm{sub}}+\Omega^{2} U f_{\mathrm{sub}}+2 \varepsilon^{-2} f_{\mathrm{sub}}{ }^{3}-\mu_{\eta_{\varepsilon}}^{\mathrm{gv}} f_{\mathrm{sub}} \leq \\
& {\left[\frac{1}{2} \alpha \Omega+\pi^{-3 / 2} \sqrt{\alpha \Omega_{0}} \exp \left\{-\alpha \Omega(1-x)^{2}\right\}-\mu_{\eta_{\varepsilon}}^{\mathrm{gv}}+\mathcal{O}\left(\varepsilon^{-2} \eta_{\varepsilon}\right)\right] f_{\mathrm{sub}} \leq } \\
& 2^{-1 / 2} \pi^{-3 / 2} \sqrt{\alpha \Omega_{0}} \varepsilon^{-4}\left[-1+\sqrt{2} e^{-\delta^{2}}+C \Omega_{0}^{-1 / 4}\right] f_{\mathrm{sub}} \leq 0
\end{aligned}
$$

if $\delta^{2}<\log \sqrt{2}$ and $\Omega_{0}$ is large enough. On the other hand for $|1-x| \leq \delta_{\varepsilon}$

$$
\begin{array}{r}
-\frac{1}{2} \Delta f_{\text {sub }}+\Omega^{2} U f_{\text {sub }}+2 \varepsilon^{-2} f_{\text {sub }}{ }^{3}-\mu_{\eta_{\varepsilon}}^{\mathrm{gv}} f_{\text {sub }} \leq\left[\frac{1}{2} \alpha \Omega \delta^{2}+\pi^{-3 / 2} \sqrt{\alpha \Omega_{0}} e^{-\delta^{2}}-\mu_{\eta_{\varepsilon}}^{\mathrm{gv}}+\mathcal{O}\left(\varepsilon^{-2}\right)\right] f_{\text {sub }} \leq \\
\frac{1}{2} \alpha \Omega\left[\delta^{2}-1+C \Omega_{0}^{-1 / 2}\right] f_{\text {sub }} \leq 0,
\end{array}
$$

if $\delta<1$ and $\Omega_{0}$ is large enough. Hence it suffices to take any $\delta$ satisfying $\log \sqrt{2}<\delta^{2}<1$ to obtain that $f_{\text {sub }}$ is a subsolution and $g_{\eta_{\varepsilon}} \geq f_{\text {sub }}$ inside $\mathcal{A}_{\eta_{\varepsilon}}$. However since for any $\mathbf{x} \in \mathcal{A}_{\sqrt{\eta_{\varepsilon}}}$,

$$
\exp \left\{\frac{1}{2} \alpha \Omega(1-x)^{2}-\frac{1}{2} \alpha \Omega_{0} \eta_{\varepsilon}^{2}\right\} \leq \exp \left\{-\frac{1}{2} \alpha \Omega_{0} \eta_{\varepsilon}\left(\eta_{\varepsilon}-1\right)\right\}=\mathcal{O}\left(\varepsilon^{\infty}\right),
$$

thanks to (3.36) and (3.42), then (3.56) is proven.

Collecting (3.48), (3.50) and (3.56), we obtain (3.43).

Another crucial property of $g_{\eta_{\varepsilon}}$ is its approximate symmetry w.r.t. $x=1$ : The potential $U$ is symmetric w.r.t. $x=1$ up to small corrections, so one could expect that the same is true for $g_{\eta_{\varepsilon}}$. We then introduce another reference profile $g_{\text {sym }}$ which is defined as the unique positive minimizer of the one-dimensional energy functiona 16

$$
\mathcal{E}^{\text {sym }}[f]:=2 \pi \int_{-\varepsilon^{2} \eta_{\varepsilon}}^{\varepsilon^{2} \eta_{\varepsilon}} \mathrm{d} x\left\{\frac{1}{2}\left(f^{\prime}\right)^{2}+\frac{1}{2} \alpha^{2} \Omega^{2}(1-x)^{2} f^{2}+\varepsilon^{-2} f^{4}\right\},
$$

where $f \in \mathscr{D}_{\text {sym }}$ with

$$
\mathscr{D}_{\mathrm{sym}}:=\left\{f \in H^{1}\left(\left[1-\varepsilon^{2} \eta_{\varepsilon}, 1+\varepsilon^{2} \eta_{\varepsilon}\right]\right): f=f^{*}, 2 \pi \int_{-\varepsilon^{2} \eta_{\varepsilon}}^{\varepsilon^{2} \eta_{\varepsilon}} \mathrm{d} x f^{2}(x)=1\right\} .
$$

We also define

$$
E^{\mathrm{sym}}:=\inf _{f \in \mathscr{D}_{\mathrm{sym}}} E^{\mathrm{sym}}[f]=E^{\mathrm{sym}}\left[g_{\mathrm{sym}}\right] .
$$

We collect some useful properties of $g_{\mathrm{sym}}$ in the following

Proposition 3.6 (Symmetric profile $g_{\text {sym }}$ ).

The profile $g_{\mathrm{sym}}$ satisfies the variational equation

$$
-\frac{1}{2} g_{\mathrm{sym}}^{\prime \prime}+\frac{1}{2} \alpha^{2} \Omega^{2}(1-x)^{2} g_{\mathrm{sym}}+2 \varepsilon^{-2} g_{\mathrm{sym}}^{3}=\mu^{\mathrm{sym}} g_{\mathrm{sym}}
$$

with Neumann conditions at the boundary $g_{\mathrm{sym}}^{\prime}\left(1 \pm \varepsilon^{2} \eta_{\varepsilon}\right)=0$. Moreover $g_{\mathrm{sym}}$ is symmetric under inversion w.r.t. 1 , i.e.,

$$
g_{\mathrm{sym}}(x)=g_{\mathrm{sym}}(2-x),
$$

for any $x \in\left[1-\varepsilon^{2} \eta_{\varepsilon}, 1+\varepsilon^{2} \eta_{\varepsilon}\right]$, and it has a unique maximum point at $x=1$.

\footnotetext{
${ }^{16}$ Note that $\mathcal{E}^{\text {sym }}$ coincides with $\mathcal{E}^{\text {aux }}$ except for the different integration domain.
} 
Proof. Most of the results have already been proven in a slightly different setting in Prop. 3.3 so we skip their discussion. The symmetry of $g_{\text {sym }}$ can be obtained by exploiting the invariance of the variational equation under inversion or by a rearrangement argument.

The relation between $g_{\eta_{\varepsilon}}$ and $g_{\mathrm{sym}}$ is discussed in the next proposition but we first need to state an estimate of the gradient of $g_{\eta_{\varepsilon}}$. We actually state and prove two different estimates that we use for different purposes: (3.65) below yields the $L^{\infty}$-bound one should expect from the scales of the problem, whereas (3.66) yields the expected rate of exponential decay by giving a direct access to the ratio $g_{\eta_{\varepsilon}}^{\prime} g_{\eta_{\varepsilon}}^{-1}$.

Lemma 3.1 (Estimate for $g_{\eta_{\varepsilon}}^{\prime}$ ).

If $\Omega=\Omega_{0} \varepsilon^{-4}$ with $\Omega_{0}>0$, then uniformly in $\mathbf{x} \in \mathcal{A}_{\eta_{\varepsilon}}$,

$$
\begin{gathered}
\left|g_{\eta_{\varepsilon}}^{\prime}(x)\right| \leq C(\alpha \Omega)^{3 / 2} e^{-C \Omega(1-x)^{2}}, \\
\left|g_{\eta_{\varepsilon}}^{\prime}(x)\right| \leq C \Omega_{0}^{2} \eta_{\varepsilon}^{3} \varepsilon^{-2} g_{\eta_{\varepsilon}}(x) .
\end{gathered}
$$

Proof. The proof of (3.65) is identical to that of [R1, Proposition 2.2] (actually Step 3 of that proof). Starting from the $L^{\infty}$-estimate for $g_{\eta_{\varepsilon}}$ we can use the variational equation to bound $\Delta g_{\eta_{\varepsilon}}$. Combining this with the Gagliardo-Nirenberg inequality [N, Theorem 1] we obtain the desired bound.

To prove (3.66) we can assume that $x \leq x_{\max }$ so that $g_{\eta_{\varepsilon}}^{\prime} \geq 0$, since the case $x \geq x_{\max }$ is identical. By integrating the variational equation (3.40) from $1-\varepsilon^{2} \eta_{\varepsilon}$ to $x$ and using Neumann boundary conditions, we get

$$
\begin{aligned}
-\frac{1}{2} g_{\eta_{\varepsilon}}^{\prime}(x)=\int_{1-\varepsilon^{2} \eta_{\varepsilon}}^{x} \mathrm{~d} t\left\{\frac{1}{2} t^{-1} g_{\eta_{\varepsilon}}^{\prime}(t)+\left[\mu_{\eta_{\varepsilon}}^{\mathrm{gv}}-2 \varepsilon^{-2} g_{\eta_{\varepsilon}}^{2}(t)-\Omega^{2} U^{2}(t)\right] g_{\eta_{\varepsilon}}(t)\right\} \geq \\
\quad-C \Omega^{2} g_{\eta_{\varepsilon}}(x) \int_{1-\varepsilon^{2} \eta_{\varepsilon}}^{x} \mathrm{~d} t(1-t)^{2} \geq-C \Omega^{2} \varepsilon^{6} \eta_{\varepsilon}^{3} g_{\eta_{\varepsilon}}(x),
\end{aligned}
$$

where we have used the monotonicity of $g_{\eta_{\varepsilon}}$ for $x \leq x_{\max }$ and the sup estimate $g_{\eta_{\varepsilon}} \leq \frac{1}{2} \varepsilon^{2} \mu_{\eta_{\varepsilon}}^{\mathrm{gv}}$, which is the analogue of (3.13).

Proposition 3.7 (Estimate for $g_{\eta_{\varepsilon}}-g_{\mathrm{sym}}$ ).

If $\Omega=\Omega_{0} \varepsilon^{-4}$ with $\Omega_{0}>0$ large enough, one has

$$
\left|g_{\eta_{\varepsilon}}(x)-g_{\mathrm{sym}}(x)\right| \leq C \Omega^{-1 / 4}
$$

uniformly in $\mathrm{x} \in \mathcal{A}_{\eta_{\varepsilon}}$.

Proof. Several arguments of this proof can be taken over from a similar analysis in [R1, Section 2]. We thus focus on the main new features and skip some details for brevity.

It is more convenient to work with rescaled variables: We introduce

$$
\begin{gathered}
\tilde{g}_{\eta_{\varepsilon}}(y)=(\alpha \Omega)^{-1 / 4} g_{\eta_{\varepsilon}}\left((\alpha \Omega)^{1 / 2}(1-x)\right) \\
\tilde{g}_{\mathrm{sym}}(y)=(\alpha \Omega)^{-1 / 4} g_{\mathrm{sym}}\left((\alpha \Omega)^{1 / 2}(1-x)\right)
\end{gathered}
$$

that are minimizers of the functionals

$$
\begin{aligned}
\tilde{\mathcal{E}}_{\eta_{\varepsilon}}^{\mathrm{gv}}[f] & =2 \pi \int_{\tilde{\mathcal{A}}_{\eta_{\varepsilon}}} \mathrm{d} y\left\{\frac{1}{2}\left|\partial_{y} f\right|^{2}+\left(\frac{1}{2} y^{2}+\mathcal{O}\left((\alpha \Omega)^{-1 / 2}\right)\right) f^{2}+\left(\alpha \Omega_{0}\right)^{-1 / 2} f^{4}\right\}\left(1+(\alpha \Omega)^{-1 / 2} y\right), \\
\tilde{\mathcal{E}}^{\mathrm{sym}}[f] & =2 \pi \int_{\tilde{\mathcal{A}}_{\eta_{\varepsilon}}} \mathrm{d} y\left\{\frac{1}{2}\left|\partial_{y} f\right|^{2}+\frac{1}{2} y^{2} f^{2}+\left(\alpha \Omega_{0}\right)^{-1 / 2} f^{4}\right\}
\end{aligned}
$$


where the integration is on the domain

$$
\tilde{\mathcal{A}}_{\eta_{\varepsilon}}:=\left[-\eta_{\varepsilon} \alpha^{-1 / 2}, \eta_{\varepsilon} \alpha^{-1 / 2}\right] .
$$

The quantity $\mathcal{O}\left((\alpha \Omega)^{-1 / 2}\right)$ in the potential term of $\tilde{\mathcal{E}}_{\eta_{\varepsilon}}^{\mathrm{gv}}$ comes from the Taylor expansion of the original potential. This imprecise but concise form is convenient because the precise expression is not relevant. Strictly speaking we should take into account the behavior of this term at infinity (since the domain $\tilde{\mathcal{A}}_{\eta_{\varepsilon}}$ extends to the whole real line in the limit we are considering) but any unwanted increasing term is compensated by the exponential fall-off of $\tilde{g}_{\eta_{\varepsilon}}$, inherited from that of $g_{\eta_{\varepsilon}}$. We do not elaborate more on this point as very similar subtleties have been dealt with in [R1, Sections 2 and 4.1]. In the sequel we also replace $\tilde{\mathcal{A}}_{\eta_{\varepsilon}}$ by the whole real line. Again, thanks to the fall-off of the functions under consideration, this is harmless.

We have

$$
\left(-\frac{1}{2} \partial_{y}^{2}+\frac{1}{2} y^{2}+2 \ell \tilde{g}_{\mathrm{sym}}^{2}\right) \tilde{g}_{\mathrm{sym}}=\lambda^{\mathrm{sym}} \tilde{g}_{\mathrm{sym}}
$$

where we have set

$$
\ell:=\left(\alpha \Omega_{0}\right)^{-1 / 2}
$$

and $\lambda^{\text {sym }}$ is a chemical potential fixed by the normalization, satisfying

$$
\lambda^{\mathrm{sym}}=\tilde{E}^{\mathrm{sym}}+\ell \int_{\mathbb{R}} \mathrm{d} y \tilde{g}_{\mathrm{sym}}^{4} \cdot
$$

Because of (3.68), $\tilde{g}_{\text {sym }}$ is an eigenstate of the Schrödinger operator

$$
H^{\mathrm{sym}}:=-\frac{1}{2} \partial_{y}^{2}+\frac{1}{2} y^{2}+2 \ell \tilde{g}_{\mathrm{sym}}^{2}
$$

As it is also positive (being the unique positive minimizer of (3.60), it must be the ground state $\mathrm{LL}$, Corollary 11.9]. On the other hand, we have for $\tilde{g}_{\eta_{\varepsilon}}$

$$
\left(-\frac{1}{2} \partial_{y}^{2}+\frac{1}{2} y^{2}+2 \ell \tilde{g}_{\eta_{\varepsilon}}^{2}\right) \tilde{g}_{\eta_{\varepsilon}}=\lambda \tilde{g}_{\eta_{\varepsilon}}+\mathcal{O}_{L^{2}}\left((\alpha \Omega)^{-1 / 2}\right)
$$

where

$$
\lambda=\tilde{E}_{\eta_{\varepsilon}}^{\mathrm{gv}}+\ell \int_{\mathbb{R}} \mathrm{d} y \tilde{g}_{\eta_{\varepsilon}}^{4}
$$

and the symbol $\mathcal{O}_{\mathcal{H}}(d)$ denotes a function whose norm in the Banach space $\mathcal{H}$ is bounded by $d$. The remainder in (3.70) has two origins (further details on a similar problem can be found in [R1, Proof of Theorem 2.1]):

- The remainder in the Taylor expansion of the potential. As already explained, the exponential decay of $g_{\eta_{\varepsilon}}$ allows to control the errors produced by the higher order terms in the expansion.

- The fact that the energy density in $\tilde{\mathcal{E}}_{\eta_{\varepsilon}}^{\text {gv }}$ is integrated against the measure $\left(1+(\alpha \Omega)^{-1 / 2} y\right) \mathrm{d} y$. Again, most terms are dealt with using the fall-off of $\tilde{g}_{\eta_{\varepsilon}}$. Note that there is also a term of the form $\partial_{y} \tilde{g}_{\eta_{\varepsilon}}$ (coming from the replacement of the $2 \mathrm{D}$ Laplacian of a radial function by a double radial derivative). This one is controlled using Lemma 3.1

We now write

$$
h:=\tilde{g}_{\eta_{\varepsilon}}-\tilde{g}_{\mathrm{sym}}
$$

It is a simple matter to prove that

$$
\|h\|_{L^{2}(\mathbb{R})} \leq C \ell
$$

by noting that both $\tilde{g}_{\eta_{\varepsilon}}$ and $\tilde{g}_{\text {sym }}$ are close to $g_{\text {osc }}$. Similar arguments have already been used so we omit the details. The rest of the proof consist in iteratively improving (3.72). 
Taking the difference of equations (3.68) and (3.70) we see that

$$
\left(H^{\mathrm{sym}}-\lambda^{\mathrm{sym}}\right) h=\left(\lambda-\lambda^{\mathrm{sym}}\right) \tilde{g}_{\eta_{\varepsilon}}+2 \ell \tilde{g}_{\eta_{\varepsilon}}\left(\tilde{g}_{\mathrm{sym}}^{2}-\tilde{g}_{\eta_{\varepsilon}}^{2}\right)+\mathcal{O}_{L^{2}}\left((\alpha \Omega)^{-1 / 2}\right) .
$$

We have

$$
\lambda-\lambda^{\mathrm{sym}}=\tilde{E}_{\eta_{\varepsilon}}^{\mathrm{gv}}-\tilde{E}^{\mathrm{sym}}+\ell\left(\int_{\mathbb{R}} \mathrm{d} y \tilde{g}_{\eta_{\varepsilon}}^{4}-\int_{\mathbb{R}} \mathrm{d} y \tilde{g}_{\mathrm{sym}}^{4}\right)
$$

and easy energetic arguments combined with Schwarz inequality yield

$$
\left|\lambda-\lambda^{\mathrm{sym}}\right| \leq C(\alpha \Omega)^{-1 / 2}+C \ell\|h\|_{L^{2}(\mathbb{R})} .
$$

Similarly

$$
\left\|\tilde{g}_{\eta_{\varepsilon}}\left(\tilde{g}_{\mathrm{sym}}^{2}-\tilde{g}_{\eta_{\varepsilon}}^{2}\right)\right\|_{L^{2}(\mathbb{R})} \leq C \ell\|h\|_{L^{2}(\mathbb{R})} .
$$

Thus, multiplying (3.73) by $h$ and integrating, we obtain

$$
\left\langle h\left|H^{\mathrm{sym}}-\lambda^{\mathrm{sym}}\right| h\right\rangle \leq C(\alpha \Omega)^{-1 / 2}+C \ell\|h\|_{L^{2}(\mathbb{R})}^{2},
$$

which simplifies to

$$
\left\langle h\left|H^{\mathrm{sym}}-\lambda^{\mathrm{sym}}\right| h\right\rangle \leq C \max \left((\alpha \Omega)^{-1 / 2}, \ell^{3}\right)
$$

by using (3.72). We thus have, combining the above and (3.72) and recalling that $\tilde{g}_{\text {sym }}$ is the ground state of $H^{\text {sym }}$ associated with the eigenvalue $\lambda^{\text {sym }}$

$$
\tilde{g}_{\eta_{\varepsilon}}=(1+\mathcal{O}(\ell)) \tilde{g}_{\mathrm{sym}}+\mathcal{O}_{\mathcal{H}^{\mathrm{sym}}}\left(\max \left[(\alpha \Omega)^{-1 / 2}, \ell^{3}\right]\right)
$$

where $\mathcal{H}^{\text {sym }}$ is the Banach space obtained by taking the closure w.r.t. the norm induced by $H^{\text {sym }}$ (which in particular dominates the harmonic oscillator norm), i.e.,

$$
\|f\|_{\mathcal{H}^{\text {sym }}}^{2}:=\left\langle f\left|H^{\mathrm{sym}}\right| f\right\rangle,
$$

and the remainder is orthogonal to $\tilde{g}_{\text {sym }}$. Since both $\tilde{g}_{\text {sym }}$ and $\tilde{g}_{\eta_{\varepsilon}}$ are also normalized one obtains from (3.75) the identity

$$
1=(1+\mathcal{O}(\ell))^{2}+\mathcal{O}\left(\max \left[(\alpha \Omega)^{-1}, \ell^{6}\right]\right)
$$

which implies that, since $\ell \ll 1$,

$$
\mathcal{O}(\ell)=\mathcal{O}\left(\max \left[(\alpha \Omega)^{-1}, \ell^{6}\right]\right),
$$

and therefore $\ell \leq(\alpha \Omega)^{-1}$, thus proving from (3.75D) that

$$
\|h\|_{\mathcal{H}^{\text {sym }}} \leq C(\alpha \Omega)^{-1 / 2} .
$$

To obtain (3.67) it only remains

- to recall that we are dealing with 1D functions, thus the $\mathcal{H}^{\mathrm{sym}}$-norm that controls the $H^{1}$-norm also controls the $L^{\infty}$-norm;

- to scale back the variables. 


\subsection{Energy Asymptotics and Consequences}

In this section we prove the energy asymptotics in the regime $\Omega \sim \varepsilon^{-4}$ and derive some bounds on reduced energies that will lead to the proof of the absence of vortices in the next section. As usual the asymptotics is derived from upper and lower bounds to the energy, the former being quite simple to obtain (see below) but the latter requiring more work.

The starting point is an energy decoupling already used in Section 2.2 (in the context of GL and GP theories the technique originates in [LM]).

Proposition 3.8 (Reduction to $\mathcal{A}_{\eta_{\varepsilon}}$ ).

Defining the function $u \in H^{1}\left(\mathcal{A}_{\eta_{\varepsilon}}\right)$ by setting for $\mathbf{x} \in \mathcal{A}_{\eta_{\varepsilon}}$

$$
\psi^{\mathrm{GP}}(\mathbf{x})=: g_{\eta_{\varepsilon}}(x) u(\mathbf{x}) \exp \{i\lfloor\Omega\rfloor \vartheta\},
$$

the following holds

$$
E^{\mathrm{GP}} \geq E_{\eta_{\varepsilon}}^{\mathrm{gv}}+\mathcal{E}_{\eta_{\varepsilon}}[u]
$$

with $\mathbf{B}$ given by 3.1 and

$$
\mathcal{E}_{\eta_{\varepsilon}}[u]:=\int_{\mathcal{A}_{\eta_{\varepsilon}}} \mathrm{d} \mathbf{x} g_{\eta_{\varepsilon}}^{2}\left\{\frac{1}{2}|\nabla u|^{2}-\mathbf{B} \cdot(i u, \nabla u)+\varepsilon^{-2} g_{\eta_{\varepsilon}}^{2}\left(1-|u|^{2}\right)^{2}\right\} .
$$

Moreover $u$ satisfies the following equation on $\mathcal{A}_{\eta_{\varepsilon}}$ for some $\lambda \in \mathbb{R}$

$$
-\nabla\left(g_{\eta_{\varepsilon}}^{2} \nabla u\right)-i g_{\eta_{\varepsilon}}^{2} \mathbf{B} \cdot \nabla u+2 \frac{g_{\eta_{\varepsilon}}^{4}}{\varepsilon^{2}}\left(|u|^{2}-1\right) u=\lambda g_{\eta_{\varepsilon}}^{2} u
$$

Proof. We first use the positivity of the energy density to restrict the integration in the GP functional to the annulus $\mathcal{A}_{\eta_{\varepsilon}}$. A standard energy decoupling for which we refer to [CRY, Proposition 3.1] or [CPRY1, Proposition 4.3] leads to (3.80). The variational equation satisfied by $g_{\eta_{\varepsilon}}$ is used, as well as the Neumann boundary conditions which ensure that there are no boundary terms in the decoupling. The derivation of the equation (3.82) uses the same ingredients, we refer to [CRY, Lemma 4.3].

In order to conclude the proof of Theorem[1.4 it suffices to prove a lower bound to $\mathcal{E}_{\eta_{\varepsilon}}[u]$ that matches the trivial bound which is obtained by taking $u=1$, i.e., using $g_{\eta_{\varepsilon}} \exp \{i\lfloor\Omega\rfloor \vartheta\}$ as a trial function for $\mathcal{E}^{\mathrm{GP}}$. This function has of course to be extended to the whole space and normalized. Using (3.42) to bound $g_{\eta_{\varepsilon}}$ outside $\mathcal{A}_{\eta_{\varepsilon}}$ it is not difficult to estimate the remainders due to these operations and obtain

$$
E^{\mathrm{GP}} \leq E_{\eta_{\varepsilon}}^{\mathrm{gv}}+\mathcal{O}\left(\varepsilon^{\infty}\right)
$$

To prove the corresponding lower bound we have to analyze in some details the reduced functional $\mathcal{E}_{\eta_{\varepsilon}}[u]$. As is common in the context of GP theory (see, e.g., AAB, IM1, CRY, CPRY1), a crucial step is an integration by parts of the angular momentum term. For technical reasons it will be convenient to first replace $\mathbf{B}$ with

$$
\tilde{\mathbf{B}}(x):=\Omega\left(r-r^{-1}\right) \mathbf{e}_{\vartheta}
$$

i.e., replace $\lfloor\Omega\rfloor$ with $\Omega$. We will also reduce the integration domain to $\mathcal{A}_{\sqrt{\eta_{\varepsilon}}}$ where the crucial $L^{\infty}$ estimate (3.43) holds true. These considerations lead to the definition of two potential functions that are 'anti-derivatives' of $g_{\eta_{\varepsilon}}^{2} \tilde{\mathbf{B}}$ vanishing at the boundaries of $\mathcal{A}_{\sqrt{\eta_{\varepsilon}}}$ :

$$
F_{1}(x):=\Omega \int_{1-\varepsilon^{2} \sqrt{\eta_{\varepsilon}}}^{x} \mathrm{~d} t\left(t-t^{-1}\right) g_{\eta_{\varepsilon}}^{2}(t), \quad F_{2}(x):=-\Omega \int_{x}^{1+\varepsilon^{2} \sqrt{\eta_{\varepsilon}}} \mathrm{d} t\left(t-t^{-1}\right) g_{\eta_{\varepsilon}}^{2}(t) .
$$


The use of two potential functions is a novelty of the present paper: When $x$ is smaller than $x_{\max }$ we have $\nabla^{\perp} F_{1}=g_{\eta_{\varepsilon}}^{2} \tilde{\mathbf{B}}$ and will use $F_{1}$ as potential function, whereas $F_{2}$ will be used for $x \geq x_{\max }$. In a sense this means that we use a potential function that is discontinuous at $x_{\max }$.

This apparently strange strategy is actually very useful to deal with the boundary term that the integration by parts of the angular momentum term produces. With these definitions we shall have to estimate a boundary term on the circle of radius $x_{\max }$, i.e., in the middle of the bulk, where $g_{\eta_{\varepsilon}}$ is large. This is much easier than estimating the term located on a boundary of $\mathcal{A}_{\sqrt{\eta_{\varepsilon}}}$ where the density is small, but such a term would be produced by considering a single potential function as in AAB, CRY. The necessity of this approach is due to the particular situation under consideration. The density profile $g_{\eta_{\varepsilon}}$ behaves as a Gaussian and the long tails of such a function impose the use of a larger domain (which is apparent in the fact that $\mathcal{A}_{\eta_{\varepsilon}}$ is much thicker than the characteristic length of the Gaussian) than in former situations $\mathrm{AAB}, \mathrm{CRY}, \mathrm{CPRY} 1$ where the profile was of TF type. This requirement rules out the trick we used in CPRY1, Sect. 4.3, to avoid having to estimate any boundary term at all.

We now turn to the proofs of two lemmas about the potential functions we just introduced. We first estimate the difference between $F_{1}$ and $F_{2}$ at $x_{\max }$, in other words the jump of our 'discontinuous potential'. This quantity will appear in the boundary term produced by the integration by parts. The second lemma is a pointwise estimate showing that $F_{1}$ and $F_{2}$ can be controlled by the density, which is required for the treatment of the bulk term.

Lemma 3.2 (Estimate for $\left.F_{1}-F_{2}\right)$.

For any $\mathrm{x} \in \mathcal{A}_{\sqrt{\eta_{\varepsilon}}}$ and in particular for $x=x_{\max }$

$$
\left|F_{1}(x)-F_{2}(x)\right| \leq C \Omega_{0} \eta_{\varepsilon}^{3 / 2} .
$$

Proof. We compute

$$
F_{1}(x)-F_{2}(x)=\Omega \int_{1-\varepsilon^{2} \sqrt{\eta_{\varepsilon}}}^{1+\varepsilon^{2} \sqrt{\eta_{\varepsilon}}} \mathrm{d} t\left(t-t^{-1}\right) g_{\eta_{\varepsilon}}^{2}(t)
$$

and note that a Taylor expansion yields

$$
t-\frac{1}{t}=2(t-1)+\mathcal{O}\left(\varepsilon^{4} \eta_{\varepsilon}\right)
$$

on the domain we are considering. We thus have

$$
\begin{gathered}
F_{1}(x)-F_{2}(x)=2 \Omega \int_{1-\varepsilon^{2} \sqrt{\eta_{\varepsilon}}}^{1+\varepsilon^{2} \sqrt{\eta_{\varepsilon}}} \mathrm{d} t(t-1) g_{\eta_{\varepsilon}}^{2}(t)+\mathcal{O}\left(\Omega_{0} \eta_{\varepsilon}\right)= \\
2 \Omega \int_{1-\varepsilon^{2} \sqrt{\eta_{\varepsilon}}}^{1+\varepsilon^{2} \sqrt{\eta_{\varepsilon}}} \mathrm{d} t(t-1)\left(g_{\eta_{\varepsilon}}^{2}(t)-g_{\mathrm{sym}}^{2}(t)\right)+\mathcal{O}\left(\Omega_{0} \eta_{\varepsilon}\right),
\end{gathered}
$$

where we have used the $L^{2}$-normalization of $g_{\eta_{\varepsilon}}$ and the symmetry of $g_{\text {sym }}$ to obtain the first and second error terms respectively. Finally

$$
\left|\Omega \int_{1-\varepsilon^{2} \sqrt{\eta_{\varepsilon}}}^{1+\varepsilon^{2} \sqrt{\eta_{\varepsilon}}} \mathrm{d} t(t-1)\left(g_{\eta_{\varepsilon}}^{2}(t)-g_{\mathrm{sym}}^{2}(t)\right)\right| \leq C \Omega_{0} \eta_{\varepsilon}^{3 / 2}
$$

by using the crucial estimate (3.67) and the upper bound on $g_{\eta_{\varepsilon}}$ (3.44). Recalling that $\eta_{\varepsilon} \gg 1$, (3.86) is proved.

In the following statement we will denote by $\chi_{\mathcal{S}}$ the characteristic function of a set $\mathcal{S}$. 
Lemma 3.3 (Pointwise estimate for $F_{1}$ and $F_{2}$ ).

If $\Omega=\Omega_{0} \varepsilon^{-4}$ and $\Omega_{0}>\bar{\Omega}_{0}$ with $\bar{\Omega}_{0}=\mathcal{O}(1)$ large enough but independent of $\varepsilon$, then for any $\mathbf{x} \in \mathcal{A}_{\sqrt{\eta_{\varepsilon}}}$

$$
\chi_{\left[1-\varepsilon^{2} \sqrt{\eta_{\varepsilon}}, x_{\max }\right]}(x)\left|F_{1}(x)\right|+\chi_{\left[x_{\max }, 1+\varepsilon^{2} \sqrt{\eta_{\varepsilon}}\right]}(x)\left|F_{2}(x)\right| \leq \alpha^{-1}\left(1+C \Omega_{0}^{-1 / 4}\right) g_{\eta_{\varepsilon}}^{2}(x),
$$

where the constant $C>0$ does not depend on $\mathbf{x}$.

Proof. We can assume without loss of generality that $x \leq x_{\max }$ because in the opposite case the proof is essentially the same. For any $\mathrm{x} \in \mathcal{A}_{\sqrt{\eta_{\varepsilon}}}$, 3.43) yields

$$
\begin{gathered}
\left|F_{1}(x)\right| \leq \frac{1}{2} \pi^{-3 / 2} \sqrt{\alpha \Omega_{0}} \Omega\left(1+C \Omega_{0}^{-1 / 4}\right) \int_{1-\varepsilon^{2} \sqrt{\eta_{\varepsilon}}}^{x} \mathrm{~d} t\left(1-t^{2}\right) \exp \left\{-\alpha \Omega(1-t)^{2}\right\} \leq \\
\pi^{-3 / 2} \sqrt{\alpha \Omega_{0}} \Omega\left(1+C \Omega_{0}^{-1 / 4}\right) \int_{1-x}^{\infty} \mathrm{d} z z \exp \left\{-\alpha \Omega z^{2}\right\} \leq \\
\frac{1}{2} \pi^{-3 / 2} \alpha^{-1 / 2} \Omega_{0}^{1 / 2}\left(1+C \Omega_{0}^{-1 / 4}\right) \exp \left\{-\alpha \Omega(1-x)^{2}\right\} \leq \alpha^{-1}\left(1+C \Omega_{0}^{-1 / 4}\right) g_{\eta_{\varepsilon}}^{2}(x) .
\end{gathered}
$$

The two above lemmas will be combined with some information about the function $u$. Again, we distinguish the ingredients needed for estimating the boundary term from those needed for the bulk term. The latter are quite simple actually: The bulk term involves the vorticity measure

$$
\mu:=\operatorname{curl}(i u, \nabla u)=\frac{1}{2} i \operatorname{curl}\left(u \nabla u^{*}-u^{*} \nabla u\right)
$$

a quantity that is easily related to the kinetic energy density $|\nabla u|^{2}$. Note that in our setting we do not need to apply tools involving a construction of vortex balls to obtain a useful estimate.

\section{Lemma 3.4 (Bound on the vorticity measure).}

We have

$$
|\mu| \leq|\nabla u|^{2}
$$

pointwise in $\mathcal{A}_{\eta_{\varepsilon}}$.

Proof. A straightforward computation shows that

$$
\mu=-2 \operatorname{Im}\left[\partial_{1} u\left(\partial_{2} u\right)^{*}\right]
$$

where $\partial_{i}=\partial_{x_{i}}$ for $i=1,2$ and Im stands for the imaginary part. On the other hand

$$
\left|\partial_{1} u\left(\partial_{2} u\right)^{*}\right| \leq \frac{1}{2}\left(\left|\partial_{1} u\right|^{2}+\left|\partial_{2} u\right|^{2}\right)
$$

which completes the proof.

The estimate of the boundary term will rely on the fact that the degree of $u$ around the circle of radius $x_{\max }$ is in a certain sense controlled by the kinetic energy. Note that we do not know yet that $u$ does not vanish on this circle, so (3.93) below is not truly a degree estimate. Note also that, as will be clear from the proof, the estimate holds because the circle we are interested in lies in a region where $g_{\eta_{\varepsilon}}$ is large enough. This is the main motivation for the trick of choosing a potential function discontinuous along this particular circle. If the potential function was continuous there, we would have to estimate the degree on a circle were $g_{\eta_{\varepsilon}}$ is small (namely on one of the boundaries of $\mathcal{A}_{\sqrt{\eta_{\varepsilon}}}$ ). This would be much more difficult, if not impossible. 


\section{Lemma 3.5 (Circulation estimate).}

Let $R$ be a radius satisfying

$$
R=1+\mathcal{O}\left(\Omega^{-1 / 2}\right)
$$

We have, for some constant $C>0$ and any $\delta>0$,

$$
\left|\int_{\partial B_{R}} \mathrm{~d} \sigma\left(i u, \partial_{\tau} u\right)\right| \leq C\left[\left(\Omega^{-1 / 2}+\delta\right) \int_{\mathcal{A}_{\sqrt{\eta_{\varepsilon}}}} \mathrm{d} \mathbf{x} g_{\eta_{\varepsilon}}^{2}|\nabla u|^{2}+\delta^{-1}\right]
$$

Proof. We use a smooth radial cut-off function $\chi$ with support in $[\tilde{R}, R]$, for some radius

$$
\tilde{R}=R-c \Omega^{-1 / 2}
$$

with $c>0$. Obviously one can impose

$$
\begin{aligned}
\chi(R) & =1 \\
\chi(\tilde{R}) & =0 \\
|\chi| & \leq 1 \\
|\nabla \chi| & \leq c^{-1} \Omega^{1 / 2} .
\end{aligned}
$$

We then use Stokes' formula to obtain

$$
\int_{\partial B_{R}} \mathrm{~d} \sigma\left(i u, \partial_{\tau} u\right)=\int_{\partial B_{R}} \mathrm{~d} \sigma \chi\left(i u, \partial_{\tau} u\right)=-\int_{\mathbb{R}^{2}} \mathrm{~d} \mathbf{x} \nabla^{\perp} \chi \cdot(i u, \nabla u)+\int_{\mathbb{R}^{2}} \mathrm{~d} \mathbf{x} \chi \mu,
$$

where the integrals on $\mathbb{R}^{2}$ are actually reduced to the support of $\chi$. If we choose $c$ small enough in (3.94), a combination of (3.43) and (3.92) shows that

$$
g_{\eta_{\varepsilon}}^{2} \geq C \Omega^{1 / 2}
$$

in this region. Indeed, because of the assumptions (3.92) and (3.94), the region of interest is located at a distance of at most $\mathcal{O}\left(\Omega^{-1 / 2}\right)$ from 1, i.e., in the middle of the bulk (see, e.g., Remark 3.1). We thus estimate, using also (3.91) and (3.95),

$$
\begin{aligned}
&\left|\int_{\partial B_{R}} \mathrm{~d} \sigma\left(i u, \partial_{\tau} u\right)\right| \leq C \Omega^{1 / 2} \int_{\tilde{R} \leq x \leq R} \mathrm{~d} \mathbf{x}|u||\nabla u|+\int_{\tilde{R} \leq x \leq R} \mathrm{~d} \mathbf{x}|\mu| \leq \\
& C \int_{\mathcal{A}_{\sqrt{\eta_{\varepsilon}}}} \mathrm{d} \mathbf{x} g_{\eta_{\varepsilon}}^{2}|u||\nabla u|+C \Omega^{-1 / 2} \int_{\mathcal{A}_{\sqrt{\eta_{\varepsilon}}}} \mathrm{d} \mathbf{x} g_{\eta_{\varepsilon}}^{2}|\nabla u|^{2} \leq \\
& C \delta \int_{\mathcal{A}_{\sqrt{\eta_{\varepsilon}}}} \mathrm{d} \mathbf{x} g_{\eta_{\varepsilon}}^{2}|\nabla u|^{2}+C \delta^{-1} \int_{\mathcal{A}_{\sqrt{\eta_{\varepsilon}}}} \mathrm{d} \mathbf{x} g_{\eta_{\varepsilon}}^{2}|u|^{2}+C \Omega^{-1 / 2} \int_{\mathcal{A}_{\sqrt{\eta_{\varepsilon}}}} \mathrm{d} \mathbf{x} g_{\eta_{\varepsilon}}^{2}|\nabla u|^{2} .
\end{aligned}
$$

There only remains to recall that $g_{\eta_{\varepsilon}}|u|=\left|\psi^{\mathrm{GP}}\right|$ is normalized in $L^{2}\left(\mathbb{R}^{2}\right)$ to complete the proof.

We are now able to conclude the proof of the energy lower bound. A corollary of the proof below is the following useful estimate that will allow to complete the proof of the absence of vortices in the next subsection.

\section{Corollary 3.1 (Estimates for reduced energies).}

Denoting

$$
\mathcal{F}_{\eta_{\varepsilon}}[u]:=\int_{\mathcal{A}_{\eta_{\varepsilon}}} \mathrm{d} \mathbf{x} g_{\eta_{\varepsilon}}^{2}\left\{\frac{1}{2}|\nabla u|^{2}+\varepsilon^{-2} g_{\eta_{\varepsilon}}^{2}\left(1-|u|^{2}\right)^{2}\right\},
$$

one has for $\Omega_{0}$ larger than some $\bar{\Omega}_{0}=\mathcal{O}(1)$

$$
\left|\mathcal{E}_{\eta_{\varepsilon}}[u]\right|+\mathcal{F}_{\eta_{\varepsilon}}[u] \leq C \Omega_{0}^{2} \eta_{\varepsilon}^{3}
$$

for some finite constant $C<\infty$. 
Proof of Theorem 1.4 and Corollary 3.1. The starting point is the energy decoupling of Proposition 3.8 and the trivial upper bound (3.83). We now bound from below the reduced energy $\mathcal{E}_{\eta_{\varepsilon}}[u]$.

As announced we first replace $\mathbf{B}$ with $\tilde{\mathbf{B}}$. The remainder produced by this operation is estimated as follows:

$$
\begin{array}{r}
\left|\int_{\mathcal{A}_{\eta_{\varepsilon}}} \mathrm{d} \mathbf{x} g_{\eta_{\varepsilon}}^{2}(\Omega-\lfloor\Omega\rfloor) x^{-1} \mathbf{e}_{\vartheta} \cdot(i u, \nabla u)\right| \leq \frac{1}{2} C \beta \int_{\mathcal{A}_{\eta_{\varepsilon}}} \mathrm{d} \mathbf{x} g_{\eta_{\varepsilon}}^{2}|u|^{2}+\frac{1}{2} C \beta^{-1} \int_{\mathcal{A}_{\eta_{\varepsilon}}} \mathrm{d} \mathbf{x} g_{\eta_{\varepsilon}}^{2}|\nabla u|^{2} \leq \\
\frac{1}{2} C \beta^{-1} \int_{\mathcal{A}_{\eta_{\varepsilon}}} \mathrm{d} \mathbf{x} g_{\eta_{\varepsilon}}^{2}|\nabla u|^{2}+\frac{1}{2} C \beta,
\end{array}
$$

where we have used the normalization of $\psi^{\mathrm{GP}}$ and $\beta>0$ is a constant to be fixed later in the proof. We now reduce the integration domain to $\mathcal{A}_{\sqrt{\eta_{\varepsilon}}}$ at the price of a second remainder (for a lower bound we neglect the positive terms in the energy density)

$$
\begin{aligned}
\left|\int_{\mathcal{A}_{\eta_{\varepsilon}} \backslash \mathcal{A}_{\sqrt{\eta_{\varepsilon}}}} \mathrm{d} \mathbf{x} g_{\eta_{\varepsilon}}^{2} \tilde{\mathbf{B}} \cdot(i u, \nabla u)\right| & \leq \frac{1}{2} \beta \int_{\mathcal{A}_{\eta_{\varepsilon}} \backslash \mathcal{A}_{\sqrt{\eta_{\varepsilon}}}} \mathrm{d} \mathbf{x} g_{\eta_{\varepsilon}}^{2}|\tilde{\mathbf{B}}|^{2}|u|^{2} \\
+ & +\frac{1}{2} \beta^{-1} \int_{\mathcal{A}_{\eta_{\varepsilon}} \backslash \mathcal{A}_{\sqrt{\eta_{\varepsilon}}}} \mathrm{d} \mathbf{x} g_{\eta_{\varepsilon}}^{2}|\nabla u|^{2} \leq \frac{1}{2} \beta^{-1} \int_{\mathcal{A}_{\eta_{\varepsilon}} \backslash \mathcal{A}_{\sqrt{\eta_{\varepsilon}}}} \mathrm{d} \mathbf{x} g_{\eta_{\varepsilon}}^{2}|\nabla u|^{2}+\mathcal{O}\left(\varepsilon^{\infty}\right) .
\end{aligned}
$$

Here we have used the exponential smallness of $\left|\psi^{\mathrm{GP}}\right|=g_{\eta_{\varepsilon}}|u|$ outside $\mathcal{A}_{\sqrt{\eta_{\varepsilon}}}$. This requires

$$
\eta_{\varepsilon} \gg|\log \varepsilon|,
$$

which we are free to decide. After these two steps we have

$$
\begin{aligned}
& E^{\mathrm{GP}} \geq E_{\eta_{\varepsilon}}^{\mathrm{gv}}+\int_{\mathcal{A}_{\sqrt{\eta_{\varepsilon}}}} \mathrm{d} \mathbf{x} g_{\eta_{\varepsilon}}^{2}\left\{\frac{1}{2}|\nabla u|^{2}-\Omega \tilde{\mathbf{B}} \cdot(i u, \nabla u)+\varepsilon^{-2} g_{\eta_{\varepsilon}}^{2}\left(1-|u|^{2}\right)^{2}\right\} \\
& \quad+\int_{\mathcal{A}_{\eta_{\varepsilon}} \backslash \mathcal{A}_{\sqrt{\eta_{\varepsilon}}}} \mathrm{d} \mathbf{x} g_{\eta_{\varepsilon}}^{2}\left\{\frac{1}{2}|\nabla u|^{2}+\varepsilon^{-2} g_{\eta_{\varepsilon}}^{2}\left(1-|u|^{2}\right)^{2}\right\}-\frac{1}{2} C \beta^{-1} \int_{\mathcal{A}_{\eta_{\varepsilon}}} g_{\eta_{\varepsilon}}^{2}|\nabla u|^{2}-\frac{1}{2} \beta-\mathcal{O}\left(\varepsilon^{\infty}\right)
\end{aligned}
$$

We now turn to the main part of the proof, namely a lower bound to

$$
\mathcal{E}_{\sqrt{\eta_{\varepsilon}}}[u]:=\int_{\mathcal{A}_{\sqrt{\eta_{\varepsilon}}}} \mathrm{d} \mathbf{x} g_{\eta_{\varepsilon}}^{2}\left\{\frac{1}{2}|\nabla u|^{2}-\Omega \tilde{\mathbf{B}} \cdot(i u, \nabla u)+\varepsilon^{-2} g_{\eta_{\varepsilon}}^{2}\left(1-|u|^{2}\right)^{2}\right\} .
$$

As a first step we perform the integration by parts we have been alluding to. Since

$$
\begin{array}{ll}
\nabla^{\perp} F_{1}=\Omega g_{\eta_{\varepsilon}}^{2} \tilde{\mathbf{B}}, & \text { if } 1-\varepsilon^{2} \sqrt{\eta_{\varepsilon}} \leq x \leq x_{\max }, \\
\nabla^{\perp} F_{2}=\Omega g_{\eta_{\varepsilon}}^{2} \tilde{\mathbf{B}}, & \text { if } x_{\max } \leq x \leq 1+\varepsilon^{2} \sqrt{\eta_{\varepsilon}}
\end{array}
$$

we have

$$
\begin{aligned}
& \int_{\mathcal{A}_{\sqrt{\eta_{\varepsilon}}}} \mathrm{d} \mathbf{x} g_{\eta_{\varepsilon}}^{2}\left\{\frac{1}{2}|\nabla u|^{2}-\Omega \tilde{\mathbf{B}} \cdot(i u, \nabla u)\right\}= \\
& \int_{\mathcal{A}_{\sqrt{\eta_{\varepsilon}}}} \mathrm{d} \mathbf{x} \frac{1}{2} g_{\eta_{\varepsilon}}^{2}|\nabla u|^{2}+\int_{1-\varepsilon^{2} \sqrt{\eta_{\varepsilon}} \leq x \leq x_{\max }} \mathrm{d} \mathbf{x} F_{1}(x) \mu+\int_{x_{\max } \leq x \leq 1+\varepsilon^{2} \sqrt{\eta_{\varepsilon}}} \mathrm{d} \mathbf{x} F_{2}(x) \mu \\
&-\left[F_{1}\left(x_{\max }\right)-F_{2}\left(x_{\max }\right)\right] \int_{\partial \mathcal{B}_{x_{\max }}} \mathrm{d} \sigma\left(i u, \partial_{\tau} u\right),
\end{aligned}
$$


where $\mu$ is the vorticity measure defined in (3.90). We then combine Lemmas 3.3 and 3.4 to control the bulk terms produced by the integration by parts when $\Omega_{0} \geq \bar{\Omega}_{0}$, the constant appearing in Lemma 3.3 .

$$
\begin{aligned}
\int_{\mathcal{A}_{\sqrt{\eta_{\varepsilon}}}} \mathrm{d} \mathbf{x} \frac{1}{2} g_{\eta_{\varepsilon}}^{2}|\nabla u|^{2}+\int_{1-\varepsilon^{2} \sqrt{\eta_{\varepsilon}} \leq x \leq x_{\max }} \mathrm{d} \mathbf{x} F_{1}(x) \mu & \int_{x_{\max } \leq x \leq 1+\varepsilon^{2} \sqrt{\eta_{\varepsilon}}} \mathrm{d} \mathbf{x} F_{2}(x) \mu \geq \\
& \left(\frac{1}{2}-\alpha^{-1}\left(1+\mathcal{O}\left(\Omega_{0}^{-1 / 4}\right)\right)\right) \int_{\mathcal{A} \sqrt{\eta_{\varepsilon}}} \mathrm{d} \mathbf{x} g_{\eta_{\varepsilon}}^{2}|\nabla u|^{2} .
\end{aligned}
$$

For the boundary term we use Lemmas 3.2 and 3.5 to infer

$$
\begin{array}{r}
\left|F_{1}\left(x_{\max }\right)-F_{2}\left(x_{\max }\right)\right|\left|\int_{\partial \mathcal{B}_{x_{\max }}} \mathrm{d} \sigma\left(i u, \partial_{\tau} u\right)\right| \leq C \Omega_{0} \eta_{\varepsilon}^{3 / 2}\left|\int_{\partial \mathcal{B}_{x_{\max }}} \mathrm{d} \sigma\left(i u, \partial_{\tau} u\right)\right| \leq \\
C \Omega_{0} \eta_{\varepsilon}^{3 / 2} \delta^{-1}+C \Omega_{0} \eta_{\varepsilon}^{3 / 2}\left(\Omega^{-1 / 2}+\delta\right) \int_{\mathcal{A}_{\sqrt{\eta_{\varepsilon}}}} \mathrm{d} \mathbf{x} g_{\eta_{\varepsilon}}^{2}|\nabla u|^{2}
\end{array}
$$

We thus obtain

$$
\begin{aligned}
\int_{\mathcal{A}_{\sqrt{\eta_{\varepsilon}}}} \mathrm{d} \mathbf{x} g_{\eta_{\varepsilon}}^{2}\left\{\frac{1}{2}|\nabla u|^{2}-\right. & \Omega \tilde{\mathbf{B}} \cdot(i u, \nabla u)\} \geq \\
& \left(\frac{1}{2}-C \Omega_{0} \eta_{\varepsilon}^{3 / 2} \delta-\alpha^{-1}\left(1+\mathcal{O}\left(\Omega_{0}^{-1 / 4}\right)\right)\right) \int_{\mathcal{A}_{\sqrt{\eta_{\varepsilon}}}} \mathrm{d} \mathbf{x} g_{\eta_{\varepsilon}}^{2}|\nabla u|^{2}-C \Omega_{0} \eta_{\varepsilon}^{3 / 2} \delta^{-1} .
\end{aligned}
$$

We now recall that $\alpha>2$, which is obvious from its definition (1.53). It is thus sufficient to choose

$$
\delta=C \Omega_{0}^{-1} \eta_{\varepsilon}^{-3 / 2}
$$

with a sufficiently small constant $C$ to deduce from the above that, for some $C>0$,

$$
\int_{\mathcal{A}_{\sqrt{\eta_{\varepsilon}}}} \mathrm{d} \mathbf{x} g_{\eta_{\varepsilon}}^{2}\left\{\frac{1}{2}|\nabla u|^{2}-\Omega \tilde{\mathbf{B}} \cdot(i u, \nabla u)\right\} \geq C \int_{\mathcal{A}_{\sqrt{\eta_{\varepsilon}}}} \mathrm{d} \mathbf{x} g_{\eta_{\varepsilon}}^{2}|\nabla u|^{2}-\mathcal{O}\left(\Omega_{0}^{2} \eta_{\varepsilon}^{3}\right) .
$$

Going back to (3.102) we have

$$
\mathcal{E}_{\sqrt{\eta_{\varepsilon}}}[u] \geq C \int_{\mathcal{A}_{\sqrt{\eta_{\varepsilon}}}} \mathrm{d} \mathbf{x} g_{\eta_{\varepsilon}}^{2}|\nabla u|^{2}+\int_{\mathcal{A}_{\sqrt{\eta_{\varepsilon}}}} \mathrm{dx} \frac{g_{\eta_{\varepsilon}}^{4}}{\varepsilon^{2}}\left(1-|u|^{2}\right)^{2}-\mathcal{O}\left(\Omega_{0}^{2} \eta_{\varepsilon}^{3}\right) .
$$

Combining this with (3.83) and (3.100) and taking

$$
\beta=\mathcal{O}(1)
$$

large enough, we obtain for $\Omega_{0}>\bar{\Omega}_{0}$

$$
E_{\eta_{\varepsilon}}^{\mathrm{gv}}+\mathcal{O}\left(\varepsilon^{\infty}\right) \geq E^{\mathrm{GP}} \geq E_{\eta_{\varepsilon}}^{\mathrm{gv}}+C \int_{\mathcal{A}_{\eta_{\varepsilon}}} \mathrm{d} \mathbf{x} g_{\eta_{\varepsilon}}^{2}|\nabla u|^{2}+\int_{\mathcal{A}_{\eta_{\varepsilon}}} \mathrm{d} \mathbf{x} \frac{g_{\eta_{\varepsilon}}^{4}}{\varepsilon^{2}}\left(1-|u|^{2}\right)^{2}-\mathcal{O}\left(\varepsilon^{\infty}\right)-\mathcal{O}\left(\Omega_{0}^{2} \eta_{\varepsilon}^{3}\right)
$$

if again $\eta_{\varepsilon} \gg|\log \varepsilon|$. For definiteness we fix

$$
\eta_{\varepsilon}=|\log \varepsilon|^{3 / 2}
$$

in the sequel, a choice that indeed satisfies the requirements (3.36). Neglecting the positive terms in the lower bound concludes the proof of Theorem 1.4, whereas keeping them proves Corollary 3.1. 


\subsection{Transition to the Giant Vortex State}

The proof of the absence of vortices requires one more ingredient, namely an estimate on the $L^{\infty}$-norm of the gradient of $u$. It is proved in much the same way than the corresponding results in [CRY, CPRY1] and we shall be brief on the details.

Lemma 3.6 (Gradient estimate for $\mathbf{u}$ ).

For any $c>0$, let $\mathcal{A}_{c}$ be the domain defined as

$$
\mathcal{A}_{c}:=\left\{\mathbf{x}: 1-c|\log \varepsilon|^{1 / 2} \Omega^{-1 / 2} \leq x \leq 1+c|\log \varepsilon|^{1 / 2} \Omega^{-1 / 2}\right\} .
$$

If the constant $c>0$ is chosen small enough in the above, there exists a $C>0$ such that

$$
\|\nabla u\|_{L^{\infty}\left(\mathcal{A}_{c}\right)} \leq C \varepsilon^{\frac{c^{2} \alpha}{2}-2} .
$$

Proof. The proof uses the equation (3.82) and the Gagliardo-Nirenberg inequality [N] Theorem 1]

$$
\|\nabla u\|_{L^{\infty}} \leq C\left(\|u\|_{L^{\infty}}^{1 / 2}\|\Delta u\|_{L^{\infty}}^{1 / 2}+\|u\|_{L^{\infty}}\right)
$$

as in CRY, Lemma 5.1], which leads to the following bound (we omit the details)

$$
\begin{aligned}
\|\nabla u\|_{L^{\infty}(\mathcal{D})} \leq C \varepsilon^{-1} & \left(\left\|\psi^{\mathrm{GP}}\right\|_{L^{\infty}(\mathcal{D})}+\left\|\psi^{\mathrm{GP}}\right\|_{L^{\infty}(\mathcal{D})}^{2}\left\|g_{\eta_{\varepsilon}}^{-1}\right\|_{L^{\infty}(\mathcal{D})}\right) \\
& +C\left\|\psi^{\mathrm{GP}}\right\|_{L^{\infty}(\mathcal{D})}\left\|g_{\eta_{\varepsilon}}^{-1}\right\|_{L^{\infty}(\mathcal{D})}\left(|\lambda|^{1 / 2}+\left\|g_{\eta_{\varepsilon}}^{-1} \nabla g_{\eta_{\varepsilon}}\right\|_{L^{\infty}(\mathcal{D})}+\|\mathbf{B}\|_{L^{\infty}(\mathcal{D})}\right)
\end{aligned}
$$

for any domain $\mathcal{D} \subset \mathcal{A}_{\eta_{\varepsilon}}$. We will reduce to the domain 3.105 and bound the terms on the right-hand side of the above equation. The most stringent requirement is the validity of a proper upper bound on $g_{\eta_{\varepsilon}}^{-1}$, that requires a lower bound for $g_{\eta_{\varepsilon}}$. Using (3.43) one can see that we have

$$
g_{\eta_{\varepsilon}} \geq C \varepsilon^{\frac{c^{2} \alpha}{2}-1}
$$

on $\mathcal{A}_{c}$ provided $c$ is small enough. Note that with our choice (3.104), i.e., $\eta_{\varepsilon}=|\log \varepsilon|^{3 / 2}, \mathcal{A}_{c} \subset \mathcal{A}_{\sqrt{\eta_{\varepsilon}}}$, so one can use (3.43) on $\mathcal{A}_{c}$. For the sup estimate on $\psi^{\mathrm{GP}}$ we use (3.6), whereas Lemma 3.1 yields

$$
\left\|g_{\eta_{\varepsilon}}^{-1} \nabla g_{\eta_{\varepsilon}}\right\|_{L^{\infty}\left(\mathcal{A}_{c}\right)} \leq C \Omega_{0}^{2} \varepsilon^{-2}|\log \varepsilon|^{3} .
$$

On $\mathcal{A}_{c}$ the vector potential $\mathbf{B}$ is easily seen to satisfy

$$
\|\mathbf{B}\|_{L^{\infty}\left(\mathcal{A}_{c}\right)} \leq C \Omega_{0} \varepsilon^{-2}|\log \varepsilon| .
$$

Finally, adapting the proof of [CRY, Equation (4.28)], we find

$$
|\lambda| \leq\left|\mathcal{E}_{\eta_{\varepsilon}}[u]\right|+C \varepsilon^{-3} \mathcal{F}_{\eta_{\varepsilon}}[u]^{1 / 2} \leq C \Omega_{0} \eta_{\varepsilon}^{3 / 2} \varepsilon^{-3},
$$

where the second inequality is a consequence of Corollary 3.1 and thus uses the assumption that $\Omega_{0}$ is large enough. Plugging these bounds in (3.107) we obtain the result.

We emphasize that on the domain $\mathcal{A}_{c}$ defined in 3.105 in the Lemma we have the estimate

$$
g_{\eta_{\varepsilon}} \geq C \varepsilon^{\frac{c^{2} \alpha}{2}-1} .
$$

We are now able to conclude the proof of the absence of vortices. The idea that we use, namely the combination of bounds like those in Corollary 3.1 and a gradient estimate, was first introduced in $[\mathrm{BBH}]$. We recall the definition of $\mathcal{A}_{\text {bulk }}$ :

$$
\mathcal{A}_{\text {bulk }}=\left\{\mathbf{x} \in \mathbb{R}^{2}: 1-c|\log \varepsilon|^{1 / 2} \Omega^{-1 / 2} \leq x \leq 1+c|\log \varepsilon|^{1 / 2} \Omega^{-1 / 2}\right\}, \quad c<\left(\frac{2}{\alpha}\right)^{1 / 2} .
$$


Proof of Theorem 1.3. The argument is by contradiction. Let us assume that $|1-| u(\mathbf{x})|| \geq|\log \varepsilon|^{-a}$ at some point $\mathrm{x} \in \mathcal{A}_{\mathrm{bulk}}$ for some power $a>0$. Then (3.106) means that

$$
|1-| u|| \geq \frac{1}{2}|\log \varepsilon|^{-a}, \quad \text { on } \mathcal{B}\left(\mathbf{x}, C \varepsilon^{2-\frac{c^{2} \alpha}{2}}|\log \varepsilon|^{-a}\right) \cap \mathcal{A}_{\text {bulk. }} .
$$

This implies

$$
\int_{\mathcal{A}_{\sqrt{\eta \varepsilon}}} \mathrm{d} \mathbf{x} \frac{g_{\eta_{\varepsilon}}^{4}}{\varepsilon^{2}}\left(1-|u|^{2}\right)^{2} \geq C \varepsilon^{c^{2} \alpha-2}|\log \varepsilon|^{-4 a} .
$$

This is a contradiction with (3.98) because of the choices

$$
\eta_{\varepsilon}=|\log \varepsilon|^{3 / 2}, \quad c<\left(\frac{2}{\alpha}\right)^{1 / 2} .
$$

Indeed, we would conclude that a negative power of $\varepsilon$ is controlled by a power of $|\log \varepsilon|$. We thus obtain $|1-| u|| \leq|\log \varepsilon|^{-a}$ in $\mathcal{A}_{\text {bulk }}$ for any power $a>0$, and Theorem 1.3 is proved.

The estimate of the degree of $\psi^{\mathrm{GP}}$ is a consequence of Lemma 3.5 and of the energy estimates of Corollary 3.1 .

Proof of Theorem 1.5. Taking a radius $R$ satisfying (1.58) we first note that the pointwise estimate in (1.56) implies that $\psi^{\mathrm{GP}}$ does not vanish on $\partial B_{R}$, so that its degree is indeed well defined. We then compute

$$
\begin{aligned}
2 \pi \operatorname{deg}\left(\psi^{\mathrm{GP}}, \partial B_{R}\right) & =-i \int_{\partial B_{R}} \mathrm{~d} \sigma \frac{\left|\psi^{\mathrm{GP}}\right|}{\psi^{\mathrm{GP}}} \partial_{\tau}\left(\frac{\psi^{\mathrm{GP}}}{\left|\psi^{\mathrm{GP}}\right|}\right)=-i \int_{\partial B_{R}} \mathrm{~d} \sigma \frac{|u|}{u} \partial_{\tau}\left(\frac{u}{|u|} e^{i\lfloor\Omega\rfloor \vartheta}\right) e^{-i\lfloor\Omega\rfloor \vartheta} \\
& =2 \pi\lfloor\Omega\rfloor-i \int_{\partial B_{R}} \mathrm{~d} \sigma \frac{|u|}{u} \partial_{\tau}\left(\frac{u}{|u|}\right)
\end{aligned}
$$

the second term on the second line being the degree of $u$. Then

$$
\left|\int_{\partial B_{R}} \mathrm{~d} \sigma \frac{|u|}{u} \partial_{\tau}\left(\frac{u}{|u|}\right)\right| \leq\left|\int_{\partial B_{R}} \mathrm{~d} \sigma\left(i u, \partial_{\tau} u\right)\right|
$$

where we have used that $|u|$ is bounded above and below by a constant on $\partial B_{R}$. It remains to combine (3.93) and (3.98) (where we stick to the choice (3.104) $\eta_{\varepsilon}=|\log \varepsilon|^{3 / 2}$ for definiteness and optimize over $\delta)$ and the result is proved.

\section{Rotational Symmetry Breaking}

We complete in this section the proof of Theorem 1.6. The strategy of the proof is identical to one followed in [CPRY1, Section 5], so we often omit technical details.

A symmetric vortex minimizer is a function of the form $f_{n}(x) \exp \{i n \vartheta\}$ where $f_{n}$ is real and $n \in \mathbb{Z}$. For any given $n$ there exists a minimizing function $f_{n}$ with energy $E_{n}$ but we are here interested in a global minimizer, so we denote by $\bar{n}$ a minimizer of $E_{n}$ w.r.t. $n \in \mathbb{Z}$. The associated minimizing profile $f_{\bar{n}}(x)$ is radial and positive far from the origin. Moreover it has a unique maximum at $x_{\max }$ and is increasing for $0 \leq x \leq x_{\max }$ and decreasing everywhere else.

As in [CPRY1, Proof of Theorem 1.6] the result is proven by a direct inspection of the second variation of the GP functional around a local minimizer, which in the case of a symmetric vortex $f_{n}(x) \exp \{i n \vartheta\}$ 
becomes

$$
\begin{aligned}
& \mathcal{Q}_{n}[\Xi]:=\int_{\mathbb{R}^{2}} \mathrm{~d} \mathbf{x}\left\{\frac{1}{2}\left|\left(\nabla-i \mathbf{A}_{\Omega}\right) \Xi\right|^{2}+\gamma \Omega^{2} W|\Xi|^{2}+4 \varepsilon^{-2} f_{n}^{2}|\Xi|^{2}-\mu_{n}|\Xi|^{2}\right\}+ \\
& 2 \varepsilon^{-2} \Re \int_{\mathbb{R}^{2}} \mathrm{~d} \mathbf{x} f_{n}^{2} \Xi^{2} \exp \{-2 i n \vartheta\},
\end{aligned}
$$

where $\Xi \in H^{1}\left(\mathbb{R}^{2}\right)$ and $\mu_{n}$ is the chemical potential associated with $f_{n}$, i.e., $\mu_{n}=E_{n}+\varepsilon^{-2}\left\|f_{n}\right\|_{4}^{4}$.

Proof of Theorem 1.6. We assume that the symmetric vortex with degree $\bar{n}$ is a global minimizer of the GP functional $\mathcal{E}_{\Omega}^{\mathrm{GP}}$ and then show that this yields a contradiction since one can find a function $\Xi$ which makes the quadratic form $\mathcal{Q}_{\bar{n}}$ negative.

However we start by certain useful properties of $f_{\bar{n}}$. Since we have assume that $\psi^{\mathrm{GP}}=f_{\bar{n}}(x) \exp \{i \bar{n} \vartheta\}$, the whole analysis contained in Section 3.1 applies to $f_{\bar{n}}$ too and a simple inspection shows that it can be extended to any $\Omega \gtrsim \varepsilon^{-4}$. In particular one can prove the estimates

$$
E^{\mathrm{GP}} \leq \mathcal{O}(\Omega), \quad\left\|f_{\bar{n}}\right\|_{\infty}^{2} \leq \mathcal{O}\left(\varepsilon^{2} \Omega\right), \quad\left|f_{\bar{n}}(x)\right|^{2} \leq C \varepsilon^{-2} \exp \left\{-\Omega^{1 / 2}|1-x|\right\}
$$

for any $\mathbf{x} \in \mathbb{R}^{2}$, which imply that $f_{\bar{n}}$ is concentrated in the annulus

$$
\left.\mathcal{A}_{\bar{n}}:=\left\{\mathbf{x} \in \mathbb{R}^{2}: 1-\Omega^{-1 / 2}|\log \varepsilon|^{2} \leq x \leq 1+\Omega^{1 / 2}|\log \varepsilon|^{2}\right]\right\},
$$

i.e., $\left\|f_{\bar{n}}\right\|_{L^{2}\left(\mathcal{A}_{\bar{n}}\right)}=1-o(1)$. The energy estimates (3.41) and (3.83) can as well be extended to angular velocities much larger than $\varepsilon^{-4}$, implying

$$
\mathcal{O}\left(\varepsilon^{\infty}\right) \geq \frac{1}{2} \int_{\mathcal{A}_{\bar{n}}} \mathrm{~d} \mathbf{x}\left(\bar{n} x^{-1}-\Omega x\right)^{2} f_{\bar{n}}^{2} \geq(1-o(1))\left(\bar{n}-\Omega+\mathcal{O}\left(\Omega^{1 / 2}|\log \varepsilon|^{2}\right)\right)^{2},
$$

which yields $\bar{n}=\Omega(1+o(1))$.

Now we can introduce the test function which coincides with the one used in CPRY1, Proof of Theorem 1.6], i.e.,

$$
\begin{gathered}
\Xi(\mathbf{x}):=(A(x)+B(x)) e^{i(\bar{n}+d) \vartheta}+(A(x)-B(x)) e^{i(\bar{n}-d) \vartheta}, \\
A(x):=\left\{\begin{array}{ll}
x^{d+1} f_{\bar{n}}^{\prime}, & \text { for } 0 \leq x \leq x_{\max }, \\
0, & \text { otherwise, }
\end{array} \quad B(x):= \begin{cases}\bar{n} x^{d} f_{\bar{n}}, & \text { for } 0 \leq x \leq x_{\max }, \\
\bar{n} x_{\max }^{d} f_{\bar{n}}, & \text { otherwise. }\end{cases} \right.
\end{gathered}
$$

A somewhat lengthy computation yields

$$
\begin{array}{r}
\mathcal{Q}_{\bar{n}}[\Xi]=4 \pi \int_{0}^{x_{\max }} \mathrm{d} x x^{2 d+2} f_{\bar{n}} f_{\bar{n}}^{\prime}\left\{2(d+1) \mu_{\bar{n}}+2 \Omega \bar{n}-(d+2) \Omega^{2} x^{2}-2 \gamma(d+1) \Omega^{2} W(x)+\right. \\
\left.\gamma \Omega^{2} x W^{\prime}(x)-4(d+1) \varepsilon^{-2} f_{\bar{n}}^{2}\right\}+2 \pi \bar{n}^{2} d^{2} x_{\max }^{2 d} \int_{x_{\max }}^{\infty} \mathrm{d} x x^{-1} f_{\bar{n}}^{2} .
\end{array}
$$

Now using the facts

$$
\bar{n}=\Omega(1+o(1)), \quad x_{\max }=1+o(1), \quad W(x)=o(1), \quad W^{\prime}(x)=o(1),
$$

for any $\mathrm{x} \in \mathcal{A}_{\bar{n}}$, as well as the monotonicity of $f_{\bar{n}}$ for $x \leq x_{\max }$, we obtain

$$
\begin{aligned}
\mathcal{Q}_{\bar{n}}[\Xi] \leq-4 \pi \Omega^{2} d(1-o(1)) \int_{0}^{x_{\max }} \mathrm{d} x x^{2 d+2} f_{\bar{n}} f_{\bar{n}}^{\prime}+C \Omega^{2} d^{2} \leq \\
-2 \pi \Omega^{2} d\left[(1-o(1)) f_{\bar{n}}^{2}\left(x_{\max }\right)-C d\right]<0
\end{aligned}
$$

for $\varepsilon$ sufficiently small and any finite $d>0$ since $f_{\bar{n}}^{2}\left(x_{\max }\right)=\mathcal{O}\left(\varepsilon^{2} \Omega\right) \gg 1$. 
Acknowledgements. MC and NR acknowledge the hospitality of the Erwin Schrödinger Institute for Mathematical Physics in Vienna where part of this work was carried out. The work of NR was supported by the European Research Council under the European Community Seventh Framework Programme (FP7/2007-2013 Grant Agreement MNIQS no. 258023), the work of MC by the same programme under the Grant Agreement CoMBoS no. 239694.

\section{References}

[A] A. Aftalion, Vortices in Bose-Einstein Condensates, Progress in Nonlinear Differential Equations and their Applications 67, Birkhäuser, Basel, 2006.

[AB] A. Aftalion, X. Blanc, Reduced Energy Functionals for a Three Dimensional Fast Rotating Bose-Einstein Condensate, Ann. Inst. H. Poincaré C: Anal. Non Linéaire 25 (2008), 339-355.

[ABN] A. Aftalion, X. Blanc, F. Nier, Lowest Landau Level Functionals and Bargmann Spaces for Bose-Einstein Condensates, J. Funct. Anal. 241 (2006), 661-702.

[AJR] A. Aftalion, R. L. Jerrard, J. Royo-Letelier, Non Existence of Vortices in the Small Density Region of a Condensate, J. Funct. Anal. 260 (2011), 2387-2406.

[AAB] A. Aftalion, S. Alama, L. Bronsard, Giant Vortex and the Breakdown of Strong Pinning in a Rotating Bose-Einstein Condensate, Arch. Rational Mech. Anal. 178 (2005), 247-286.

[BBH] F. BÉthuel, H. Brézis, F. HÉLein, Asymptotics for the Minimization of a Ginzburg-Landau Functional, Calc. Var. Partial Differential Equations 1 (1993), 123-148.

[CD] Y. Castin, R. Dum, Bose-Einstein Condensates with Vortices in Rotating Traps, Eur. Phys. J. D 7 (1999), 399-412.

[BCPY] J.-B. Bru, M. Correggi, P. Pickl, J. Yngvason, The TF Limit for Rapidly Rotating Bose Gases in Anharmonic Traps, Comm. Math. Phys. 280 (2008), 517-544.

[CDY1] M. Correggi, T. Rindler-Daller, J. Yngvason, Rapidly Rotating Bose-Einstein Condensates in Strongly Anharmonic Traps, J. Math. Phys. 48 (2007), 042104.

[CDY2] M. Correggi, T. Rindler-Daller, J. Yngvason, Rapidly Rotating Bose-Einstein Condensates in Homogeneous Traps, J. Math. Phys. 48 (2007), 102103.

[CPrY1] M. Correggi, F. Pinsker, N. Rougerie, J. Yngvason, Critical Rotational Speeds in the Gross-Pitaevskii Theory on a Disc with Dirichlet Boundary Conditions, J. Stat. Phys. 143 (2011), 261-305.

[CPrY2] M. Correggi, F. Pinsker, N. Rougerie, J. Yngvason, Rotating Superfluids in Anharmonic Traps: From Vortex Lattices to Giant Vortices, Phys. Rev. A 84 (2011), 053614.

[CRY] M. Correggi, N. Rougerie, J. Yngvason, The Transition to a Giant Vortex Phase in a Fast Rotating Bose-Einstein Condensate, Commun. Math. Phys. 303 (2011), 451-508.

[CY] M. Correggi, J. Yngvason, Energy and Vorticity in Fast Rotating Bose-Einstein Condensates, J. Phys. A: Math. Theor. 41 (2008), 445002.

[E] L.C. Evans, Partial Differential Equation, Graduate Studies in Mathematics 19, AMS, Providence, 1998. 
[ESY1] L. Erdős, B. Schlein, H.T. YAu, Rigorous Derivation of the Gross-Pitaevskii Equation, Phys. Rev. Lett. 98 (2007), 040404.

[ESY2] L. Erdős, B. Schlein, H.T. YAu, Derivation of the Gross-Pitaevskii Equation for the Dynamics of Bose-Einstein Condensate, Ann. Math. 172 (2010), 291-370.

[Fe1] A.L. Fetter, Rotating Trapped Bose-Einstein Condensates, Rev. Mod. Phys. 81 (2009), 647691.

[Fe2] A.L. Fetter, Rotating Vortex Lattice in a Bose-Einstein Condensate Trapped in Combined Quadratic and Quartic Radial Potentials, Phy. Rev. A 64 (2001), 063608.

[FJS] A.L. Fetter, N. Jackson, S. Stringari, Rapid Rotation of a Bose-Einstein Condensate in a Harmonic Plus Quartic Trap, Phys. Rev. A 71 (2005), 013605.

[FB] U.R. Fischer, G. BAym, Vortex States of Rapidly Rotating Dilute Bose-Einstein Condensates, Phys. Rev. Lett. 90 (2003), 140402.

[FZ] H. Fu, E. Zaremba, Transition to the Giant Vortex State in a Harmonic-plus-quartic Trap, Phys. Rev. A 73 (2006), 013614.

[IM1] R. Ignat, V. Millot, The Critical Velocity for Vortex Existence in a Two-dimensional Rotating Bose-Einstein Condensate, J. Funct. Anal. 233 (2006), 260-306.

[IM2] R. Ignat, V. Millot, Energy Expansion and Vortex Location for a Two Dimensional Rotating Bose-Einstein Condensate, Rev. Math. Phys. 18 (2006), 119-162.

[JS] R.L. Jerrard, H.M. Soner, The Jacobian and the Ginzburg-Landau Energy, Calc. Var. Partial Differential Equations 14 (2002), 524-561.

[KTU] K. Kasamatsu, M. Tsubota, M. Ueda, Giant Hole and Circular Superflow in a Fast Rotating Bose-Einstein Condensate, Phys. Rev. A 66 (2002), 050606.

[KB] G.M. Kavoulakis, G. Baym, Rapidly Rotating Bose-Einstein Condensates in Anharmonic Potentials, New J. Phys. 5 (2003), 51.1-51.11.

[KF] J.K. Kim, A.L. Fetter, Dynamics of a Rapidly Rotating Bose-Einstein Condensate in a Harmonic Plus Quartic trap, Phys. Rev. A 72 (2005), 023619.

[LM] L. Lassoued, P. Mironescu, Ginzburg-Landau Type Energy with Discontinuous Constraint, J. Anal. Math. 77 (1999), 1-26.

[LeS] M. Lewin, R. Seiringer, Strongly Correlated Phases in Rapidly Rotating Bose Gases, J. Stat. Phys. 137 (2009), 1040-1062.

[LL] E.H. Lieb, M. Loss, Analysis, Graduate Studies in Mathematics 14, AMS, Providence, 1997.

[LiS] E.H. Lieb, R. SEIRInger, Derivation of the Gross-Pitaevskii Equation for Rotating Bose Gases, Comm. Math. Phys. 264 (2006), 505-537.

[LSSY] E.H. Lieb, R. Seiringer, J.P. Solovej, J. Yngvason, The Mathematics of the Bose Gas and its Condensation, Oberwolfach Seminar Series 34, Birkhäuser, Basel (2005).

[LSY1] E.H. Lieb, R. Seiringer, J. Yngvason, Bosons in a Trap: A Rigorous Derivation of the Gross-Pitaevskii Energy Functional, Phys. Rev. A. 61 (2000), 043602. 
[LSY2] E.H. Lieb, R. Seiringer, J. Yngvason, The Yrast Line of a Rapidly Rotating Bose Gas: The Gross-Pitaevskii Regime, Phys. Rev. A 79 (2009), 063626.

[N] L. Nirenberg, An extended interpolation inequality, Ann. Scuola Norm. Sup. Pisa 20 (1966), $733-737$.

[P] P. Pickl, Derivation of the Time Dependent Gross Pitaevskii Equation with External Fields, preprint arXiv:1001.4894v2 [math-ph] (2010).

[R1] N. Rougerie, The Giant Vortex State for a Bose-Einstein Condensate in a Rotating Anharmonic Trap: Extreme Rotation Regimes, J. Math. Pures Appl. 95 (2011), 296-347.

[R2] N. Rougerie, Vortex Rings in Fast Rotating Bose-Einstein Condensates, Archive of Rational Mechanics and Analysis 203 (2012), 69.

[SS1] E. Sandier, S. Serfaty, On the Energy of Type-II Superconductors in the Mixed Phase, Rev. Math. Phys. 12 (2000), 1219-1257.

[SS2] E. Sandier, S. Serfaty, Vortices in the Magnetic Ginzburg-Landau Model, Progress in Nonlinear Differential Equations and their Applications 70, Birkhäuser, Basel, 2007, erratum available at http://www.ann.jussieu.fr/serfaty/publis.html.

[SS3] E. Sandier, S. Serfaty, From the Ginzburg-Landau Model to Vortex Lattice Problems, preprint arXiv:1011.4617v2 [math.AP] (2010).

[Seir] R. Seiringer, Gross-Pitaevskii Theory of the Rotating Bose Gas, Commun. Math. Phys. 229 (2002), 491-509.

[T] M.E. TAYLOR, Partial Differential Equations: Basic Theory, Springer-Verlag, New York, 1996. 